\title{
Mechanical properties and microstructure characterization of unirradiated Eurofer-97 steel variants for the EUROfusion project
}

Approved for public release. Distribution is unlimited.
A. Bhattacharya
X. Chen
K. Linton
Y. Yamamoto
M. Sokolov
L. Clowers
Y. Katoh

June 17, 2018 


\title{
DOCUMENT AVAILABILITY
}

Reports produced after January 1, 1996, are generally available free via US Department of Energy (DOE) SciTech Connect.

Website http://www.osti.gov/scitech/

Reports produced before January 1, 1996, may be purchased by members of the public from the following source:

\author{
National Technical Information Service \\ 5285 Port Royal Road \\ Springfield, VA 22161 \\ Telephone 703-605-6000 (1-800-553-6847) \\ TDD 703-487-4639 \\ Fax 703-605-6900 \\ E-mail info@ntis.gov \\ Website http://classic.ntis.gov/
}

Reports are available to DOE employees, DOE contractors, Energy Technology Data Exchange representatives, and International Nuclear Information System representatives from the following source:

Office of Scientific and Technical Information

PO Box 62

Oak Ridge, TN 37831

Telephone 865-576-8401

Fax 865-576-5728

E-mail reports@osti.gov

Website http://www.osti.gov/contact.html

This report was prepared as an account of work sponsored by an
agency of the United States Government. Neither the United States
Government nor any agency thereof, nor any of their employees, makes
any warranty, express or implied, or assumes any legal liability or
responsibility for the accuracy, completeness, or usefulness of any
information, apparatus, product, or process disclosed, or represents that
its use would not infringe privately owned rights. Reference herein to
any specific commercial product, process, or service by trade name,
trademark, manufacturer, or otherwise, does not necessarily constitute
or imply its endorsement, recommendation, or favoring by the United
States Government or any agency thereof. The views and opinions of
authors expressed herein do not necessarily state or reflect those of the
United States Government or any agency thereof.


Materials Science and Technology Division

\title{
Mechanical properties and microstructure characterization of unirradiated Eurofer-97 steel variants for the EUROfusion project.
}

\author{
Arunodaya Bhattacharya \\ Xiang Chen \\ Kory Linton \\ Yukinori Yamamoto \\ Mikhail Sokolov \\ Logan Clowers \\ Yutai Katoh
}

June 2018

Prepared by

OAK RIDGE NATIONAL LABORATORY

Oak Ridge, TN 37831-6283

managed by

UT-BATTELLE, LLC

for the

Karlsruhe Institute of Technology

under contract NFE-16-06240 


\section{CONTENTS}

1. INTRODUCTION

2. MATERIALS AND METHODS

(n)

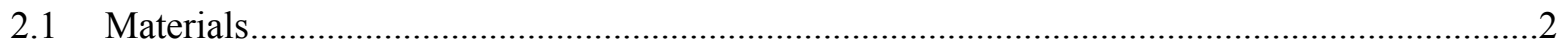

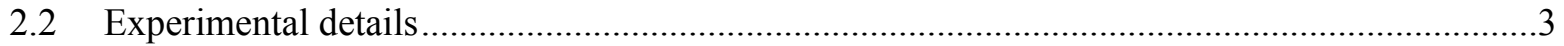

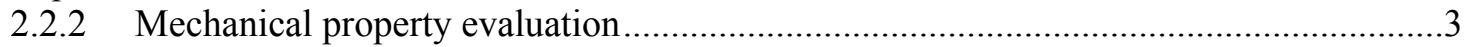

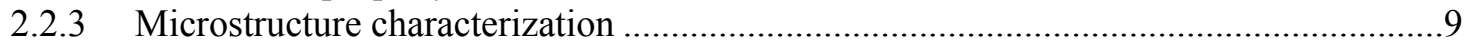

3. RESULTS

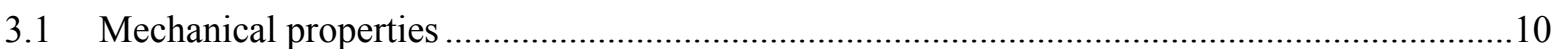

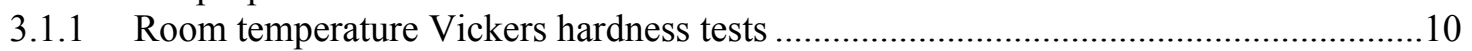

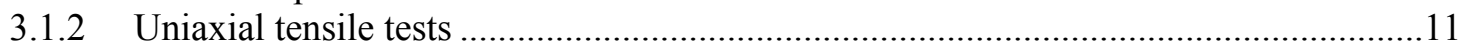

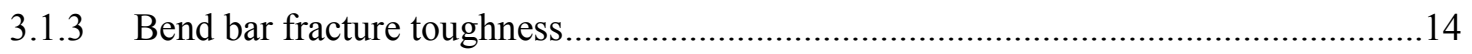

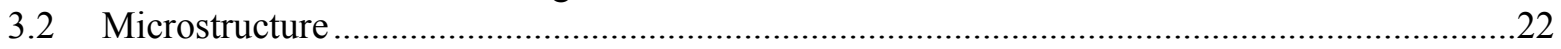

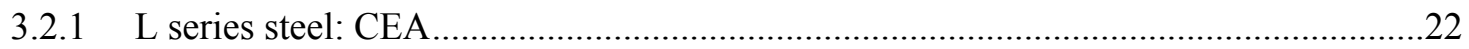

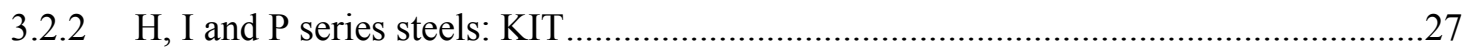

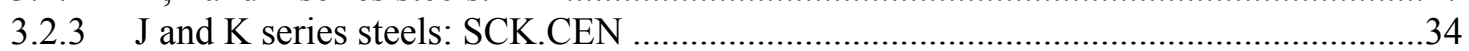

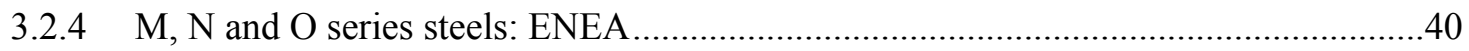

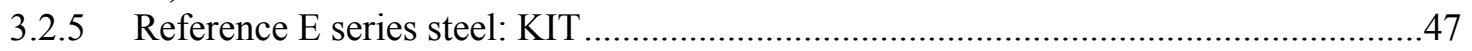

References

Appendix 



\section{INTRODUCTION}

To understand the microstructure and mechanical property degradation of various Eurofer-97 steel variants being neutron irradiated at HFIR up to 2.5 and 20 dpa for the EUROfusion programme, it is necessary to develop a general understanding of these steels in non-irradiated form. In this context, room temperature mechanical property evaluation tests and multi-length scale microstructure characterization of the different Eurofer-97 steel variants: H, I, J, K, L, M, N, O, P and reference E series steels, was performed at ORNL. Mechanical tests consisted of Vickers hardness indentation measurements, uniaxial tensile tests and fracture toughness tests. Microstructure characterization consisted of light optical microscopy (LOM), transmission electron microscopy (TEM) in both conventional TEM and scanning TEM (STEM) mode, energy dispersive X-ray spectroscopy (EDX) in STEM mode, study of the fracture surfaces after room temperature tensile tests, and general microstructure observations using SEM. High temperature mechanical tests will be performed once the thermometry results are available for the 2.5 dpa campaign.

This report summarizes the microstructure and mechanical property results of the various EUROfusion steels. LOM image, Vickers hardness and tensile test results of the old reference $\mathrm{G}$ and $\mathrm{F}$ series are also provided in the appendix 


\section{MATERIALS AND METHODS}

\subsection{Materials}

The different steels that were provided by KIT for the irradiation campaign, on which characterization was performed in unirradiated form are summarized in Table 1. These specimens were in the shape of subsized tensiles (SS-J3), 4-notch fracture toughness bend bar specimens (M4CVN) and MPC1 multipurpose coupons.

Tables 1 and 2 also summarize the heat treatment and chemical composition information that was provided to ORNL. It must be noted that after discovering out-of-spec properties for G/F series reference Eurofer-97 steel, new reference steel bar was provided to ORNL, from which samples were machined locally. This new reference is the E- series in the tables below.

Table 1. Summary of different Eurofer-97 steel variants. AQ: air quenched, WQ: water quenched, LT: low temperature application, HT: high temperature application.

\begin{tabular}{|c|c|c|c|c|}
\hline M-Code & Material type & Heat & Condition & Provider \\
\hline $\mathrm{E}$ & EUROFER97/2 & 993391 & $980^{\circ} \mathrm{C} / 0.5 \mathrm{~h}+\mathrm{AQ}+760^{\circ} \mathrm{C}+\mathrm{AC}$ (new reference) & KIT \\
\hline $\mathrm{H}$ & EUROFER-LT & $\mathrm{J} 362 \mathrm{~A}$ & $1000^{\circ} \mathrm{C} / 0.5 \mathrm{~h}+\mathrm{WQ}+820^{\circ} \mathrm{C}+\mathrm{AC}$ & KIT \\
\hline I & EUROFER-LT & J363A & $1000^{\circ} \mathrm{C} / 0.5 \mathrm{~h}+\mathrm{WQ}+820^{\circ} \mathrm{C}+\mathrm{AC}$ & KIT \\
\hline $\mathrm{P}$ & EUROFER-LT & J361A & $1000^{\circ} \mathrm{C} / 0.5 \mathrm{~h}+\mathrm{WQ}+820^{\circ} \mathrm{C}+\mathrm{AC}$ & KIT \\
\hline $\mathrm{L}$ & EUROFER97/2 & 994578 & $1150^{\circ} \mathrm{C} / 0.5 \mathrm{~h}+\mathrm{AQ}+700^{\circ} \mathrm{C}+\mathrm{AC}$ & CEA \\
\hline \multirow[t]{2}{*}{$\mathrm{J}$} & \multirow[t]{2}{*}{ EUROFER-LT } & \multirow[t]{2}{*}{ I196C } & $\begin{array}{l}\text { TMT: } 1250^{\circ} \mathrm{C} / 1 \mathrm{~h} \text { and then rolling to a final rolling } \\
\text { temperature of } 850^{\circ} \mathrm{C} \text { in } 6 \text { rolling steps with a } \\
\text { reduction of } 20-30 \% \text { for each rolling pass, then } \mathrm{AC} \text {. }\end{array}$ & \multirow[t]{2}{*}{ SCK.CEN } \\
\hline & & & Q\&T: $880^{\circ} \mathrm{C} / 0.5 \mathrm{~h}+\mathrm{WQ}+750^{\circ} \mathrm{C} / 2 \mathrm{~h}+\mathrm{AC}$ & \\
\hline \multirow[t]{2}{*}{$\mathrm{K}$} & \multirow[t]{2}{*}{ EUROFER-HT } & \multirow[t]{2}{*}{ I427A } & $\begin{array}{l}\text { TMT: } 1250^{\circ} \mathrm{C} / 1 \mathrm{~h} \text { and then rolling to a final rolling } \\
\text { temperature of } 850^{\circ} \mathrm{C} \text { in } 6 \text { rolling steps with a } \\
\text { reduction of } 20-30 \% \text { for each rolling pass, then AC. }\end{array}$ & \multirow[t]{2}{*}{ SCK.CEN } \\
\hline & & & Q\&T: $1050^{\circ} \mathrm{C} / 15 \mathrm{~min}+\mathrm{WQ}+675^{\circ} \mathrm{C} / 1.5 \mathrm{~h}+\mathrm{AC}$ & \\
\hline M & EUROFER97/2 & 993391 & $\begin{array}{c}1020^{\circ} \mathrm{C} / 0.5 \mathrm{~h}+\mathrm{AQ}+1020^{\circ} \mathrm{C} / 0.5 \mathrm{~h}+\mathrm{AQ} \\
+760^{\circ} \mathrm{C} / 1.5 \mathrm{~h}+\mathrm{AC}(\text { double austenitization })\end{array}$ & ENEA \\
\hline \multirow[t]{2}{*}{$\mathrm{O}$} & \multirow[t]{2}{*}{ EUROFER-LT } & \multirow[t]{2}{*}{ VM2991 } & $\begin{array}{l}\text { TMT: } 1080^{\circ} \mathrm{C} / 1 \mathrm{~h} \text {, cooling to } 650^{\circ} \mathrm{C} \text { and rolling, } \\
\text { reduction } 40 \% \text { (from } 30 \mathrm{~mm} \text { to } 18 \mathrm{~mm} \text { ) }\end{array}$ & \multirow[t]{2}{*}{ ENEA } \\
\hline & & & Tempering: $760^{\circ} \mathrm{C} / 1 \mathrm{~h}+\mathrm{AC}$ & \\
\hline $\mathrm{N}$ & EUROFER-LT & VM2897 & $\begin{array}{c}920^{\circ} \mathrm{C} / 1.5 \mathrm{~h}+\mathrm{AQ}+920^{\circ} \mathrm{C} / 1.5 \mathrm{~h}+\mathrm{AQ}+760^{\circ} \mathrm{C} / 1 \mathrm{~h}+ \\
\mathrm{AC} \text { (double austenitization) }\end{array}$ & ENEA \\
\hline
\end{tabular}


Table 2. Chemical compositions of different Eurofer-97 steel variants provided by KIT. All values are in wt.\%.

\begin{tabular}{|c|c|c|c|c|c|c|c|c|c|}
\hline Element & $\mathbf{P}$ & $\mathbf{H}$ & $\mathbf{I}$ & $\begin{array}{c}\mathbf{G}, \mathbf{E}, \\
\mathbf{M}\end{array}$ & $\mathbf{L}$ & $\mathbf{J}$ & $\mathbf{K}$ & $\mathbf{O}$ & $\mathbf{N}$ \\
\hline $\mathrm{Cr}$ & 8.7 & 8.7 & 8.73 & 8.83 & 9.14 & 9 & 7.84 & 8.8 & 9.04 \\
\hline $\mathrm{C}$ & 0.105 & 0.0583 & 0.11 & 0.107 & 0.106 & 0.107 & 0.017 & 0.06 & 0.092 \\
\hline $\mathrm{Mn}$ & 0.021 & 0.0223 & 0.0189 & 0.527 & 0.54 & 0.39 & $<0.03$ & 0.5 & 0.11 \\
\hline $\mathrm{V}$ & 0.2 & 0.353 & 0.351 & 0.2 & 0.2 & 0.22 & 0.2212 & 0.3 & $<0.05$ \\
\hline $\mathrm{N}$ & 0.0445 & 0.0465 & 0.0422 & 0.019 & 0.038 & 0.022 & 0.022 & 0.07 & 0.0024 \\
\hline $\mathrm{W}$ & 1.14 & 1.07 & 1.08 & 1.081 & 1.11 & 1.1 & 0.99 & 0.97 & 0.99 \\
\hline $\mathrm{Ta}$ & 0.093 & 0.1 & 0.0918 & 0.117 & 0.12 & 0.11 & 0.1265 & 0.05 & 0.092 \\
\hline $\mathrm{Si}$ & 0.032 & 0.0362 & 0.0363 & 0.0352 & 0.025 & $<0.04$ & $<0.04$ & 0.15 & 0.037 \\
\hline $\mathrm{S}$ & 0.0015 & 0.0014 & 0.0011 & 0.0009 & 0.0037 & 0.0011 & 0.0011 & 0.003 & 0.001 \\
\hline $\mathrm{B}$ & $<0.0005$ & $<0.0005$ & $<0.0005$ & $<0.0005$ & 0.0011 & $<0.0005$ & $<0.0005$ & $<0.001$ & $<0.001$ \\
\hline $\mathrm{Ti}$ & $<0.0001$ & $<0.0001$ & $<0.0001$ & $<0.0001$ & 0.001 & $<0.0001$ & $<0.0001$ & $<0.01$ & $<0.01$ \\
\hline $\mathrm{O}$ & 0.0036 & 0.0022 & 0.0052 & 0.0043 & $<0.001$ & 0.007 & 0.0043 & 0.006 & $<0.004$ \\
\hline $\mathrm{Nb}$ & $<0.0004$ & $<0.0004$ & $<0.0004$ & $<0.0004$ & 0.004 & $<0.0004$ & $<0.0004$ & $<0.01$ & $<0.01$ \\
\hline $\mathrm{Mo}$ & 0.0012 & 0.0009 & 0.0009 & 0.0009 & 0.002 & & & $<0.01$ & $<0.01$ \\
\hline $\mathrm{Ni}$ & 0.0057 & 0.004 & 0.0036 & 0.0034 & 0.01 & & & $<0.02$ & $<0.02$ \\
\hline $\mathrm{Cu}$ & 0.0075 & 0.0077 & 0.0072 & 0.007 & 0.003 & & & $<0.01$ & $<0.01$ \\
\hline $\mathrm{Al}$ & 0.0016 & 0.0017 & 0.0014 & 0.0012 & 0.002 & & & $<0.01$ & $<0.01$ \\
\hline $\mathrm{Co}$ & 0.0021 & 0.0017 & 0.0017 & 0.0017 & 0.003 & & & $<0.01$ & $<0.01$ \\
\hline $\mathrm{As}, \mathrm{Zr}, \mathrm{Sn}, \mathrm{Sb}$ & & & & & $<0.007$ & & & $<0.005$ & $<0.005$ \\
\hline $\mathrm{P}$ & & & & & 0.0015 & & & $<0.006$ & $<0.005$ \\
\hline & & & & & & & & & \\
\hline
\end{tabular}

\subsection{Experimental details}

\subsubsection{Mechanical property evaluation}

As a first step, Vickers microhardness indentation tests were performed on the non-irradiated samples in accordance with ASTM E384 Standard Test Method for Microindentation Hardness of Materials, using $1 \mathrm{~kg}$ load, $15 \mathrm{~s}$ dwell time. Among other things, this standard mainly imposes the restriction on distance between indents and the distance between an indent and sample edge: both must be minimum $2.5 \mathrm{dV}$, where $\mathrm{dV}$ represents Vickers diagonal. These tests were conducted using a Future Tech Model FM-7 microhardness testing machine on the head/grip section of SS-J3 tensile specimens. At least ten indents were measured to provide an average hardness value of each steel in the irradiation matrix (with five indents per sample). For the reference E series steel, forty-seven indents were measured using nine different SS-J3 samples.

Uniaxial tensile tests were performed at room temperature on the non-irradiated SS-J3 miniature tensile specimens per the ASTM E8 Standard Test Methods for Tension Testing of Metallic Materials, using a strain rate of $10^{-3} \mathrm{~s}^{-1}\left(5 \times 10^{-3} \mathrm{~mm} / \mathrm{s}\right.$ extension rate $)$. The specimens were shoulder loaded for testing using an MTS servo-hydraulic frame with $89-\mathrm{kN}$ capacity. Fig. 1 shows a schematic of SS-J3 specimens of 
dimensions $16 \mathrm{~mm}$ x $4 \mathrm{~mm} \times 0.76 \mathrm{~mm}$, and $5 \mathrm{~mm}$ gauge length. Due to unavailability of a contact or noncontact extensometer for such small samples, the elongation was estimated by machine stroke. Using machine stroke obviously introduces uncertainties in the elongation behaviour in the elastic regime due to machine compliance, but it does not affect the plastic regime or the calculated strength values. Because the elongation is provided by machine stroke, the strain rate is the extension rate and not the cross-head speed as is taken in cases with an extensometer. To eliminate any possible misleading elongation values arising due to machine compliance, we have also included the plastic strain information in the report and this should serve as the basis for comparing different steels. High temperature (irradiation temperature) tensile tests on the unirradiated specimens will be conducted once the thermometry data of the irradiated samples is available.

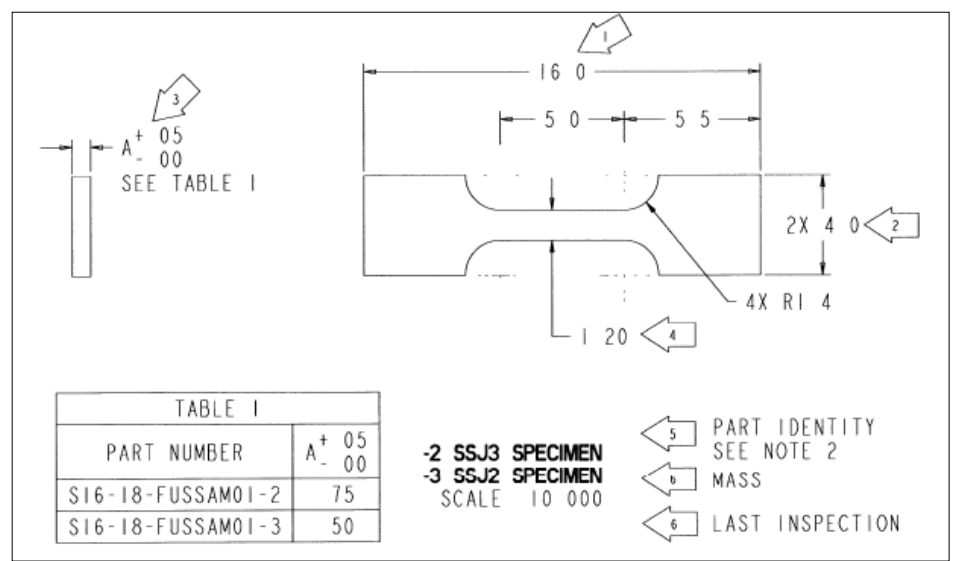

Fig. 1. SS-J3 tensile specimen schematic.

For bend bad fracture toughness, the test matrix, summarized in Table 3, includes ten variants of Eurofer-97 materials with one material, Group E, tested at two different heat treatment conditions.

Table 3. Eurofer-97 fracture toughness testing matrix.

\begin{tabular}{|c|c|c|}
\hline Materials & Heat treatment condition & Specimen orientation \\
\hline Group H & As received & L-T \\
\hline Group I & As received & L-T \\
\hline Group J & As received & L-T \\
\hline Group K & As received & L-T \\
\hline Group L & As received & L-T \\
\hline Group M & As received & L-T \\
\hline Group N & As received & L-T \\
\hline Group O & As received & L-T \\
\hline Group P & As received & L-S \\
\hline \multirow{3}{*}{ Group E } & As received & L-S \\
\cline { 2 - 3 } & $\begin{array}{l}\text { 1. Homogenization at } 1050^{\circ} \mathrm{C} / 30 \mathrm{~min} / \text { air cool } \\
\text { 2. Normalization at } 980^{\circ} \mathrm{C} / 30 \mathrm{~min} / \text { air cool } \\
\text { 3. Tempering at } 760^{\circ} \mathrm{C} / 90 \mathrm{~min} / \text { air cool }\end{array}$ \\
\hline
\end{tabular}

Fig. 2 shows the miniature multi-notch bend bar M4CVN specimens with a dimension of $45 \mathrm{~mm}$ (length) $\times 3.3 \mathrm{~mm}$ (width) $\times 1.65 \mathrm{~mm}$ (thickness) to characterize the transition fracture toughness of the steels based on the ASTM E1921 Master Curve method [1]. 

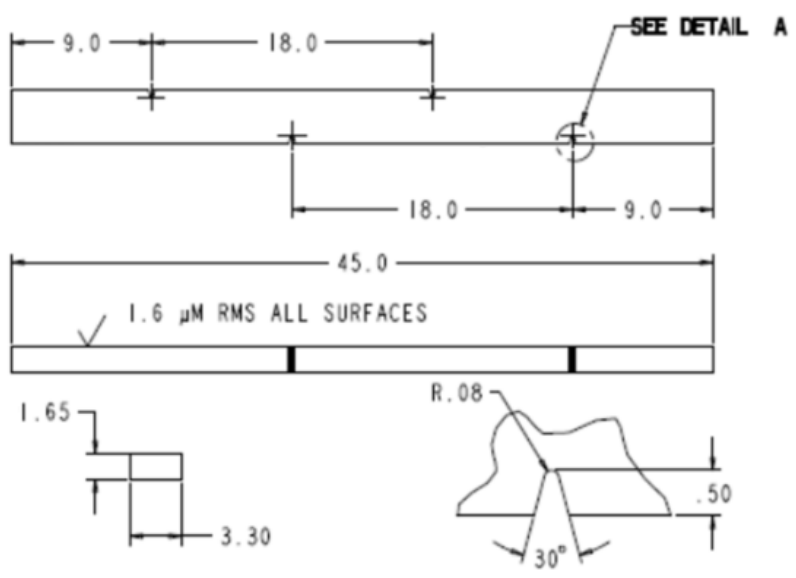

$\underset{\text { SCALE }}{\text { DET }}$ A A

Fig. 2 M4CVN specimen drawing. Units in millimeter.

The prerequisite of transition fracture toughness testing is having a sharp starting crack in the specimen which was realized by high frequency fatigue pre-cracking in this study. We performed fatigue pre-cracking on M4CVN specimens using a three-point bend type test fixture mounted on a $44.5 \mathrm{kN}$ capacity servo-hydraulic frame as shown in Fig. 3. The span to width ratio of the specimen was kept constant at four. During the fatigue pre-cracking process, the load-line compliance at the machined notch of the specimen was measured by a deflection gauge which provides the real-time crack length using the following equation from Ref. [2]:

$$
\begin{aligned}
& a / W=1.0005-4.1527 U+9.7477 U^{2}-214.2 U^{3}+1604.3 U^{4}-4633.4 U^{5} \\
& U=1 /\left\{\left[d E\left(B B_{n}\right)^{1 / 2} / P\right]^{1 / 2}+1\right\}
\end{aligned}
$$

where:

$a=$ crack length,

$d / P=$ measured load-line compliance,

$E=$ material Young's modulus,

$B_{n}=$ specimen net thickness (equals B for non-sidegrooved specimen),

$B=$ specimen thickness.

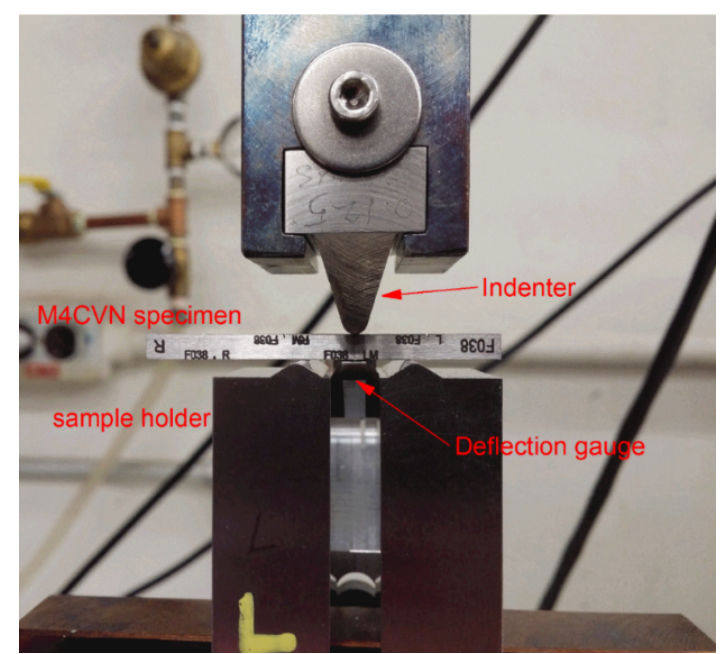

Fig. 3 Fatigue pre-cracking test fixture for a M4CVN specimen 
We applied the fatigue pre-cracking procedure in accordance with ASTM E1921 Standard. The standard sets limitations on both the maximum fatigue force $\left(\mathrm{P}_{\mathrm{m}}\right)$ as well as the allowable maximum stress intensity factor $\left(\mathrm{K}_{\max }\right)$ during the fatigue pre-cracking process. $\mathrm{P}_{\mathrm{m}}$ for the M4CVN specimen used in this study is defined per ASTM E1921:

$$
P_{m}=\frac{0.5 B b_{0}^{2} \sigma_{Y}}{S}
$$

where:

$B=$ specimen thickness,

$b_{0}=$ length of the initial uncracked ligament,

$\sigma_{Y}=$ average of material yield and tensile strengths,

$S=$ span distance.

After substituting the specimen dimensions and typical Eurofer-97 yield strength (560 MPa) and tensile strength $(670 \mathrm{MPa})$ into Eq. (2), $\mathrm{P}_{\mathrm{m}}$ for the M4CVN specimen is approximately $302.5 \mathrm{~N}$ (68 lbs). During fatigue pre-cracking, we applied a constant stress intensity in the range of 11.3-12.3 MPa $\sqrt{\mathrm{m}}$ which was well below the allowable $\mathrm{K}_{\max }$ per ASTM E1921. In addition, the maximum fatigue force was also less than $\mathrm{P}_{\mathrm{m}}$. The fatigue pre-cracking frequency was $45-50 \mathrm{~Hz}$ and the minimum to maximum fatigue force ratio, R-ratio, was kept constant at 0.1 . In general, it took approximately 200-400 thousand cycles for completing fatigue pre-cracking of one notch to a crack size to width $(\mathrm{a} / \mathrm{W})$ ratio of $0.44-0.5$.

The test frame used for fracture toughness testing was a $222.4 \mathrm{kN}$ capacity servo-hydraulic frame with a calibrated $4.45 \mathrm{kN}$ capacity load cell. Fig. 4 illustrates the general layout of the experimental setup. We used liquid nitrogen to control testing temperatures which were measured directly from type-T thermocouple wires spot welded to specimens. The environment chamber enclosed specimens and the test setup was used to maintain a relatively stable temperature during testing. As shown in Fig. 5, the M4CVN specimen test setup consists of a specimen indenter and a specimen fixture. The deflection gauge attached to the specimen fixture was used to measure the load-line displacement of the specimen. The push bar can slide left and right and is used to push the specimen against the positioning block such that the specimen notch is aligned with the specimen indenter and the deflection gauge. 


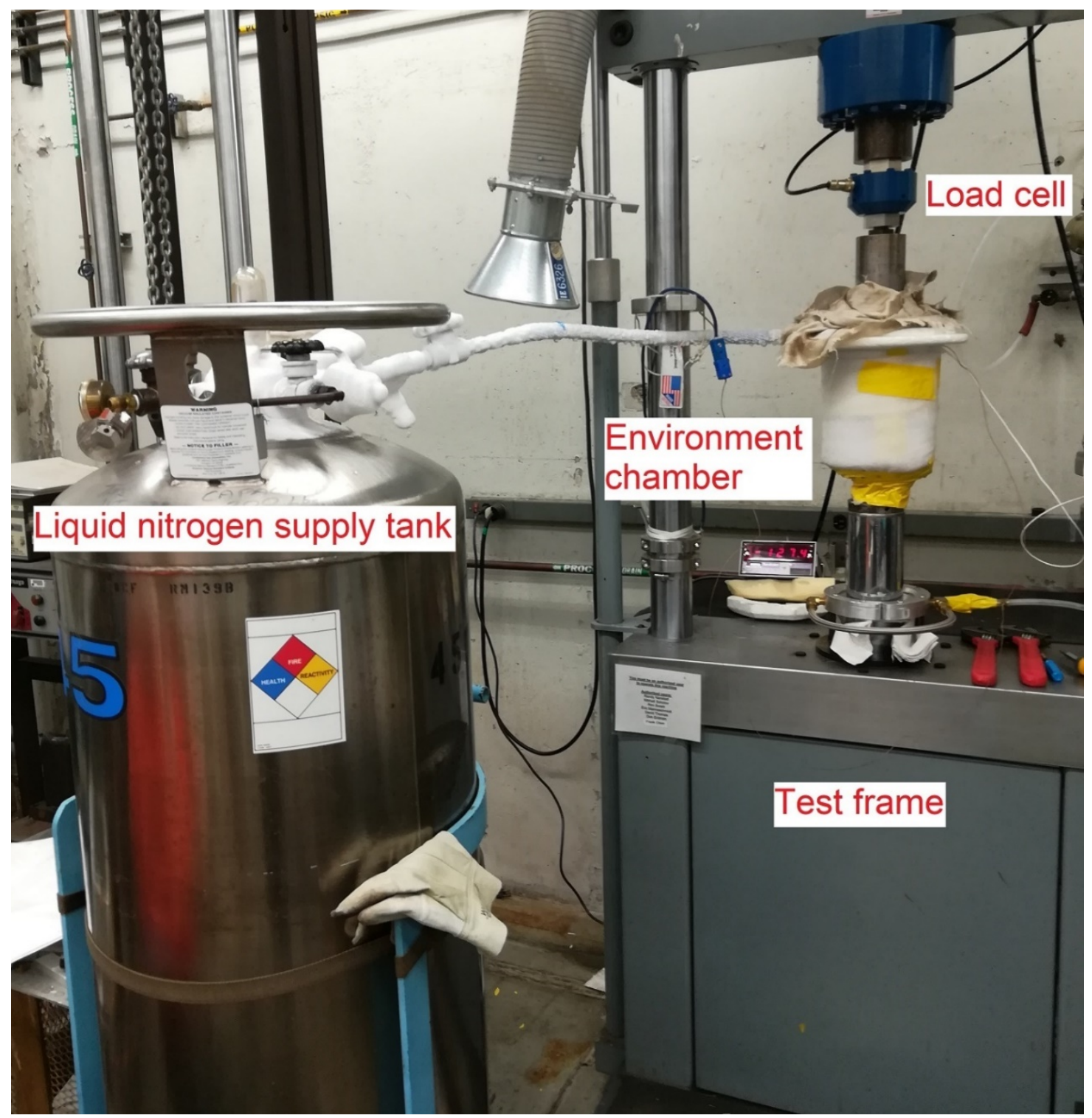

Fig. 4 General layout of the fracture toughness test setup 


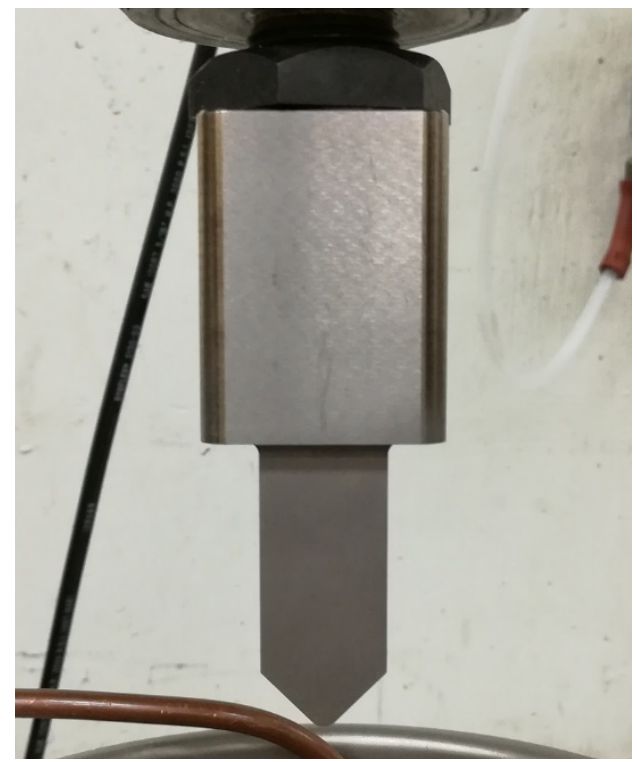

(a)

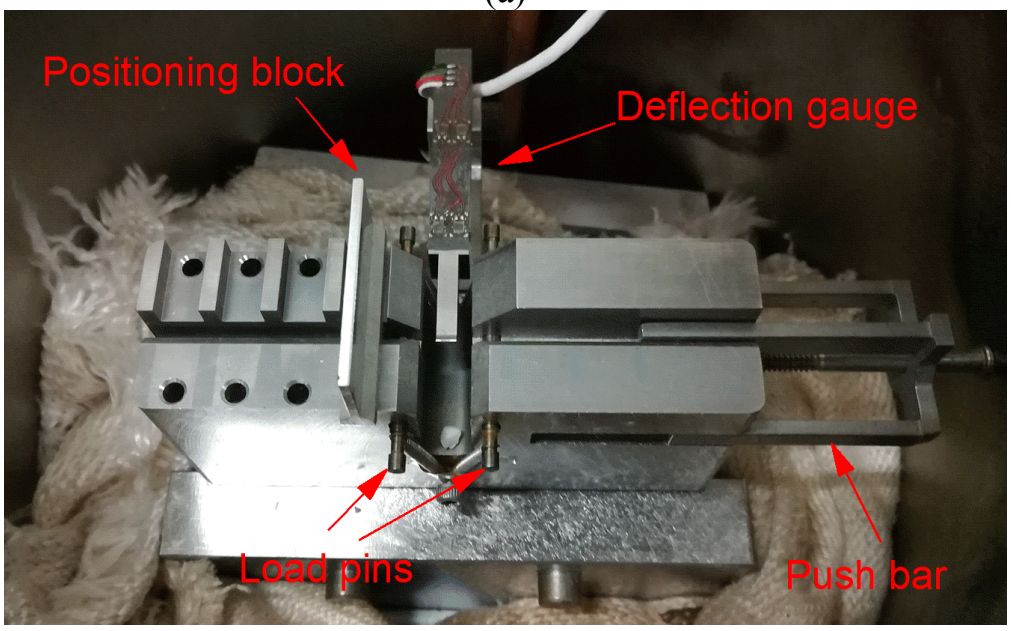

(b)

Fig. 5 M4CVN specimen test setup: specimen indenter in (a) and specimen fixture in (b)

We performed fracture toughness testing according to the ASTM E1921 Master Curve method. The test temperatures were selected by balancing between obtaining as high fracture toughness results as possible and still within the fracture toughness capacity limit $\mathrm{K}_{\text {Jclimit }}$ given in Eq. (3):

where:

$$
K_{J c \lim i t}=\sqrt{\frac{E b_{O} \sigma_{Y S}}{30\left(1-v^{2}\right)}}
$$

$E=$ material Young's modulus at the test temperature,

$b_{0}=$ length of the initial uncracked ligament,

$\sigma_{Y S}=$ material yield strength at the test temperature,

$v=$ Poisson's ratio.

Each specimen notch was tested until cleavage and then the crack length was measured from the fracture surface. The elastic-plastic equivalent stress intensity factor, $\mathrm{K}_{\mathrm{Jc}}$, was derived from the J-integral at the onset of cleavage fracture and size-adjusted to $1 \mathrm{~T}$ value based on the statistical weakest-link theory:

$$
K_{J C(1 T)}=20+\left[K_{J C(o)}-20\right]\left(\frac{B_{0}}{B_{1 T}}\right)^{1 / 4}
$$


where:

$K_{J c(1 T)}=\mathrm{K}_{\mathrm{Jc}}$ for a specimen thickness of one inch $\left(\mathrm{B}_{1 \mathrm{~T}}=25.4 \mathrm{~mm}\right)$,

$K_{J c(o)}=\mathrm{K}_{\mathrm{Jc}}$ for a specimen thickness of $\mathrm{B}_{\mathrm{o}}\left(\mathrm{B}_{\mathrm{o}}=1.641 \mathrm{~mm}\right.$ for M4CVN specimens $)$.

We then calculated the Master Curve provisional reference temperature $\mathrm{T}_{\mathrm{oQ}}$ using multitemperature analysis method in Eq. (5) and $\mathrm{K}_{\mathrm{Jc}}$ data were censored against both the fracture toughness capacity limit $\mathrm{K}_{\mathrm{Jclimit}}$ and the slow stable crack growth limit $\mathrm{K}_{\mathrm{Jc} \Delta \mathrm{a}}$.

$$
\begin{aligned}
& \sum_{i=1}^{N} \delta_{i} \frac{\exp \left[0.019\left(T_{i}-T_{o Q}\right)\right]}{11.0+76.7 \exp \left[0.019\left(T_{i}-T_{o Q}\right)\right]} \\
& -\sum_{i=1}^{N} \frac{\left(K_{J c(i)}-20\right)^{4} \exp \left[0.019\left(T_{i}-T_{o Q}\right)\right]}{\left\{11.0+76.7 \exp \left[0.019\left(T_{i}-T_{o Q}\right)\right]\right\}^{5}}=0
\end{aligned}
$$

where:

$N=$ number of specimens tested,

$T_{i}=$ test temperature corresponding to $\mathrm{K}_{\mathrm{Jc}(\mathrm{i})}$,

$K_{J c(i)}=$ either a valid $\mathrm{K}_{\mathrm{Jc}}$ datum or a datum replaced with a censoring value,

$\delta_{i}=1.0$ if the datum is valid or zero if the datum is a censored value,

$T_{o Q}=$ Master Curve provisional reference temperature solved by iteration.

\subsubsection{Microstructure characterization}

Microstructure of the as-received samples was characterized at multi length scales using light optical microscopy (LOM), scanning electron microscopy (SEM) and transmission/scanning-transmission electron microscopy (TEM/STEM). As a first step, LOM was performed to observe the grain/lath structures, inclusions and to verify any heterogeneities existing in the material that may affect the irradiation behavior or the evolution of the mechanical properties. One sample per steel was analysed by LOM. The characterization was performed on the undeformed head/grip sections of the broken tensile samples after the tensile tests. Samples were mounted in conductive bakelite and then grinded/polished to obtain mirror polish surface finish. Final polishing was performed using colloidal silica for EBSD quality finish. Following the polishing step, the samples were etched in Villela's reagent (picric acid + hydrochloric acid + ethanol) to reveal the microstructure features. All the steels were etched using the same etchant, which is known to work well with stainless steels. Then optical images were recorded from the etched sample surface along the rolling direction, representing the rolling direction - normal direction plane (RD-ND plane).

SEM imaging was performed on the same etched samples on which LOM was conducted, to record a broad overview of the steel microstructures at SEM length scales, especially if there were inclusions in the steel. Further, SEM imaging was also performed to observe the fracture surface of the samples after the room temperature tensile tests. For these experiments, a field emission gun (FEG) based Hitachi S4800 SEM was used. One sample per steel was analysed.

TEM/STEM characterization of the samples was also performed on focused ion beam (FIB) lift out specimens. The FIB lift outs were prepared from the undeformed head sections of the broken tensile samples. For this purpose, a tungsten filament based FEI Quanta DualBeam FIB-SEM was used. Initial lift out was performed using $30 \mathrm{kV}$ gallium $(\mathrm{Ga})$ ions. While thinning, the energy and current of the Ga beam was reduced progressively to minimize milling induced damage to the thin foils. Final polishing was performed using $2 \mathrm{kV}$ Ga ions for 10 minutes on each sample surface. The lift outs were extracted in a way that the electron transparent surface was along the RD-ND plane. Two samples per steel samples were imaged.

TEM/STEM imaging was performed on the FIB samples using a XFEG based FEI F200X Talos STEM operating at $200 \mathrm{kV}$, equipped with high resolution STEM detectors and a FEI 4096×4096 resolution 
"Ceta" CCD camera. Even after tempering, F-M steels contain high dislocation densities. Therefore, imaging of precipitate phases in F-M steels is tedious using conventional TEM/STEM because both dislocations and precipitates are imaged close to diffraction conditions (either two-beam or down-axis). Due to this, small precipitates can be masked by the overlapping contrast of the dislocations. Moreover, because of the similar atomic number $(\mathrm{Z})$ of the constituents of $\mathrm{M}_{23} \mathrm{C}_{6} / \mathrm{M}_{7} \mathrm{C}_{3}$ carbides and the steel matrix $(\mathrm{Fe} / \mathrm{Cr})$, these carbides do not show a very sharp contrast in TEM/STEM images. To overcome these two typical issues encountered in F-M steels, the precipitates were further imaged using high-count rate EDX mapping of the samples using FEI F200X Talos STEM. This method also avoided the error prone task of differentiating between FIB milling induced defects and small precipitates. The EDX maps were recorded for 1 hour for each sample, where the average count rate varied between $80-110 \mathrm{k}$ counts per sec.

\section{RESULTS}

\subsection{Mechanical properties}

\subsubsection{Room temperature Vickers hardness tests}

Vickers hardness indentation tests were performed on all the steel variants on the head/grip section of SS-J3 tensile samples. These tests were conducted in accordance with ASTM E384 standard using $1 \mathrm{~kg}$ load, $15 \mathrm{~s}$ dwell time. At least ten indents were measured for each steel (five per sample), and forty-seven indents on nine different samples for the reference E series. Fig. 6 shows the hardness variation of the different steel samples.

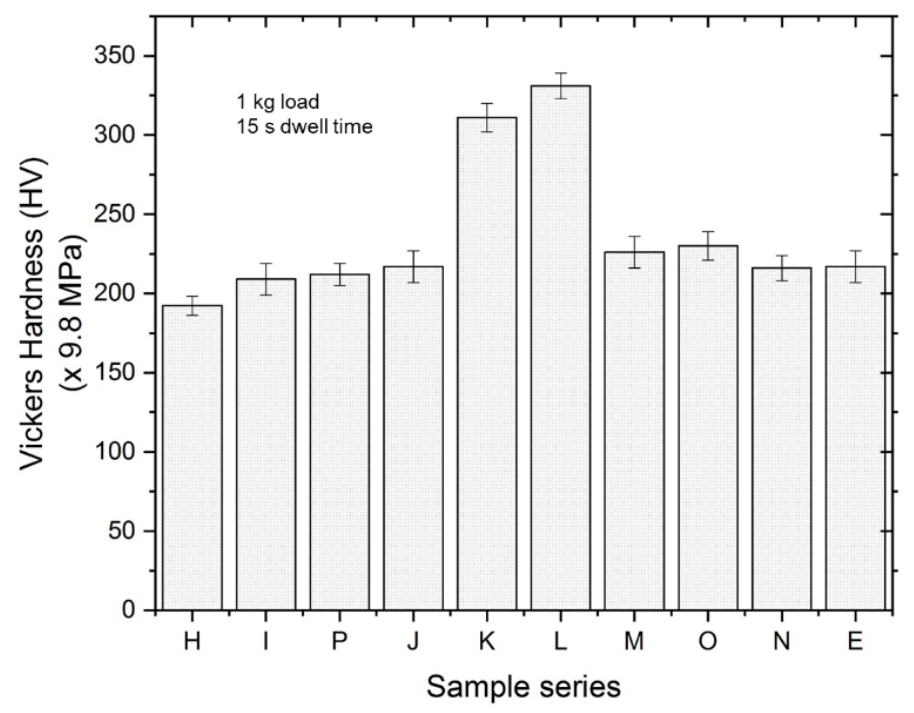

Fig. 6. Vickers hardness tests results performed on SS-J3 head/grip sections.

It is evident from the results that both $\mathrm{K}$ and $\mathrm{L}$ series steels had the highest hardness, exceeding $300 \mathrm{HV}$, while all the other steels did not deviate significantly when compared with the reference E series. We attribute this to the much lower tempering temperatures for both these steels, despite having slightly different chemistry and normalizing conditions. L series was tempered at $700{ }^{\circ} \mathrm{C}$, while the $\mathrm{K}$ series steel was tempered at a temperature as low as $675^{\circ} \mathrm{C}$. Both these temperatures are lower than $760{ }^{\circ} \mathrm{C}$ tempering temperature used for reference Eurofer-97 steels or conventional $9 \% \mathrm{Cr}$ steels in general. $\mathrm{H}$ series steel from KIT batch was the softest, with an average Vickers hardness of only 192 HV.

Table 4 summarizes the hardness values from every test that was performed on the various steels. 
Table 4. Vickers hardness indentation results from every indent performed on the various steels.

\begin{tabular}{|c|c|c|c|c|c|c|}
\hline Sample id & \multicolumn{5}{|c|}{ Vickers Hardness (HV), x9.8 MPa } \\
\hline $\begin{array}{c}\text { G100 (old } \\
\text { reference) }\end{array}$ & 163.1 & 160.9 & 160 & 170 & 162.8 & - \\
\hline $\begin{array}{c}\text { G101 (old } \\
\text { reference) }\end{array}$ & 162.5 & 164.5 & 164.1 & 155 & 158 & - \\
\hline H100 & 183.2 & 194.6 & 187.3 & 193.8 & 198.5 & - \\
\hline H101 & 198.7 & 195.8 & 192.1 & 181.9 & 196.8 & - \\
\hline I100 & 200.3 & 210.7 & 201.4 & 209.4 & 211.2 & - \\
\hline I101 & 212.5 & 206.5 & 213.2 & 203.7 & 215 & - \\
\hline I102 & 225.4 & 210 & 210 & 211.4 & 203 & - \\
\hline J100 & 222.4 & 210.3 & 221 & 221.4 & 223.1 & - \\
\hline J101 & 200.3 & 232.1 & 206 & 211 & 212 & - \\
\hline J104 & 216.2 & 212.5 & 225.4 & 221 & 201.5 & - \\
\hline J105 & 216 & 227.1 & 223.1 & 207.2 & 225 & \\
\hline K100 & 305.5 & 293.7 & 320.2 & 308.3 & 320 & 305.9 \\
\hline K101 & 318.1 & 303.6 & 324.4 & 311.1 & 303.2 & - \\
\hline L100 & 334.1 & 342.7 & 324 & 349.4 & 308 & 328 \\
\hline L101 & 318.1 & 331 & 345 & 327 & 324 & 323.6 \\
\hline L102 & 339.5 & 311.1 & 320 & 342 & 342.3 & 333.2 \\
\hline M108 & 226.4 & 235 & 212.3 & 216 & 224 & - \\
\hline M109 & 227.4 & 231.2 & 228 & 235 & 232.2 & - \\
\hline M110 & 206.2 & 213 & 213 & 222.2 & 235.9 & 226 \\
\hline M111 & 230.4 & 225.6 & 242 & 233.3 & 221.2 & 229 \\
\hline N108 & 206 & 202 & 217.4 & 193 & 216 & 227 \\
\hline N109 & 221 & 210 & 219 & 215.5 & 228 & 219 \\
\hline O108 & 219 & 235.4 & 232.5 & 220 & 240 & 234.1 \\
\hline O109 & 238 & 233 & 224 & 230.2 & 237.5 & - \\
\hline P100 & 207.8 & 217.4 & 206 & 214 & 219 & - \\
\hline P101 & 209.2 & 213 & 207.4 & 211.6 & 213.6 & - \\
\hline E300 & 206.8 & 212.2 & 215.7 & 235.4 & 213 & 220 \\
\hline E301 & 227 & 215.1 & 202.5 & 202.5 & 204.4 & 217.7 \\
\hline E302 & 224.2 & 229.2 & 214.4 & 209.6 & 216.7 & - \\
\hline E303 & 223 & 218.7 & 220.4 & 222.8 & 219.4 & - \\
\hline E304 & 211.5 & 225.3 & 224.2 & 223.2 & 221.4 & - \\
\hline E305 & 216.7 & 210.6 & 217.7 & 217.4 & 206 & - \\
\hline E306 & 212.5 & 217.4 & 219.4 & 225 & 219.7 & - \\
\hline E307 & 217.4 & 209 & 227.4 & 215.1 & 219.7 & - \\
\hline E308 & 213.1 & 226.3 & 228.5 & 219.1 & 216.7 & - \\
\hline
\end{tabular}

\subsubsection{Uniaxial tensile tests}

Using a strain rate of $10^{-3} \mathrm{~s}^{-1}$, room temperature tensile tests were performed on the SS-J3 tensile samples, in shoulder loading configuration and using the machine stroke for estimating elongation. The 
summary of each test result is tabulated in Table 5. Because of machine compliance, we have separately reported the non-linear plastic strain information that remains is unaffected by the compliance. Reduction in area (RA) after the tensile tests was calculated using SEM imaging on the fracture surface. For convenience, RA values are also reported in Table 5, while fractography of the steels is presented in the next sections. The engineering stress-strain curves for all the steel variants is shown in Fig. 7.

Table 5. Summary of room temperature tensile tests.

\begin{tabular}{|c|c|c|c|c|c|c|c|c|}
\hline \multirow{2}{*}{$\begin{array}{l}\text { Specimen } \\
\text { Id. }\end{array}$} & \multicolumn{2}{|c|}{ Elongation (\%) } & \multicolumn{2}{|c|}{ Plastic elongation $(\%)$} & \multicolumn{2}{|c|}{ Strength (MPa) } & \multirow{3}{*}{$\begin{array}{c}\text { RA (\%) } \\
83.6\end{array}$} & \multirow{2}{*}{$\begin{array}{c}\text { Test } \\
\text { Temperature } \\
\left({ }^{\circ} \mathrm{C}\right)\end{array}$} \\
\hline & Uniform & Total & Uniform & Total & YS & UTS & & \\
\hline H104 & 17.30 & 37.50 & 13.27 & 36.55 & 403.34 & 548.13 & & 23 \\
\hline H105 & 15.80 & 35.78 & 11.97 & 34.15 & 399.90 & 548.13 & & 23 \\
\hline I110 & 13.6 & 33.64 & 9.50 & 31.60 & 472.29 & 603.29 & \multirow{2}{*}{81.3} & 23 \\
\hline I111 & 15.90 & 34.00 & 12.04 & 32.32 & 461.95 & 603.29 & & 23 \\
\hline J101 & 12.43 & 29.50 & 9.30 & 28.14 & 492.98 & 630.87 & \multirow{2}{*}{82.6} & 23 \\
\hline J110 & 11.76 & 30.33 & 8.60 & 29.78 & 489.53 & 625.70 & & 23 \\
\hline K103 & 6.93 & 18.79 & 3.13 & 17.11 & 804.96 & 868.74 & \multirow{2}{*}{82.7} & 23 \\
\hline K107 & 6.39 & 20.00 & 2.57 & 18.43 & 799.79 & 854.95 & & 23 \\
\hline L104 & 7.70 & 20.30 & 3.30 & 17.80 & 871.50 & 958.37 & \multirow{2}{*}{74.7} & 23 \\
\hline L105 & 9.20 & 20.30 & 4.90 & 17.90 & 863.57 & 961.82 & & 23 \\
\hline M113 & 8.92 & 28.03 & 6.12 & 26.65 & 544.69 & 668.79 & \multirow{2}{*}{79.5} & 23 \\
\hline M118 & 11.25 & 25.80 & 8.74 & 24.50 & 558.48 & 689.48 & & 23 \\
\hline N113 & 9.12 & 25.90 & 5.26 & 23.04 & 522.62 & 627.42 & \multirow{2}{*}{84.3} & 23 \\
\hline N114 & 12.20 & 30.20 & 6.40 & 27.60 & 503.32 & 606.74 & & 23 \\
\hline 0113 & 9.79 & 24.40 & 6.10 & 22.60 & 581.92 & 684.30 & \multirow{2}{*}{79.2} & 23 \\
\hline 0119 & 8.40 & 23.40 & 4.95 & 21.92 & 570.54 & 679.13 & & 23 \\
\hline P104 & 15.46 & 32.70 & 11.57 & 30.90 & 478.50 & 618.80 & \multirow{2}{*}{81.1} & 23 \\
\hline P105 & 11.67 & 33.09 & 7.87 & 31.76 & 486.08 & 616.39 & & 23 \\
\hline E244 & 9.7 & 20.9 & 6.08 & 17.74 & 486.3 & 585.7 & \multirow{2}{*}{82.3} & 23 \\
\hline E246 & 10.4 & 22.9 & 6.9 & 19.4 & 461.1 & 569 & & 23 \\
\hline
\end{tabular}

As expected previously from the Vickers hardness results (Fig. 6), K and L series steels were the strongest, having the highest yield strength (YS) and ultimate tensile strength (UTS) above $800 \mathrm{MPa}$, but appeared to have low toughness (area under the stress-strain curves). Rest all other steels were not drastically different from each other in terms of tensile results. Among the KIT batch of H, I and P series steels, $\mathrm{H}$ was the softest having yield strength of $\sim 400 \mathrm{MPa}$ but with higher ductility. For I and P, yield strength was similar ranging between $460-485 \mathrm{MPa}$ range. J series steel, originating from SCK.CEN, had similar tensile properties to the I and P series of KIT batch despite having different heat treatments and slightly different chemistry. Among the double austenized versions of Eurofer-97 steels from ENEA, M had a higher yield strength ranging between $545-558 \mathrm{MPa}$, while the same for $\mathrm{N}$ was $500-523 \mathrm{MPa}$. This difference is perhaps primarily due to a lower concentration of carbon in $\mathrm{N}$ series compared to $\mathrm{M}$, despite 
having different austenization temperatures. $\mathrm{O}$ series steel, which was hot rolled at $650{ }^{\circ} \mathrm{C}$ to $40 \%$ reduction had higher yield strength (571-582 $\mathrm{MPa}$ ) and slightly lower elongation. It is important to highlight that despite the new heat treatment given to the out of spec Eurofer-97 reference bar (old G/F series), tensile tests revealed that the steel was still softer than results known for Eurofer-97.
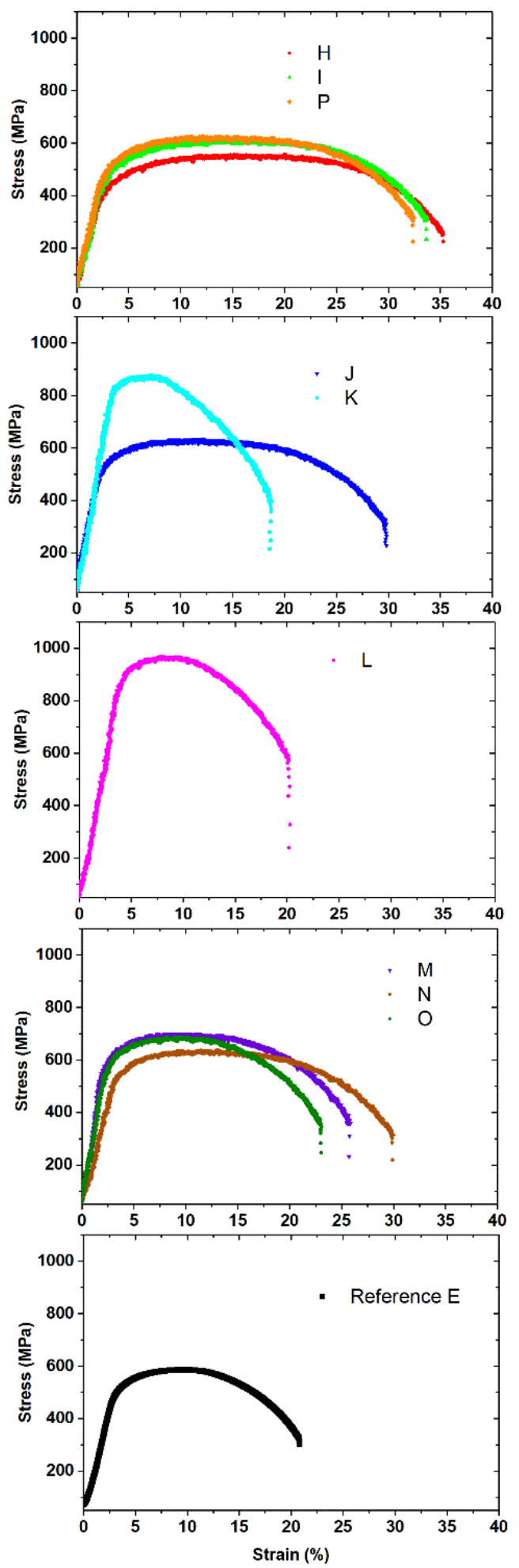

Fig. 7. Engineering stress-strain curves for the Eurofer-97 steel variants. For convenience only one curve per samples is shown. 


\subsubsection{Bend bar fracture toughness}

The fracture toughness results of ten variants of Eurofer-97steels are summarized in Table 7-17. Figs. 8-18 show the test data along with the derived Maser Curve. The equation for the Master Curve is:

$$
K_{J C(\text { med })}=30+70 \exp \left[0.019\left(T-T_{o Q}\right)\right]
$$

where:

$K_{J c(\text { med })}=$ median fracture toughness for a multi-temperature data set from 1T size specimen, $T=$ test temperature,

$T_{o Q}=$ Master Curve provisional reference temperature.

The tolerance bounds were calculated using the equation below:

where:

$$
K_{J c(0 . x x)}=20+\left[\ln \left(\frac{1}{1-0 . x x}\right)\right]^{1 / 4}\left\{11+77 \exp \left[0.019\left(T-T_{o Q}\right)\right]\right\}
$$

$0 . x x=$ selected cumulative probability level, e.g., for the $2 \%$ tolerance bound, $0 . x x=0.02$.

\begin{tabular}{|c|c|c|c|c|c|c|}
\hline $\begin{array}{l}\text { Specimen } \\
\text { ID }\end{array}$ & $\begin{array}{c}\text { Test } \\
\text { temperature } \\
\left({ }^{\circ} \mathrm{C}\right)\end{array}$ & $\begin{array}{c}\mathrm{K}_{\mathrm{Jc}} \\
(\mathrm{MPa} \sqrt{\mathrm{m}})\end{array}$ & $\begin{array}{c}1 \mathrm{~T}-\mathrm{K}_{\mathrm{Jc}} \\
(\mathrm{MPa} \sqrt{\mathrm{m}})\end{array}$ & $\begin{array}{c}\text { Censored } \\
(\mathrm{Y} / \mathrm{N})\end{array}$ & $\begin{array}{l}\text { Censored } \\
1 \mathrm{~T} \text { value } \\
(\mathrm{MPa} \sqrt{\mathrm{m}})\end{array}$ & $\mathrm{T}_{\mathrm{oQ}}\left({ }^{\circ} \mathrm{C}\right)$ \\
\hline H006L & -165 & 40.5 & 30.3 & $\mathrm{~N}$ & NA & \multirow{15}{*}{-89} \\
\hline H006LM & -154 & 204.8 & 113.2 & $\mathrm{Y}$ & 56.6 & \\
\hline H006RM & -165 & 63.1 & 41.7 & $\mathrm{~N}$ & NA & \\
\hline H006R & -165 & 71.8 & 46.1 & $\mathrm{~N}$ & NA & \\
\hline H002L & -159 & 68.0 & 44.2 & $\mathrm{~N}$ & NA & \\
\hline H002RM & -154 & 179.7 & 100.5 & $\mathrm{Y}$ & 56.6 & \\
\hline H002R & -158 & 51.1 & 35.7 & $\mathrm{~N}$ & NA & \\
\hline H003L & -154 & 89.9 & 55.2 & $\mathrm{~N}$ & $\mathrm{NA}$ & \\
\hline H003LM & -154 & 33.0 & 26.6 & $\mathrm{~N}$ & $\mathrm{NA}$ & \\
\hline H003RM & -154 & 64.2 & 42.3 & $\mathrm{~N}$ & $\mathrm{NA}$ & \\
\hline H003R & -152 & 89.4 & 55.0 & $\mathrm{~N}$ & $\mathrm{NA}$ & \\
\hline H004L & -154 & 92.6 & 56.6 & $\mathrm{~N}$ & NA & \\
\hline H004LM & -155 & 53.2 & 36.7 & $\mathrm{~N}$ & NA & \\
\hline H004RM & -155 & 237.5 & 129.7 & $\mathrm{Y}$ & 56.6 & \\
\hline H004R & -148 & 301.3 & 161.8 & $\mathrm{Y}$ & 56.6 & \\
\hline
\end{tabular}

Table 7. Fracture toughness of Eurofer-97 Group $\mathrm{H}$ in the as-received condition

Table 8. Fracture toughness of Eurofer-97 Group I in the as-received condition

\begin{tabular}{|c|c|c|c|c|c|c|}
\hline $\begin{array}{c}\text { Specimen } \\
\text { ID }\end{array}$ & $\begin{array}{c}\text { Test } \\
\text { temperature } \\
\left({ }^{\circ} \mathrm{C}\right)\end{array}$ & $\begin{array}{c}\mathrm{K}_{\mathrm{Jc}} \\
(\mathrm{MPa} \sqrt{\mathrm{m}})\end{array}$ & $\begin{array}{c}1 \mathrm{~T}-\mathrm{K}_{\mathrm{Jc}} \\
(\mathrm{MPa} \sqrt{\mathrm{m}})\end{array}$ & $\begin{array}{c}\text { Censored } \\
(\mathrm{Y} / \mathrm{N})\end{array}$ & $\begin{array}{c}\text { Censored } \\
1 \mathrm{~T} \text { value } \\
(\mathrm{MPa} \sqrt{\mathrm{m}})\end{array}$ & \multirow{2}{*}{$\mathrm{T}_{\mathrm{oQ}}\left({ }^{\circ} \mathrm{C}\right)$} \\
\hline I006L & -165 & 54.7 & 37.5 & $\mathrm{~N}$ & $\mathrm{NA}$ & \\
\hline I006LM & -165 & 33.9 & 27.0 & $\mathrm{~N}$ & $\mathrm{NA}$ \\
\hline I006RM & -165 & 39.5 & 29.8 & $\mathrm{~N}$ & $\mathrm{NA}$ \\
\hline I006R & -157 & 52.4 & 36.3 & $\mathrm{~N}$ & $\mathrm{NA}$ & \multirow{2}{*}{-78} \\
\hline I005L & -124 & 280.5 & 151.3 & $\mathrm{Y}$ & 56.5 & \\
\hline I005LM & -126 & 292.5 & 157.4 & $\mathrm{Y}$ & 56.8 & \\
\hline I005RM & -126 & 76.0 & 48.2 & $\mathrm{~N}$ & $\mathrm{NA}$ \\
\hline I002L & -146 & 144.8 & 82.9 & $\mathrm{Y}$ & 58.8 \\
\hline I002LM & -146 & 114.5 & 67.6 & $\mathrm{Y}$ & 58.1 & \\
\hline I002RM & -146 & 36.4 & 28.3 & $\mathrm{~N}$ & $\mathrm{NA}$ & \\
\hline
\end{tabular}




\begin{tabular}{|c|c|c|c|c|c|}
\hline I002R & -146 & 93.8 & 57.2 & $\mathrm{~N}$ & NA \\
\hline $\mathrm{I} 003 \mathrm{~L}$ & -148 & 15.0 & 17.5 & $\mathrm{~N}$ & NA \\
\hline I003LM & -151 & 55.1 & 37.7 & $\mathrm{~N}$ & NA \\
\hline I003RM & -152 & 50.3 & 35.3 & $\mathrm{~N}$ & NA \\
\hline I003R & -146 & 254.9 & 138.4 & $\mathrm{Y}$ & 57.2 \\
\hline
\end{tabular}

Table 9. Fracture toughness of Eurofer-97 Group $\mathrm{J}$ in the as-received condition

\begin{tabular}{|c|c|c|c|c|c|c|}
\hline $\begin{array}{l}\text { Specimen } \\
\text { ID }\end{array}$ & $\begin{array}{c}\text { Test } \\
\text { temperature } \\
\left({ }^{\circ} \mathrm{C}\right)\end{array}$ & $\begin{array}{c}\mathrm{K}_{\mathrm{Jc}} \\
(\mathrm{MPa} \sqrt{\mathrm{m}})\end{array}$ & $\begin{array}{c}1 \mathrm{~T}-\mathrm{K}_{\mathrm{Ic}} \\
(\mathrm{MPa} \sqrt{\mathrm{m}})\end{array}$ & $\begin{array}{c}\text { Censored } \\
(\mathrm{Y} / \mathrm{N})\end{array}$ & $\begin{array}{l}\text { Censored } \\
1 \mathrm{~T} \text { value } \\
(\mathrm{MPa} \sqrt{\mathrm{m}})\end{array}$ & $\mathrm{T}_{\mathrm{oQ}}\left({ }^{\circ} \mathrm{C}\right)$ \\
\hline J001L & -168 & 59.0 & 39.7 & $\mathrm{~N}$ & NA & \multirow{19}{*}{-99} \\
\hline J001LM & -165 & 55.8 & 38.0 & $\mathrm{~N}$ & NA & \\
\hline J001RM & -165 & 59.0 & 39.7 & $\mathrm{~N}$ & NA & \\
\hline $\mathrm{J} 001 \mathrm{R}$ & -165 & 65.2 & 42.8 & $\mathrm{~N}$ & NA & \\
\hline $\mathrm{J} 002 \mathrm{~L}$ & -161 & 56.6 & 38.5 & $\mathrm{~N}$ & NA & \\
\hline J002LM & -159 & 86.4 & 53.5 & $\mathrm{~N}$ & NA & \\
\hline $\mathrm{J} 002 \mathrm{RM}$ & -159 & 45.7 & 33.0 & $\mathrm{~N}$ & NA & \\
\hline $\mathrm{J} 002 \mathrm{R}$ & -156 & 244.2 & 133.0 & $\mathrm{Y}$ & 53.5 & \\
\hline $\mathrm{J} 005 \mathrm{~L}$ & -149 & 201.3 & 111.4 & $\mathrm{Y}$ & 53.5 & \\
\hline J005RM & -136 & 231.2 & 126.5 & $\mathrm{Y}$ & 53.5 & \\
\hline J005R & -130 & 233.2 & 127.5 & $\mathrm{Y}$ & 53.5 & \\
\hline $\mathrm{J} 003 \mathrm{~L}$ & -155 & 166.1 & 93.7 & $\mathrm{Y}$ & 53.5 & \\
\hline J003LM & -156 & 162.6 & 91.9 & $\mathrm{Y}$ & 53.5 & \\
\hline $\mathrm{J} 003 \mathrm{RM}$ & -151 & 254.4 & 138.2 & $Y$ & 53.5 & \\
\hline J003R & -154 & 81.0 & 50.8 & $\mathrm{~N}$ & NA & \\
\hline J006L & -154 & 120.0 & 70.4 & $\mathrm{Y}$ & 60.1 & \\
\hline J006LM & -153 & 102.4 & 61.5 & $\mathrm{Y}$ & 60.5 & \\
\hline J006RM & -153 & 71.3 & 45.9 & $\mathrm{~N}$ & NA & \\
\hline J006R & -154 & 179.3 & 100.3 & $\mathrm{Y}$ & 53.5 & \\
\hline
\end{tabular}

Table 10. Fracture toughness of Eurofer-97 Group K in the as-received condition

\begin{tabular}{|c|c|c|c|c|c|c|}
\hline $\begin{array}{l}\text { Specimen } \\
\text { ID }\end{array}$ & $\begin{array}{c}\text { Test } \\
\text { temperature } \\
\left({ }^{\circ} \mathrm{C}\right)\end{array}$ & $\begin{array}{c}\mathrm{K}_{\mathrm{Jc}} \\
(\mathrm{MPa} \sqrt{\mathrm{m}})\end{array}$ & $\begin{array}{c}1 \mathrm{~T}-\mathrm{K}_{\mathrm{Jc}} \\
(\mathrm{MPa} \sqrt{\mathrm{m}})\end{array}$ & $\begin{array}{c}\text { Censored } \\
(\mathrm{Y} / \mathrm{N})\end{array}$ & $\begin{array}{l}\text { Censored } \\
1 \mathrm{~T} \text { value } \\
(\mathrm{MPa} \sqrt{\mathrm{m}})\end{array}$ & $\mathrm{T}_{\mathrm{oQ}}\left({ }^{\circ} \mathrm{C}\right)$ \\
\hline K007L* & -147 & 23.2 & 21.6 & $\mathrm{~N}$ & NA & \multirow{14}{*}{-12} \\
\hline K007LM* & -161 & 14.1 & 17.0 & $\mathrm{~N}$ & NA & \\
\hline K007RM* & -165 & 23.1 & 21.6 & $\mathrm{~N}$ & NA & \\
\hline K002L & -97 & 31.2 & 25.6 & $\mathrm{~N}$ & NA & \\
\hline K002LM & -96 & 83.8 & 52.2 & $\mathrm{~N}$ & NA & \\
\hline K002RM & -109 & 34.3 & 27.2 & $\mathrm{~N}$ & NA & \\
\hline K005L & -84 & 42.3 & 31.2 & $\mathrm{~N}$ & NA & \\
\hline K005LM & -84 & 106.5 & 63.6 & $\mathrm{Y}$ & 63.4 & \\
\hline K005RM & -84 & 44.4 & 32.3 & $\mathrm{~N}$ & NA & \\
\hline K005R & -89 & 289.4 & 155.8 & $\mathrm{Y}$ & 52.2 & \\
\hline K006L & -90 & 61.5 & 40.9 & $\mathrm{~N}$ & NA & \\
\hline K006LM & -90 & 56.3 & 38.3 & $\mathrm{~N}$ & NA & \\
\hline K006RM & -90 & 56.0 & 38.1 & $\mathrm{~N}$ & NA & \\
\hline K006R & -90 & 64.5 & 42.4 & $\mathrm{~N}$ & NA & \\
\hline
\end{tabular}

*Fracture toughness results for information only, not used for Master Curve calculation 
Table 11. Fracture toughness of Eurofer-97 Group L in the as-received condition

\begin{tabular}{|c|c|c|c|c|c|c|}
\hline $\begin{array}{l}\text { Specimen } \\
\text { ID }\end{array}$ & $\begin{array}{c}\text { Test } \\
\text { temperature } \\
\left({ }^{\circ} \mathrm{C}\right)\end{array}$ & $\begin{array}{c}\mathrm{K}_{\mathrm{Jc}} \\
(\mathrm{MPa} \sqrt{\mathrm{m}})\end{array}$ & $\begin{array}{c}1 \mathrm{~T}-\mathrm{K}_{\mathrm{Jc}} \\
(\mathrm{MPa} \sqrt{\mathrm{m}})\end{array}$ & $\begin{array}{c}\text { Censored } \\
(\mathrm{Y} / \mathrm{N})\end{array}$ & $\begin{array}{l}\text { Censored } \\
1 \mathrm{~T} \text { value } \\
(\mathrm{MPa} \sqrt{\mathrm{m}})\end{array}$ & $\mathrm{T}_{\mathrm{oQ}}\left({ }^{\circ} \mathrm{C}\right)$ \\
\hline L006L* & -137 & 21.8 & 20.9 & $\mathrm{~N}$ & NA & \multirow{16}{*}{-53} \\
\hline L006LM* & -149 & 21.9 & 20.9 & $\mathrm{~N}$ & $\mathrm{NA}$ & \\
\hline L006RM* & -153 & 25.2 & 22.6 & $\mathrm{~N}$ & $\mathrm{NA}$ & \\
\hline L006R* & -171 & 16.8 & 18.4 & $\mathrm{~N}$ & NA & \\
\hline L007L & -96 & 114.0 & 67.4 & $\mathrm{Y}$ & 67.3 & \\
\hline L007LM & -94 & 93.3 & 57.0 & $\mathrm{~N}$ & NA & \\
\hline L007RM & -94 & 73.3 & 46.9 & $\mathrm{~N}$ & $\mathrm{NA}$ & \\
\hline L007R & -95 & 89.7 & 55.1 & $\mathrm{~N}$ & NA & \\
\hline L002L & -90 & 149.7 & 85.4 & $\mathrm{Y}$ & 65.7 & \\
\hline L002LM & -90 & 110.6 & 65.7 & $\mathrm{~N}$ & NA & \\
\hline L002RM & -91 & 109.6 & 65.2 & $\mathrm{~N}$ & NA & \\
\hline L002R & -90 & 144.8 & 82.9 & $\mathrm{Y}$ & 67.2 & \\
\hline L004L & -89 & 145.7 & 83.4 & $\mathrm{Y}$ & 64.6 & \\
\hline L004LM & -92 & 50.1 & 35.1 & $\mathrm{~N}$ & NA & \\
\hline L004RM & -91 & 68.9 & 44.6 & $\mathrm{~N}$ & NA & \\
\hline L004R & -91 & 137.3 & 79.1 & $\mathrm{Y}$ & 67.3 & \\
\hline
\end{tabular}

*Fracture toughness results for information only, not used for Master Curve calculation

Table 12. Fracture toughness of Eurofer-97 Group M in the as-received condition

\begin{tabular}{|c|c|c|c|c|c|c|}
\hline $\begin{array}{l}\text { Specimen } \\
\text { ID }\end{array}$ & $\begin{array}{c}\text { Test } \\
\text { temperature } \\
\left({ }^{\circ} \mathrm{C}\right)\end{array}$ & $\begin{array}{c}\mathrm{K}_{\mathrm{Jc}} \\
(\mathrm{MPa} \sqrt{\mathrm{m}})\end{array}$ & $\begin{array}{c}1 \mathrm{~T}-\mathrm{K}_{\mathrm{Jc}} \\
(\mathrm{MPa} \sqrt{\mathrm{m}})\end{array}$ & $\begin{array}{c}\text { Censored } \\
(\mathrm{Y} / \mathrm{N})\end{array}$ & $\begin{array}{l}\text { Censored } \\
1 \mathrm{~T} \text { value } \\
(\mathrm{MPa} \sqrt{\mathrm{m}})\end{array}$ & $\mathrm{T}_{\mathrm{oQ}}\left({ }^{\circ} \mathrm{C}\right)$ \\
\hline M003L & -165 & 25.2 & 22.6 & $\mathrm{~N}$ & NA & \multirow{16}{*}{-75} \\
\hline M003LM & -165 & 83.8 & 52.2 & $\mathrm{~N}$ & $\mathrm{NA}$ & \\
\hline M003RM & -165 & 52.9 & 36.6 & $\mathrm{~N}$ & NA & \\
\hline M003R & -165 & 39.7 & 29.9 & $\mathrm{~N}$ & NA & \\
\hline M002L & -150 & 220.8 & 121.2 & $Y$ & 53.7 & \\
\hline M002LM & -150 & 58.7 & 39.5 & $\mathrm{~N}$ & $\mathrm{NA}$ & \\
\hline M002R & -142 & 194.6 & 108.0 & $\mathrm{Y}$ & 53.7 & \\
\hline M004L & -154 & 80.5 & 50.5 & $\mathrm{~N}$ & NA & \\
\hline M004LM & -154 & 40.3 & 30.2 & $\mathrm{~N}$ & NA & \\
\hline M004RM & -154 & 86.9 & 53.7 & $\mathrm{~N}$ & NA & \\
\hline M004R & -154 & 37.0 & 28.6 & $\mathrm{~N}$ & NA & \\
\hline M007L & -142 & 232.1 & 126.9 & $Y$ & 53.7 & \\
\hline M007LM & -142 & 78.0 & 49.2 & $\mathrm{~N}$ & NA & \\
\hline M007RM & -148 & 35.8 & 28.0 & $\mathrm{~N}$ & NA & \\
\hline M007R & -148 & 67.9 & 44.1 & $\mathrm{~N}$ & NA & \\
\hline $\mathrm{M} 003 \mathrm{~L}$ & -165 & 25.2 & 22.6 & $\mathrm{~N}$ & NA & \\
\hline
\end{tabular}

Table 13. Fracture toughness of Eurofer-97 Group N in the as-received condition

\begin{tabular}{|c|c|c|c|c|c|c|}
\hline $\begin{array}{c}\text { Specimen } \\
\text { ID }\end{array}$ & $\begin{array}{c}\text { Test } \\
\text { temperature } \\
\left({ }^{\circ} \mathrm{C}\right)\end{array}$ & $\begin{array}{c}\mathrm{K}_{\mathrm{Jc}} \\
(\mathrm{MPa} \sqrt{\mathrm{m}})\end{array}$ & $\begin{array}{c}1 \mathrm{~T}-\mathrm{K}_{\mathrm{Jc}} \\
(\mathrm{MPa} \sqrt{\mathrm{m}})\end{array}$ & $\begin{array}{c}\text { Censored } \\
(\mathrm{Y} / \mathrm{N})\end{array}$ & $\begin{array}{c}\text { Censored } \\
1 \mathrm{~T} \text { value } \\
(\mathrm{MPa} \sqrt{\mathrm{m}})\end{array}$ & \multirow{2}{*}{$\mathrm{T}_{\mathrm{oQ}}\left({ }^{\circ} \mathrm{C}\right)$} \\
\cline { 1 - 5 } N003L & -170 & 45.4 & 32.8 & $\mathrm{~N}$ & $\mathrm{NA}$ & \multirow{2}{*}{-92} \\
\hline N003LM & -170 & 32.9 & 26.5 & $\mathrm{~N}$ & $\mathrm{NA}$ & \\
\hline N003RM & -165 & 108.3 & 64.5 & $\mathrm{Y}$ & $\mathrm{N}$ & \\
\hline N003R & -171 & 35.0 & 27.6 & $\mathrm{~N}$ & $\mathrm{NA}$ & \\
\hline
\end{tabular}




\begin{tabular}{|c|c|c|c|c|c|} 
N006L & -152 & 43.7 & 31.9 & $\mathrm{~N}$ & NA \\
\hline N006LM & -151 & 67.7 & 44.0 & $\mathrm{~N}$ & NA \\
\hline N006RM & -154 & 117.0 & 68.9 & $\mathrm{Y}$ & 60.5 \\
\hline N006R & -151 & 80.4 & 50.5 & $\mathrm{~N}$ & NA \\
\hline N002L & -149 & 168.7 & 95.0 & $\mathrm{Y}$ & 58.6 \\
\hline N002LM & -150 & 96.6 & 58.6 & $\mathrm{~N}$ & NA \\
\hline N002RM & -151 & 64.7 & 42.5 & $\mathrm{~N}$ & NA \\
\hline N002R & -151 & 54.2 & 37.2 & $\mathrm{~N}$ & NA \\
\hline N005L & -152 & 87.7 & 54.1 & $\mathrm{~N}$ & NA \\
\hline N005LM & -153 & 85.8 & 53.2 & $\mathrm{~N}$ & NA \\
\hline N005RM & -153 & 226.0 & 123.9 & $\mathrm{Y}$ & 58.6 \\
\hline N005R & -151 & 65.9 & 43.1 & $\mathrm{~N}$ & NA \\
\hline
\end{tabular}

Table 14. Fracture toughness of Eurofer-97 Group O in the as-received condition

\begin{tabular}{|c|c|c|c|c|c|c|}
\hline $\begin{array}{c}\text { Specimen } \\
\text { ID }\end{array}$ & $\begin{array}{c}\text { Test } \\
\text { temperature } \\
\left({ }^{\circ} \mathrm{C}\right)\end{array}$ & $\begin{array}{c}\mathrm{K}_{\mathrm{Jc}} \\
(\mathrm{MPa} \sqrt{\mathrm{m}})\end{array}$ & $\begin{array}{c}1 \mathrm{~T}-\mathrm{K}_{\mathrm{Jc}} \\
(\mathrm{MPa} \sqrt{\mathrm{m}})\end{array}$ & $\begin{array}{c}\text { Censored } \\
(\mathrm{Y} / \mathrm{N})\end{array}$ & $\begin{array}{c}\text { Censored } \\
1 \mathrm{~T} \text { value } \\
(\mathrm{MPa} \sqrt{\mathrm{m}})\end{array}$ & \multirow{2}{*}{$\mathrm{T}_{\mathrm{oQ}}\left({ }^{\circ} \mathrm{C}\right)$} \\
\hline O002L* $^{*}$ & -167 & 25.0 & 22.5 & $\mathrm{~N}$ & $\mathrm{NA}$ & \\
\hline O002LM* $^{*}$ & -165 & 21.6 & 20.8 & $\mathrm{~N}$ & $\mathrm{NA}$ \\
\hline O002RM* $^{*}$ & -165 & 37.2 & 28.7 & $\mathrm{~N}$ & $\mathrm{NA}$ \\
\hline O002R* & -169 & 32.8 & 26.5 & $\mathrm{~N}$ & $\mathrm{NA}$ \\
\hline O005L & -132 & 62.5 & 41.4 & $\mathrm{~N}$ & $\mathrm{NA}$ \\
\hline O005LM & -126 & 243.3 & 132.6 & $\mathrm{Y}$ & 61.9 \\
\hline O005RM & -130 & 42.0 & 31.1 & $\mathrm{~N}$ & $\mathrm{NA}$ \\
\hline O003L & -129 & 104.9 & 62.8 & $\mathrm{Y}$ & 59.7 & \multirow{2}{*}{-79} \\
\hline O003LM & -129 & 169.3 & 95.3 & $\mathrm{Y}$ & 59.7 \\
\hline O003RM & -129 & 70.2 & 45.3 & $\mathrm{~N}$ & $\mathrm{NA}$ \\
\hline O003R & -128 & 98.8 & 59.7 & $\mathrm{Y}$ & 59.5 \\
\hline O004L & -132 & 98.7 & 59.7 & $\mathrm{~N}$ & $\mathrm{NA}$ \\
\hline O004LM & -130 & 35.6 & 27.9 & $\mathrm{~N}$ & $\mathrm{NA}$ \\
\hline O004RM & -131 & 46.2 & 33.2 & $\mathrm{~N}$ & $\mathrm{NA}$ \\
\hline O004R & -127 & 161.2 & 91.2 & $\mathrm{Y}$ & 60.5 & \\
\hline
\end{tabular}

*Fracture toughness results for information only, not used for Master Curve calculation

Table 15. Fracture toughness of Eurofer-97 Group P in the as-received condition

\begin{tabular}{|c|c|c|c|c|c|c|}
\hline $\begin{array}{l}\text { Specimen } \\
\text { ID }\end{array}$ & $\begin{array}{c}\text { Test } \\
\text { temperature } \\
\left({ }^{\circ} \mathrm{C}\right) \\
\end{array}$ & $\begin{array}{c}\mathrm{K}_{\mathrm{Jc}} \\
(\mathrm{MPa} \sqrt{\mathrm{m}})\end{array}$ & $\begin{array}{c}1 \mathrm{~T}-\mathrm{K}_{\mathrm{Jc}} \\
(\mathrm{MPa} \sqrt{\mathrm{m}})\end{array}$ & $\begin{array}{c}\text { Censored } \\
(\mathrm{Y} / \mathrm{N})\end{array}$ & $\begin{array}{l}\text { Censored } \\
1 \mathrm{~T} \text { value } \\
(\mathrm{MPa} \sqrt{\mathrm{m}})\end{array}$ & $\mathrm{T}_{\mathrm{oQ}}\left({ }^{\circ} \mathrm{C}\right)$ \\
\hline P002L* & -165 & 34.7 & 27.4 & $\mathrm{~N}$ & NA & \multirow{13}{*}{-87} \\
\hline P002LM* & -165 & 31.9 & 26.0 & $\mathrm{~N}$ & NA & \\
\hline P002RM* & -165 & 39.1 & 29.6 & $\mathrm{~N}$ & NA & \\
\hline P002R* & -165 & 34.5 & 27.3 & $\mathrm{~N}$ & NA & \\
\hline P003L & -132 & 239.3 & 130.6 & $\mathrm{Y}$ & 53.2 & \\
\hline P003LM & -131 & 298.9 & 160.6 & $\mathrm{Y}$ & 53.2 & \\
\hline P003RM & -131 & 312.4 & 167.4 & $\mathrm{Y}$ & 53.2 & \\
\hline P003R & -131 & 66.4 & 43.4 & $\mathrm{~N}$ & NA & \\
\hline P004L & -146 & 106.1 & 63.4 & $\mathrm{Y}$ & 58.7 & \\
\hline P004LM & -146 & 133.6 & 77.3 & $\mathrm{Y}$ & 58.6 & \\
\hline P004RM & -145 & 76.1 & 48.3 & $\mathrm{~N}$ & NA & \\
\hline P004R & -147 & 69.9 & 45.2 & $\mathrm{~N}$ & NA & \\
\hline P005L & -146 & 85.9 & 53.2 & $\mathrm{~N}$ & NA & \\
\hline
\end{tabular}




\begin{tabular}{|c|c|c|c|c|c|}
\hline P005LM & -146 & 266.9 & 144.5 & $\mathrm{Y}$ & 53.2 \\
\hline P005RM & -146 & 44.8 & 32.5 & $\mathrm{~N}$ & NA \\
\hline P005R & -146 & 74.4 & 47.4 & $\mathrm{~N}$ & NA \\
\hline
\end{tabular}

*Fracture toughness results for information only, not used for Master Curve calculation

Table 16. Fracture toughness of Eurofer-97 Group E in the as-received condition

\begin{tabular}{|c|c|c|c|c|c|c|}
\hline $\begin{array}{l}\text { Specimen } \\
\text { ID }\end{array}$ & $\begin{array}{c}\text { Test } \\
\text { temperature } \\
\left({ }^{\circ} \mathrm{C}\right)\end{array}$ & $\begin{array}{c}\mathrm{K}_{\mathrm{Jc}} \\
(\mathrm{MPa} \sqrt{\mathrm{m}})\end{array}$ & $\begin{array}{c}1 \mathrm{~T}-\mathrm{K}_{\mathrm{Jc}} \\
(\mathrm{MPa} \sqrt{\mathrm{m}})\end{array}$ & $\begin{array}{c}\text { Censored } \\
(\mathrm{Y} / \mathrm{N})\end{array}$ & $\begin{array}{l}\text { Censored } \\
1 \mathrm{~T} \text { value } \\
(\mathrm{MPa} \sqrt{\mathrm{m}})\end{array}$ & $\mathrm{T}_{\mathrm{oQ}}\left({ }^{\circ} \mathrm{C}\right)$ \\
\hline E032L* & -112 & 39.7 & 29.9 & $\mathrm{~N}$ & NA & \multirow{21}{*}{-29} \\
\hline E032LM* & -112 & 52.5 & 36.4 & $\mathrm{~N}$ & $\mathrm{NA}$ & \\
\hline E032RM* & -169 & 20.2 & 20.1 & $\mathrm{~N}$ & $\mathrm{NA}$ & \\
\hline E032R* & -169 & 18.1 & 19.0 & $\mathrm{~N}$ & $\mathrm{NA}$ & \\
\hline E035L* & -112 & 50.7 & 35.5 & $\mathrm{~N}$ & $\mathrm{NA}$ & \\
\hline E035LM* & -112 & 33.1 & 26.6 & $\mathrm{~N}$ & NA & \\
\hline E035RM* & -166 & 19.7 & 19.8 & $\mathrm{~N}$ & $\mathrm{NA}$ & \\
\hline E035R* & -169 & 23.3 & 21.7 & $\mathrm{~N}$ & NA & \\
\hline E031RM & -90 & 73.9 & 47.2 & $\mathrm{~N}$ & NA & \\
\hline E031R & -90 & 82.4 & 51.5 & $\mathrm{~N}$ & NA & \\
\hline E034L & -90 & 132.0 & 76.5 & $\mathrm{Y}$ & 53.5 & \\
\hline E034LM & -90 & 72.3 & 46.4 & $\mathrm{~N}$ & NA & \\
\hline E034RM & -90 & 87.3 & 53.9 & $\mathrm{Y}$ & 52.8 & \\
\hline E034R & -90 & 71.1 & 45.8 & $\mathrm{~N}$ & NA & \\
\hline E033L & -90 & 100.0 & 60.3 & $\mathrm{Y}$ & 53.2 & \\
\hline E033LM & -90 & 104.1 & 62.4 & $\mathrm{Y}$ & 52.8 & \\
\hline E033R & -90 & 90.77 & 55.7 & $\mathrm{Y}$ & 53.2 & \\
\hline E025L & -89 & 68.1 & 44.2 & $\mathrm{~N}$ & NA & \\
\hline E025LM & -92 & 51.8 & 36.0 & $\mathrm{~N}$ & NA & \\
\hline E025RM & -91 & 68.3 & 44.4 & $\mathrm{~N}$ & NA & \\
\hline E025R & -90 & 102.1 & 61.4 & $Y$ & 53.2 & \\
\hline
\end{tabular}

*Fracture toughness results for information only, not used for Master Curve calculation

Table 17. Fracture toughness of Eurofer-97 Group E in the heat-treated condition

\begin{tabular}{|c|c|c|c|c|c|c|}
\hline $\begin{array}{l}\text { Specimen } \\
\text { ID }\end{array}$ & $\begin{array}{c}\text { Test } \\
\text { temperature } \\
\left({ }^{\circ} \mathrm{C}\right)\end{array}$ & $\begin{array}{c}\mathrm{K}_{\mathrm{Jc}} \\
(\mathrm{MPa} \sqrt{ } \mathrm{m})\end{array}$ & $\begin{array}{c}1 \mathrm{~T}-\mathrm{K}_{\mathrm{Jc}} \\
(\mathrm{MPa} \sqrt{\mathrm{m}})\end{array}$ & $\begin{array}{c}\text { Censored } \\
(\mathrm{Y} / \mathrm{N})\end{array}$ & $\begin{array}{l}\text { Censored } \\
1 \mathrm{~T} \text { value } \\
(\mathrm{MPa} \sqrt{\mathrm{m}}) \\
\end{array}$ & $\mathrm{T}_{\mathrm{oQ}}\left({ }^{\circ} \mathrm{C}\right)$ \\
\hline $1 \mathrm{~L}$ & -159 & 76.6 & 48.5 & $\mathrm{~N}$ & NA & \multirow{15}{*}{-92} \\
\hline $1 \mathrm{LM}$ & -159 & 90.8 & 55.7 & $\mathrm{~N}$ & NA & \\
\hline $1 \mathrm{RM}$ & -159 & 78.3 & 49.4 & $\mathrm{~N}$ & NA & \\
\hline $1 \mathrm{R}$ & -159 & 53.1 & 36.7 & $\mathrm{~N}$ & NA & \\
\hline $2 \mathrm{~L}$ & -153 & 256.2 & 139.1 & $\mathrm{Y}$ & 55.7 & \\
\hline $2 \mathrm{LM}$ & -156 & 63.1 & 41.7 & $\mathrm{~N}$ & NA & \\
\hline $2 \mathrm{RM}$ & -151 & 326.0 & 174.3 & $\mathrm{Y}$ & 55.7 & \\
\hline $2 \mathrm{R}$ & -153 & 77.6 & 49.0 & $\mathrm{~N}$ & NA & \\
\hline $3 \mathrm{~L}$ & -158 & 82.0 & 51.3 & $\mathrm{~N}$ & NA & \\
\hline $3 \mathrm{LM}$ & -157 & 108.0 & 64.4 & $\mathrm{Y}$ & 63.0 & \\
\hline $3 \mathrm{RM}$ & -159 & 32.5 & 26.3 & $\mathrm{~N}$ & NA & \\
\hline $4 \mathrm{~L}$ & -152 & 288.5 & 155.4 & $\mathrm{Y}$ & 55.7 & \\
\hline $4 \mathrm{LM}$ & -152 & 86.1 & 53.3 & $\mathrm{~N}$ & NA & \\
\hline $4 \mathrm{RM}$ & -157 & 48.4 & 34.3 & $\mathrm{~N}$ & NA & \\
\hline $4 \mathrm{R}$ & -157 & 39.8 & 30.0 & $\mathrm{~N}$ & NA & \\
\hline
\end{tabular}




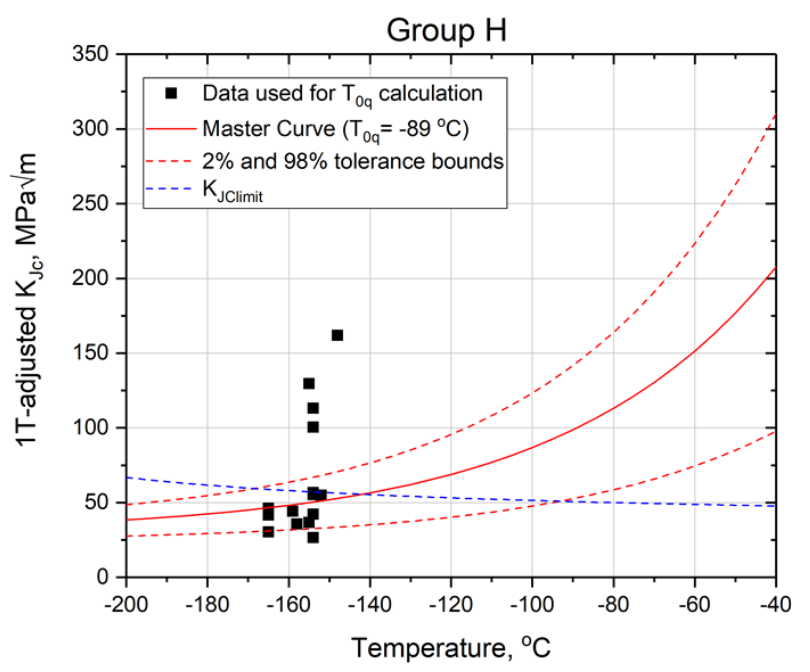

Fig. 8. Master Curve results of Eurofer-97 Group H in the as-received condition

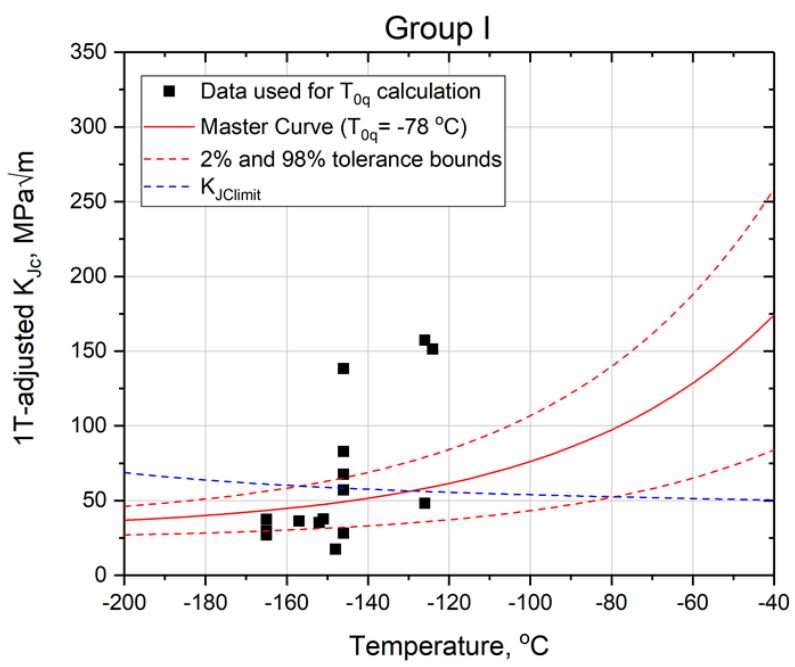

Fig. 9. Master Curve results of Eurofer-97 Group I in the as-received condition

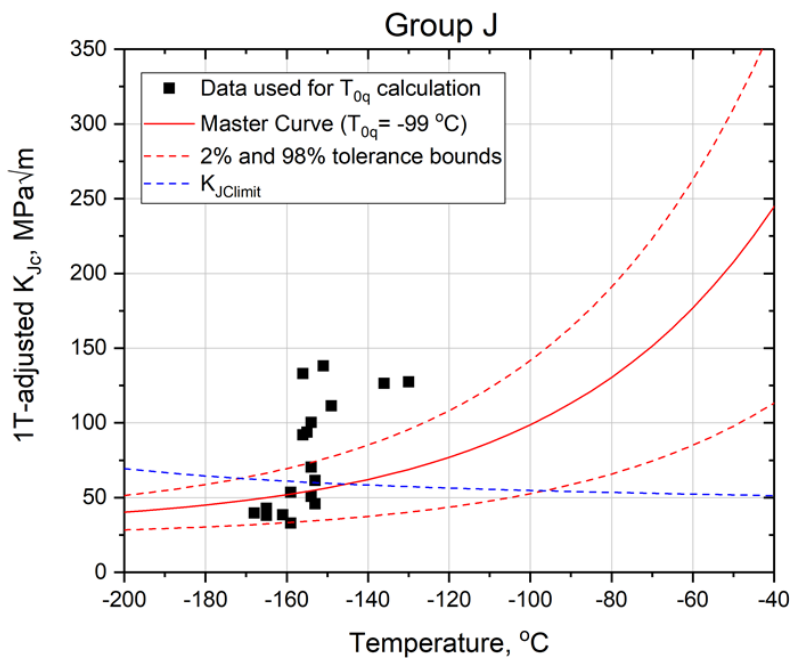

Fig. 10. Master Curve results of Eurofer-97 Group J in the as-received condition 


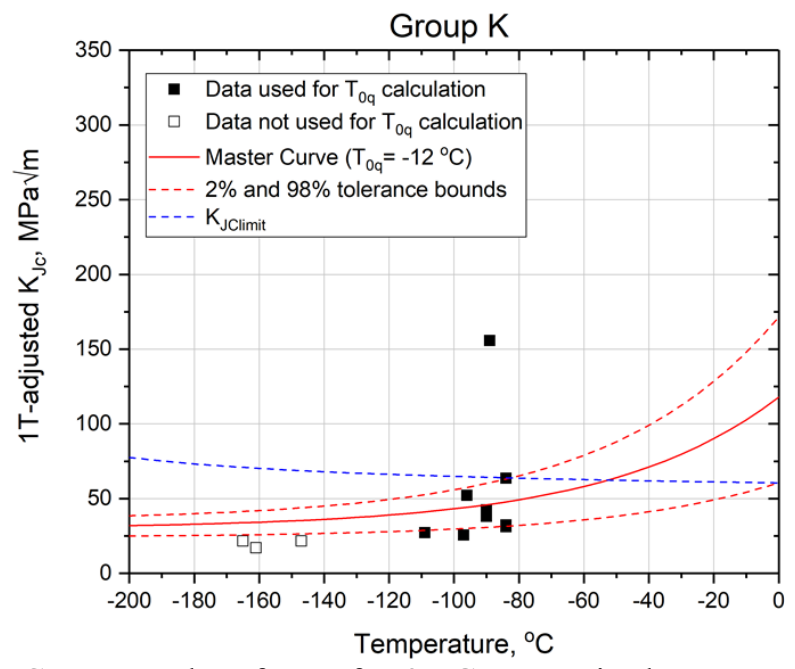

Fig. 11. Master Curve results of Eurofer-97 Group K in the as-received condition

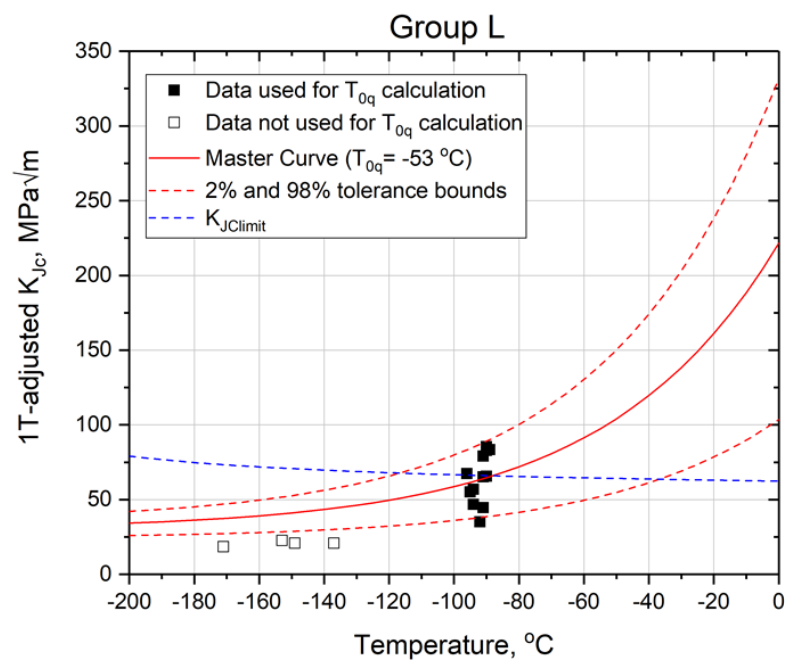

Fig. 12. Master Curve results of Eurofer-97 Group L in the as-received condition

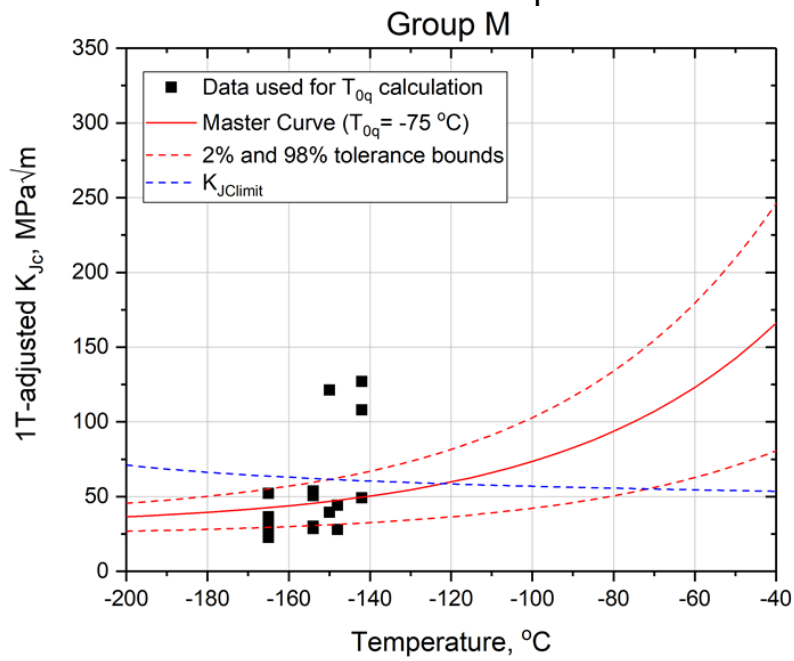

Fig. 13. Master Curve results of Eurofer-97 Group $\mathrm{M}$ in the as-received condition 


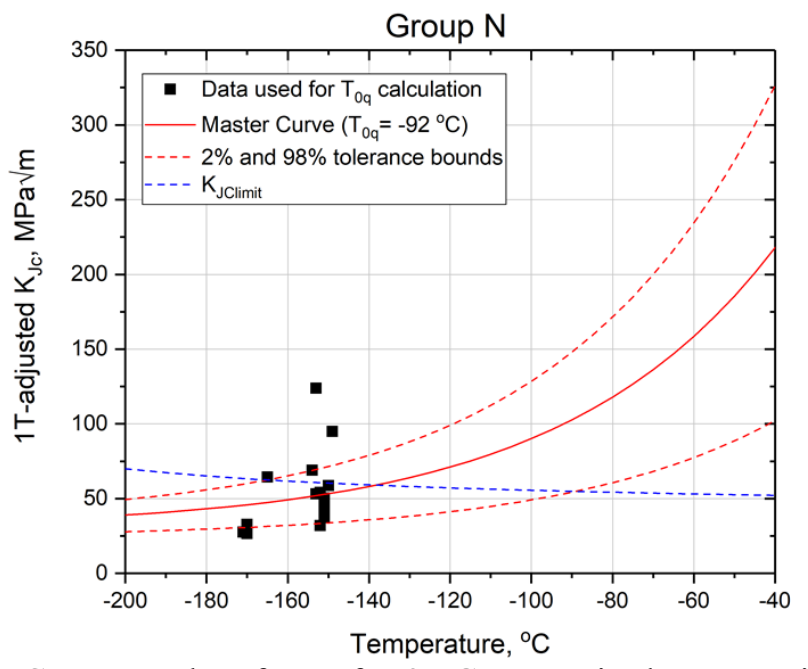

Fig. 14. Master Curve results of Eurofer-97 Group $\mathrm{N}$ in the as-received condition

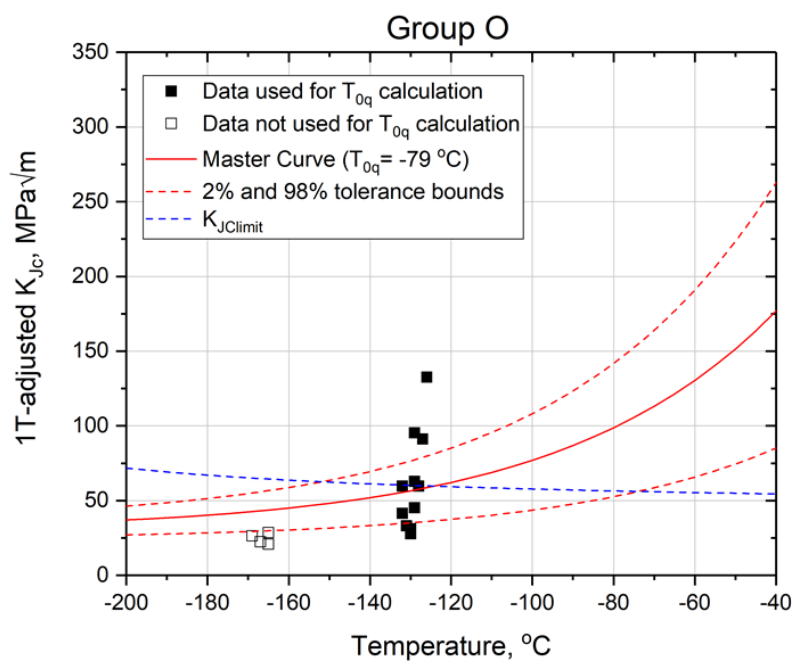

Fig. 15. Master Curve results of Eurofer-97 Group O in the as-received condition

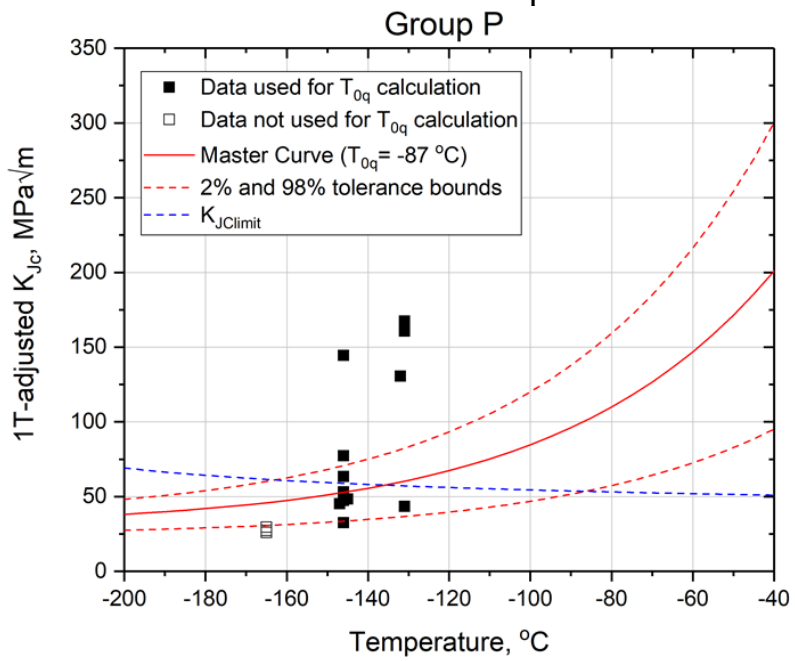

Fig. 16. Master Curve results of Eurofer-97 Group P in the as-received condition 


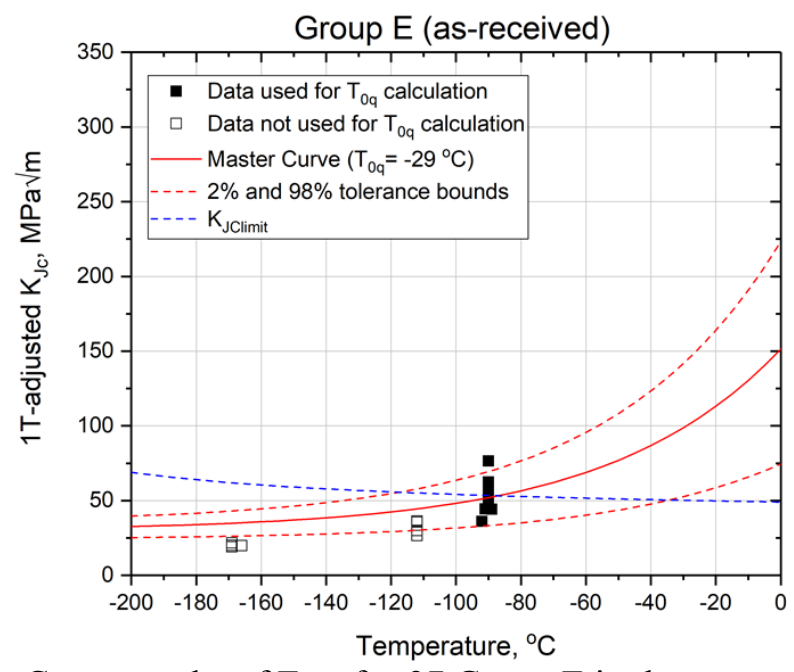

Fig. 17. Master Curve results of Eurofer-97 Group E in the as-received condition

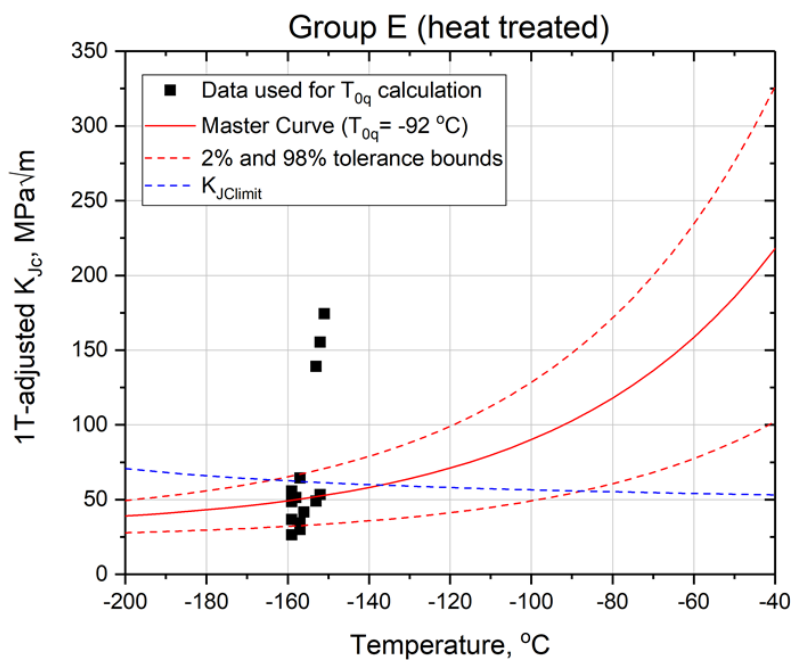

Fig. 18. Master Curve results of Eurofer-97 Group E in in the heat-treated condition

\subsection{Microstructure}

\subsubsection{L series steel: CEA}

Light optical microscopy (LOM) images of the L series steel etched using Villela's reagent are shown in Fig. 19, which revealed a typical tempered martensitic structure consisting of martensitic laths and prior austenite grains (PAGs). It is evident from the figure that PAG size was very large in this steel, exceeding $50 \mu \mathrm{m}$. Large PAGs are expected due to the very high normalizing temperature of $1150{ }^{\circ} \mathrm{C}$ as opposed to $980{ }^{\circ} \mathrm{C}$ conventionally used for $9 \% \mathrm{Cr}$ F-M steels [3]. The imaging was performed along the rolling direction on the RD-ND plane. A typical etched tempered martensitic structure was also evident from the SEM secondary electron image (Fig. 20). No inclusions were detected in the steel, neither by LOM nor by SEM.

SEM analysis was also performed to study the fracture surface after tensile tests, which revealed a mixed mode of fracture in this steel. Majority of the sample surface failed in a ductile fashion, but numerous areas showed cleavage fracture. Fig. 21a shows the fracture surface overview where some areas showing brittle cleavages are indicated using arrows. Similar brittle areas are depicted in Figs. 21b and 21d at relatively high magnifications. Judging by the way the two cleavage planes are separating in the brittle 
areas, it appears that these cracks maybe running preferentially along the large PAGs of this steel. As will be shown in the ensuing subsections, similar fracture behaviour was noted in $\mathrm{K}$ series steel, which also consisted of very large PAGs. The regions away from the brittle cleavages showed dimpled fracture typical of ductile materials, but showing cup and cone fracture expected due to presence of carbides/nitrides in RAFM steels (Fig. 21c). No excessively large dimples, suggestive of inclusions, were detected on the fracture surface.
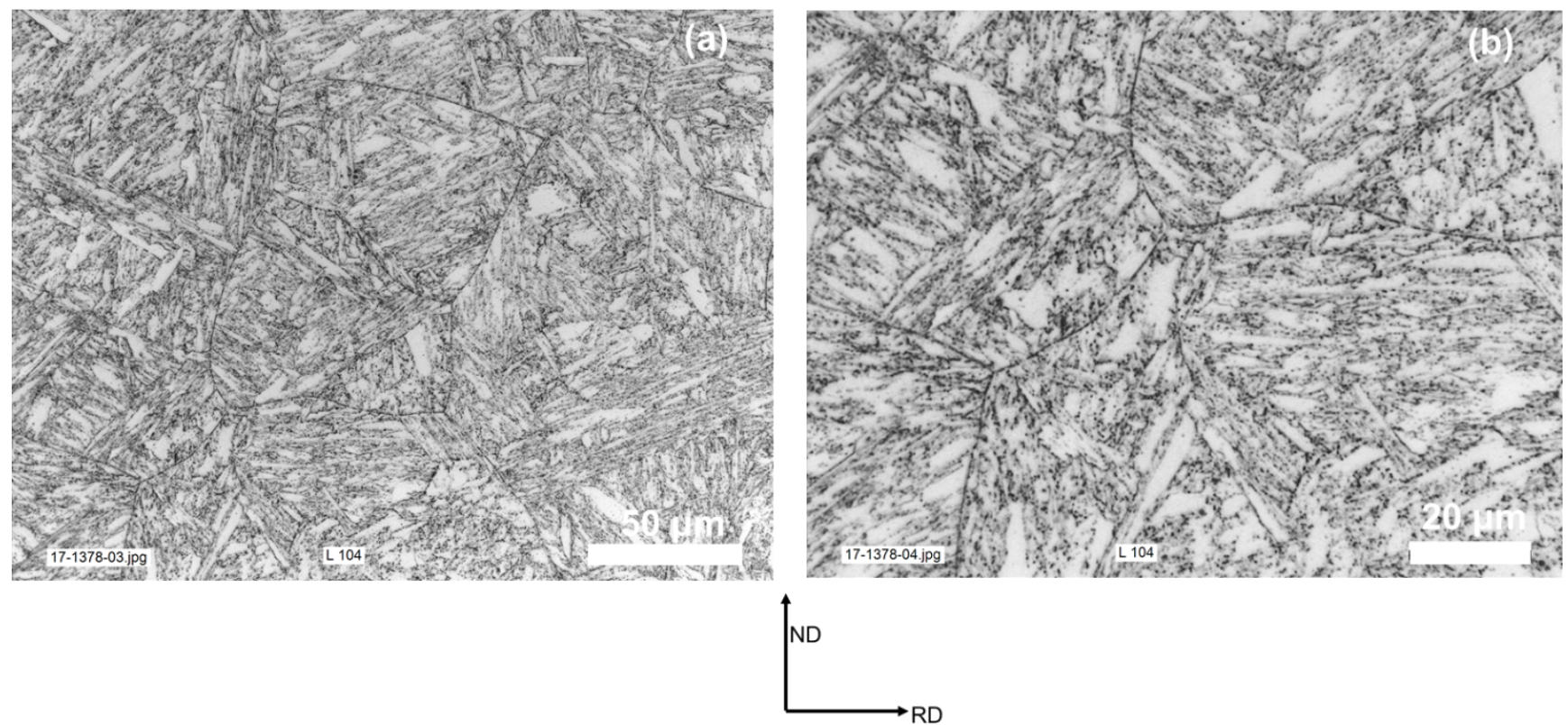

Fig. 19. LOM images at two different magnifications of L series steel after etching using Villela's reagent. $\mathrm{RD}=$ rolling direction, $\mathrm{ND}=$ normal direction .

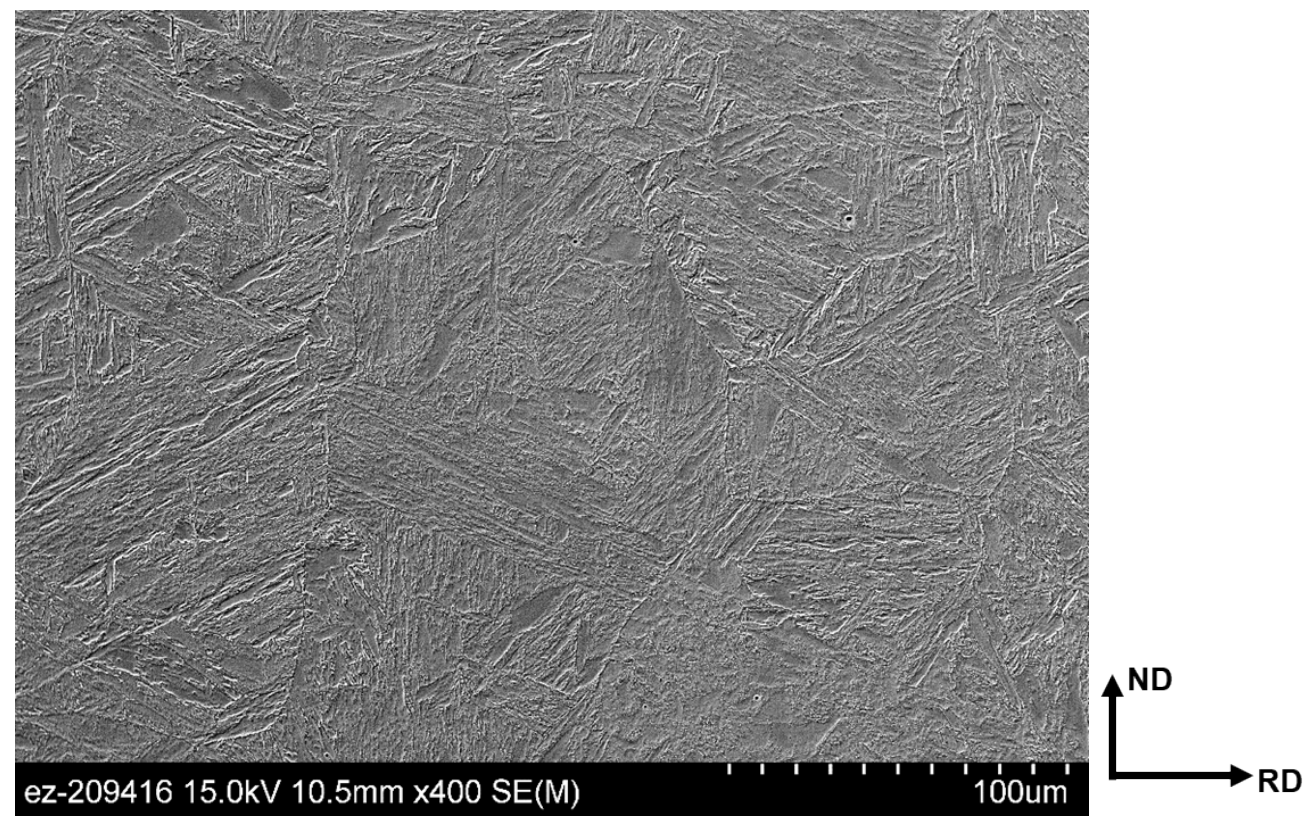

Fig. 20. SEM secondary electron image of L series steel in etched condition. 

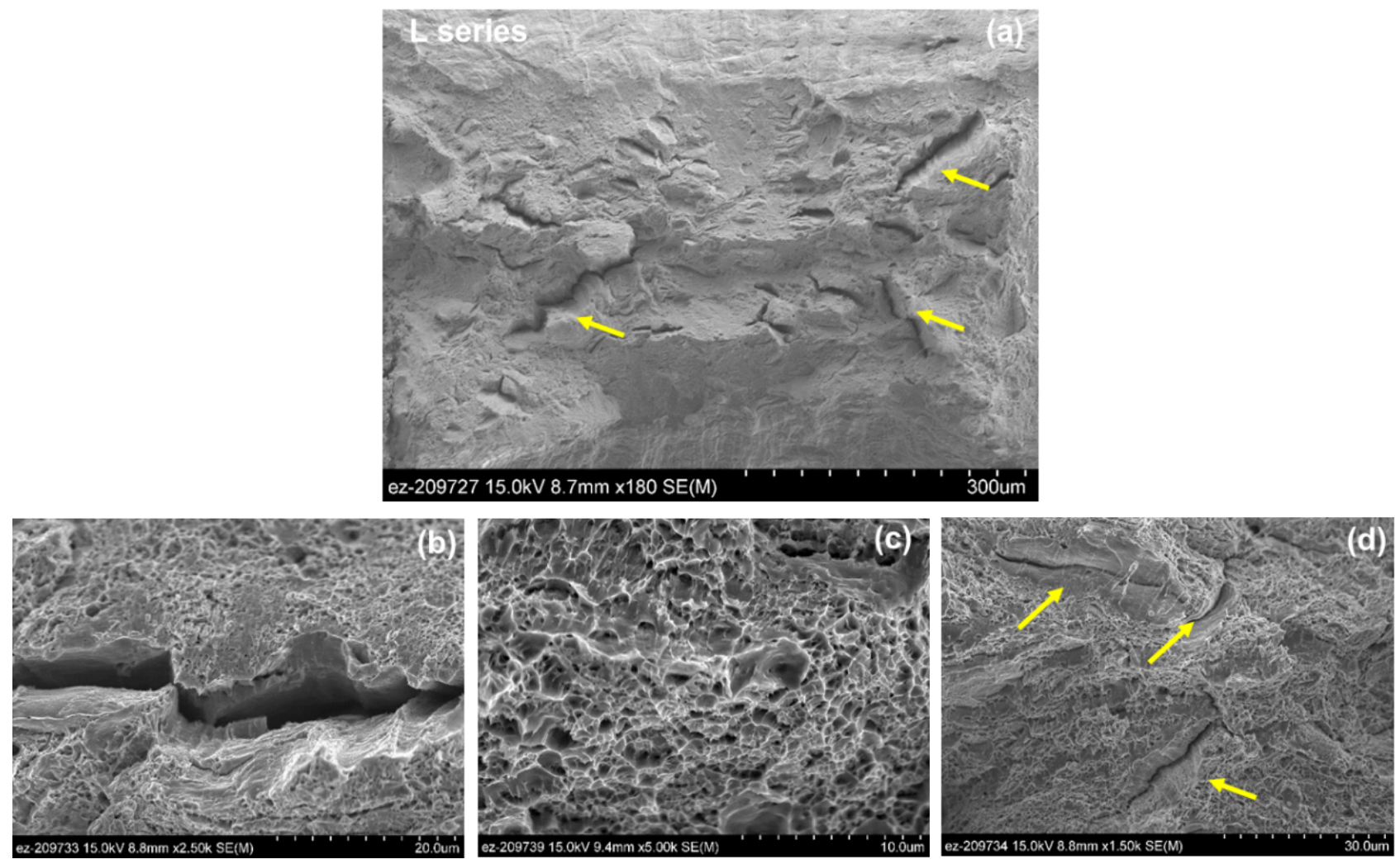

Fig. 21. SEM secondary electron imaging of fracture surface of $L$ series steel. (a) Fracture surface overview. (b, c, d) Relatively high magnification images of different areas of the fracture surface showing brittle cleavages and ductile dimpled areas.

STEM imaging was performed mainly to see the precipitation behavior in this steel. These experiments were performed on FIB specimens extracted from the same samples on which tensile tests were conducted, from the head/grip sections. Figs. 22a-22c show BF and medium angle annular dark field (MAADF-DF2) images of the sample showing the typical lath structure. Large carbides were detected along the lath boundaries, indicated using arrows in Figs. $22 \mathrm{~b}-22 \mathrm{c}$, which are expected to be the $\mathrm{M}_{23} \mathrm{C}_{6}$ type. Some small precipitates were also detected. These precipitates were slightly better visible in the MAADF-DF2 images compared to BF. Henry et al. [4] has shown previously that this steel mainly consisted of Cr, $\mathrm{V}$ rich $\mathrm{M}_{2} \mathrm{X}$ nitride phase instead of MX carbo-nitrides. Therefore, the smaller precipitates are expected to be mainly $\mathrm{M}_{2} \mathrm{X}$. Fig. 22c was imaged close to two beam conditions which brought the high dislocation density of the laths come into contrast. Fig. 22d shows a few 5-7 nm size precipitates detected in the steel which can be either $\mathrm{M}_{2} \mathrm{X}$ or MX.

It is important to reiterate that due to a similar $\mathrm{Z}$ of precipitate constituents compared to matrix, and high dislocation density of the tempered martensitic structure, carbides/nitrides are not well revealed using conventional TEM and STEM images. It is a typical issue in characterizing F-M steels. An example of this can be seen in Fig. 22c where an indicated $\mathrm{M}_{23} \mathrm{C}_{6}$ phase appears rather faint, despite having a large size $(>100 \mathrm{~nm})$. The situation is more complex for smaller precipitates. Therefore, performing TEM/STEM imaging is not sufficient to develop an understanding of the microstructure. However, imaging precipitates can be greatly improved if high count rate EDX mapping is performed using latest state-of-art ChemiSTEM systems. For this reason, we performed EDX mapping in STEM mode in a FEI F200X Talos to reveal the precipitates. The advantage is that using EDX, only precipitates are detected and not the high dislocation density. Further, EDX mapping is also largely sufficient to unambiguously differentiate between 
$\mathrm{M}_{23} \mathrm{C}_{6}$ and $\mathrm{MX}$ phases because the latter are mostly highly enriched in Ta, $\mathrm{V}$ and $\mathrm{N}$ while $\mathrm{M}_{23} \mathrm{C}_{6}$ typically consists of all the carbide formers. Using this approach, EDX maps revealing the precipitates in the steel are shown in Figs. 23, 24 and 25.
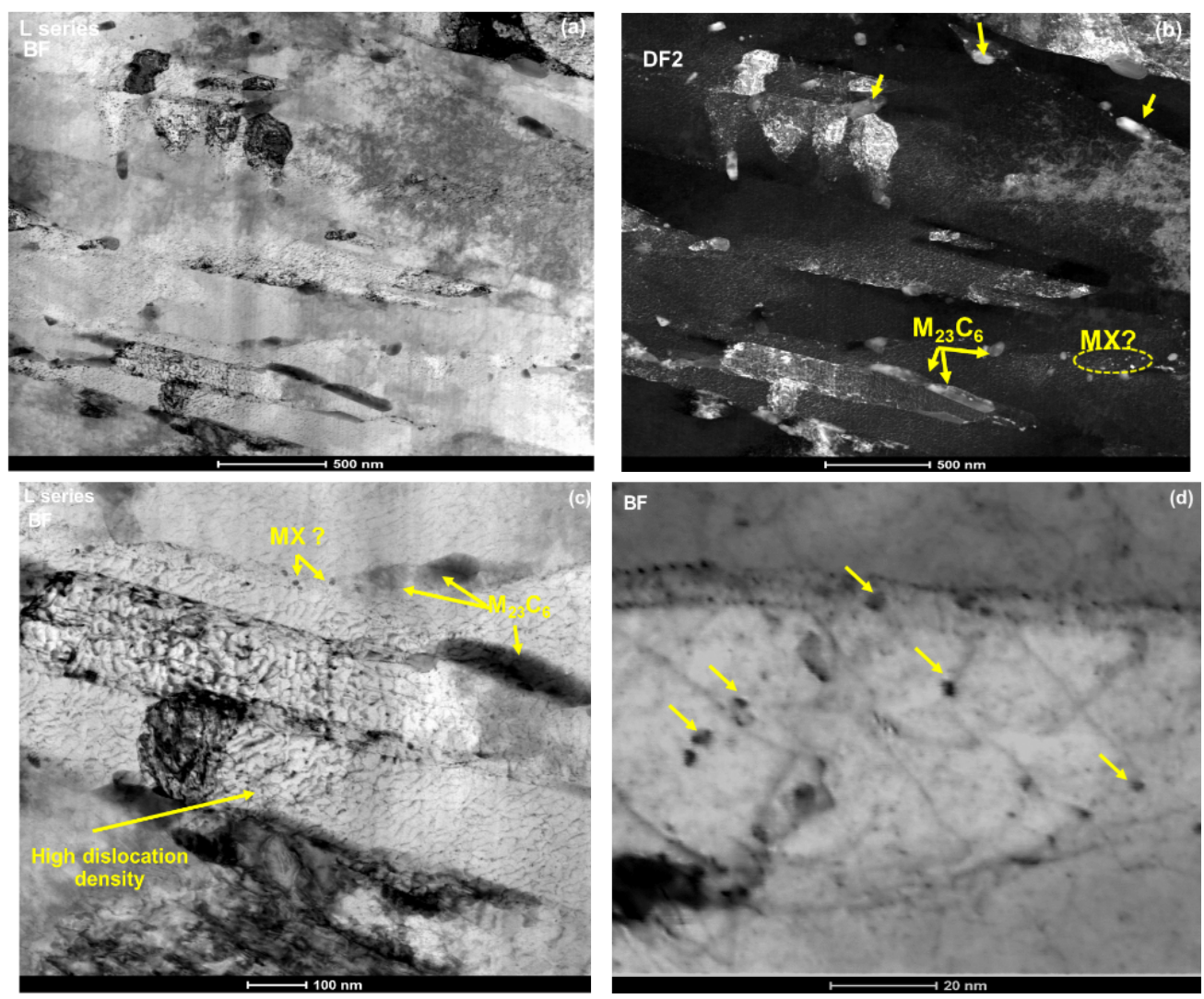

Fig. 22. STEM imaging of L series steel. $(a, b) B F$ and MAADF images showing the sample overview, with some precipitates indicated using arrows. (c) BF image close to two beam conditions showing the high dislocation density. (d) High magnification BF STEM image close to two-beam condition revealing
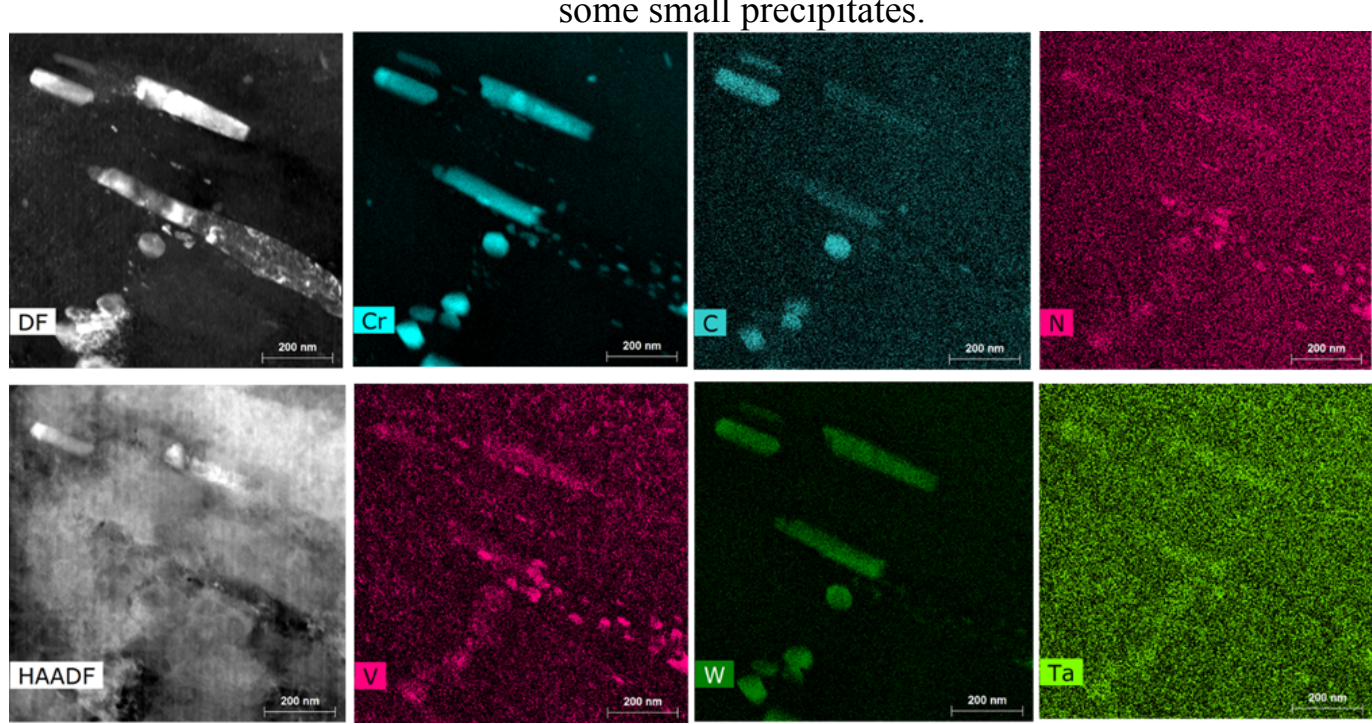

Fig. 23. STEM-EDX mapping of precipitates in L series steel, along with an MAADF (DF) and HAADF.

The larger elongated precipitates in Fig, 23 are expected to be $\mathrm{M}_{23} \mathrm{C}_{6}$ carbides and were mainly present along the lath boundaries. EDX also revealed small (less than $10 \mathrm{~nm}$ ) size precipitates which were predominantly $\mathrm{Cr}$ and $\mathrm{V}$ rich. These precipitates also generated a relatively strong nitrogen signal. We 
believe these are the $\mathrm{M}_{2} \mathrm{X}$ nitride precipitates as detected in the same steel by Henry et al. Two more examples of these small precipitates, imaged at relatively higher magnifications, is presented in Figs. 24 and 25. Once again, these precipitates were found to be mainly $\mathrm{Cr}$, $\mathrm{V}$ rich nitrides. Very few precipitates generating a very strong Ta signal were detected in all the EDX maps, indicating that little to no MX was detected in the samples. Fig. 25 maps were generated from a second FIB foil from the same L series batch.
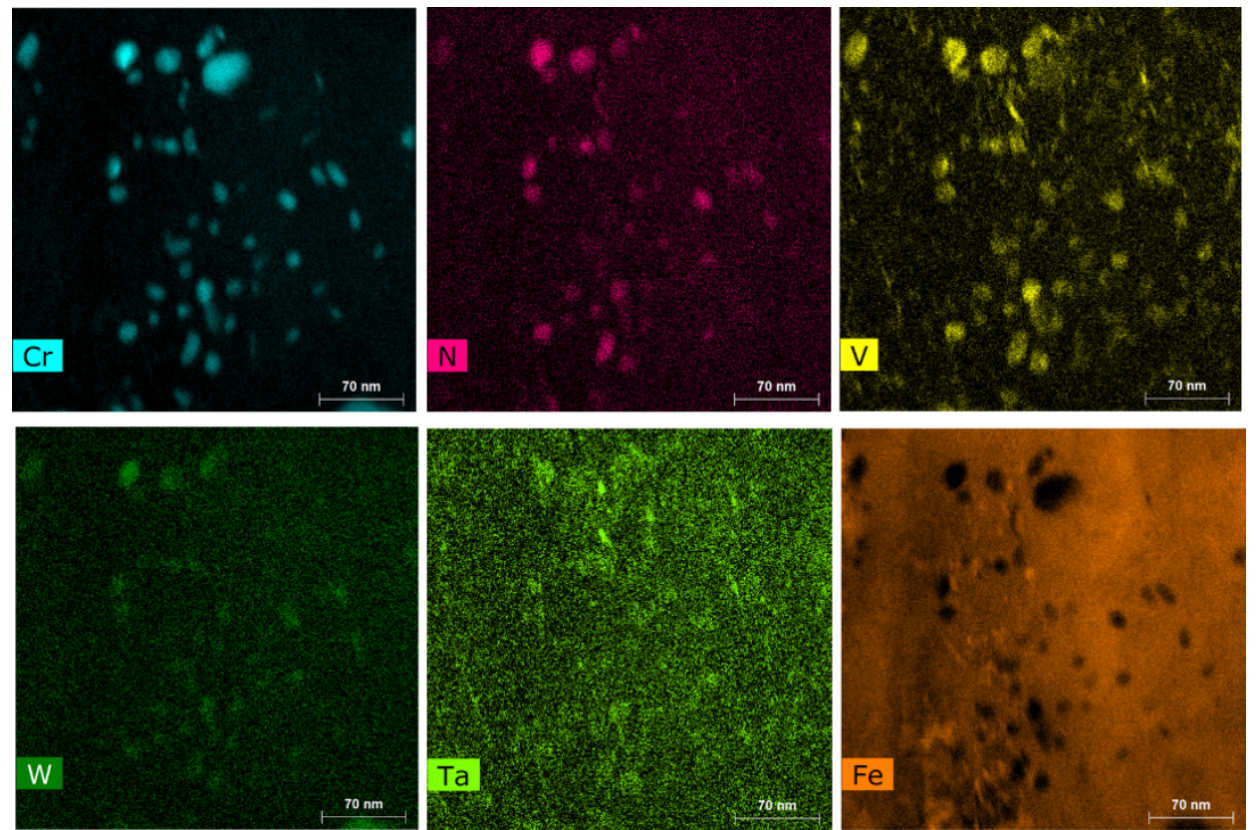

Fig. 24. High magnification STEM-EDX mapping of $\mathrm{Cr}-\mathrm{V}$ rich precipitates in L series steel.
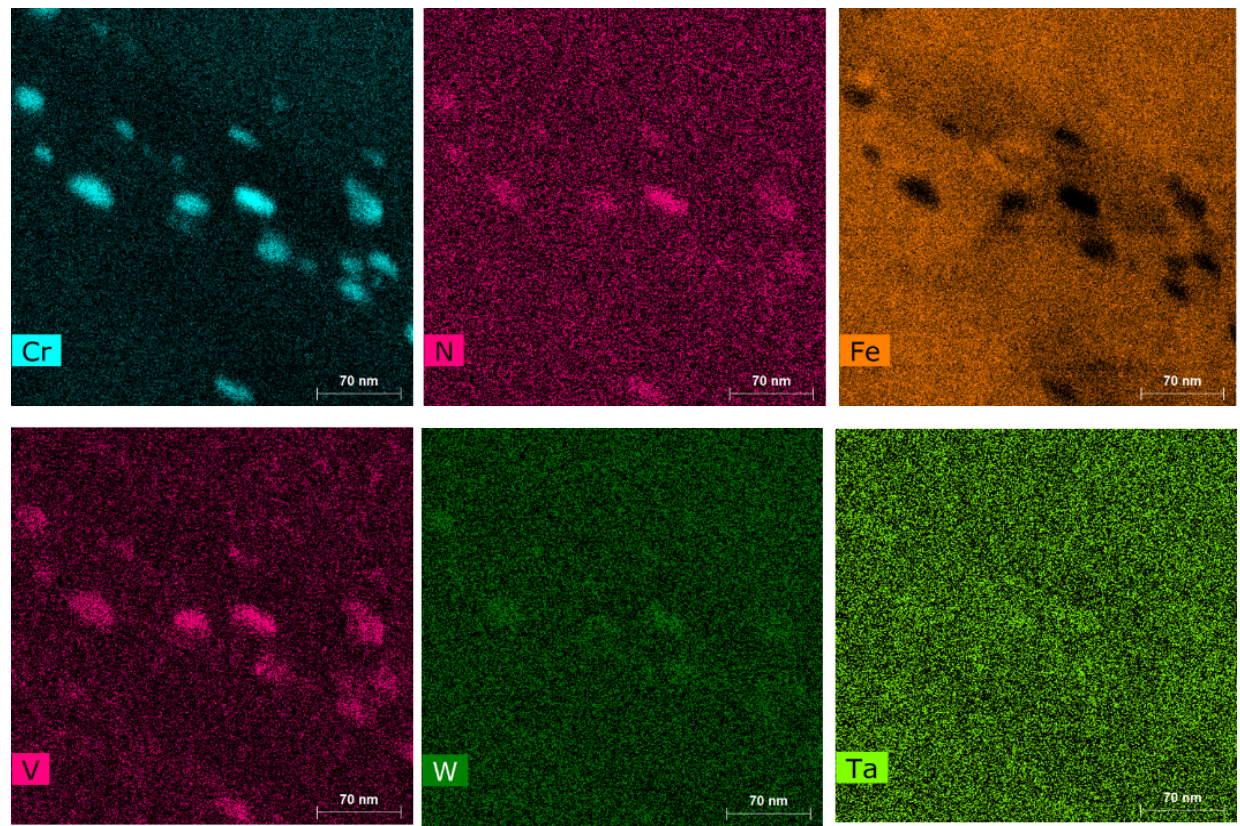

Fig. 25. High magnification STEM-EDX mapping of $\mathrm{Cr}-\mathrm{V}$ rich precipitates in $\mathrm{L}$ series steel (second FIB foil). 


\subsubsection{H, I and P series steels: KIT}

Fig. 26 presents the optical images of the etched microstructures of $\mathrm{H}$, I and P series steels. The imaging was performed on the RD-ND plane as indicated in the figure. Judging by these images, the microstructure seems over-tempered. $\mathrm{H}$ and I series steels contained ferrite grains. These grains appeared much brighter in the etched steels without much carbides (black dots). Some identified ferrite grains in these two steels are indicated using arrows. SEM BSE images showing the ferrite grains detected in $\mathrm{H}$ and I steels are also presented, in Fig. 27. P series steel did not seem to contain the ferrite grains. It is evident from Fig. 26 that $\mathrm{H}$ series steel had larger grains as compared to the I and P.
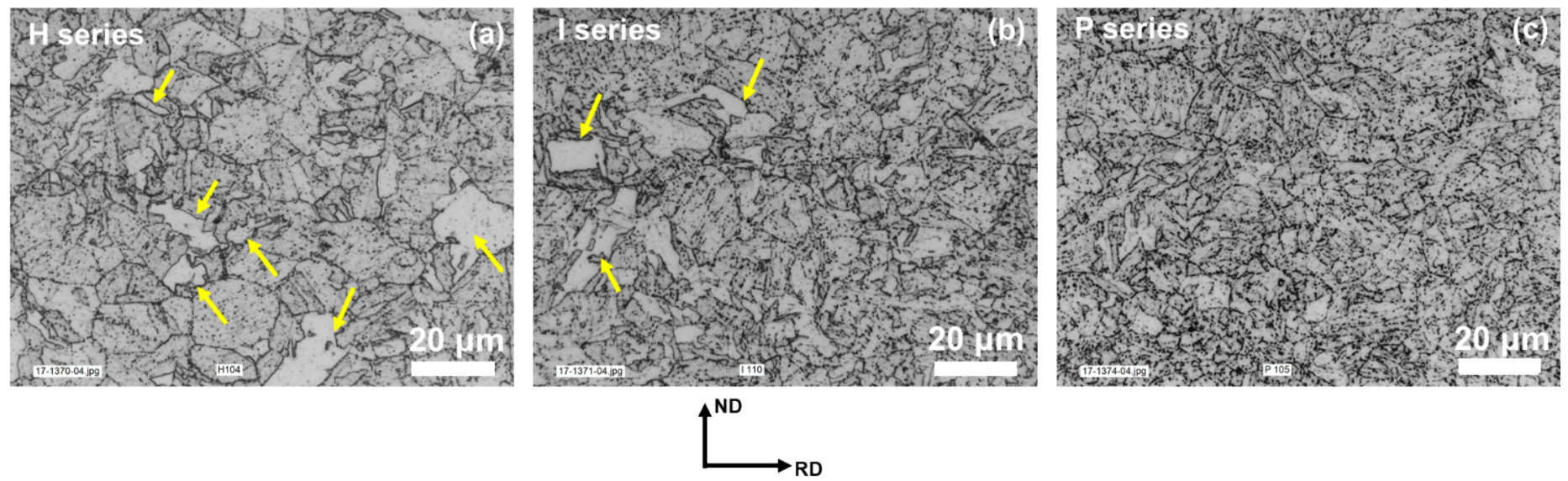

Fig. 26. LOM images of $\mathrm{H}$, I and $\mathrm{P}$ series steel after etching using Villela's reagent. Small arrows indicate the ferrite grains.
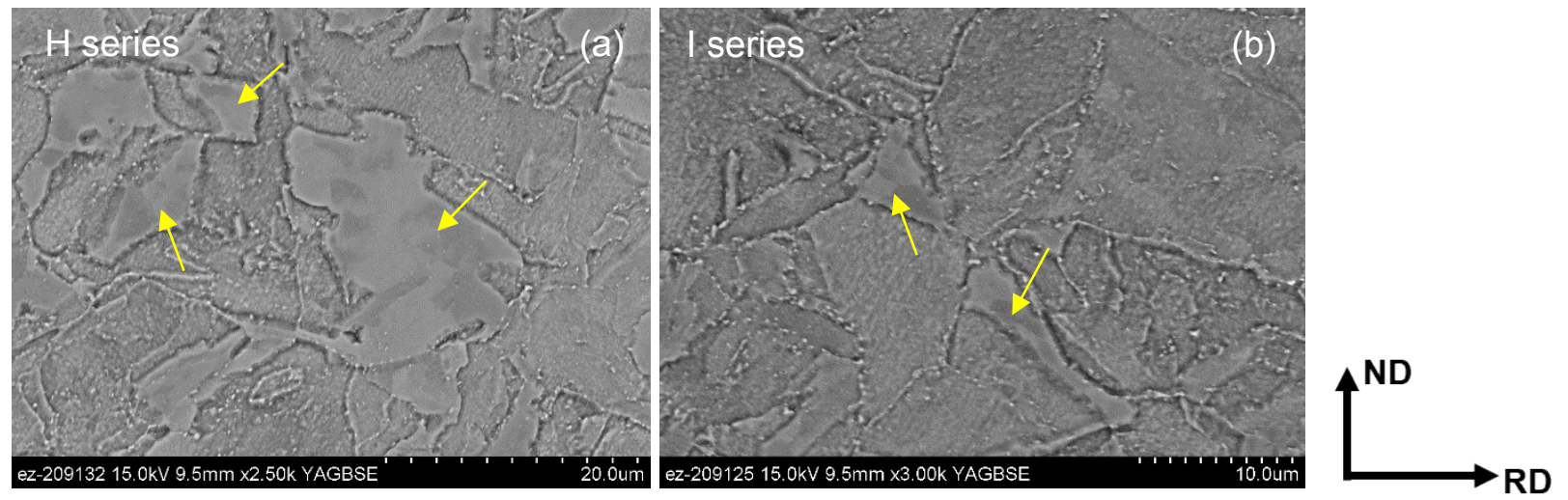

Fig. 27. SEM-BSE images showing the ferrite grains in $\mathrm{H}$ and I series steels.

SEM analysis was also performed to study the fracture surface after room temperature tensile tests of H, I and P steels. All the three steels showed a highly dimpled fracture surface, typical of ductile metals and containing cup and cone features expected due to the presence of carbides (Fig. 28). A ductile failure of these three steels is fully consistent with the tensile tests results. Additionally, the fracture surface showed many large holes, which suggest the presence of a low density of large $(>1 \mu \mathrm{m})$ inclusions in the steels. Such holes, or large cup and cone type of features on the fracture surface were particularly prominent for the I series steel (Fig. 28e). Relatively high magnification images of these fracture features are shown in Figs. 29a-29c where inclusion can be identified inside the burrows. Because these inclusions were embedded in the cones of the fracture, it was not possible to check their chemistry using EDX. However, a few large inclusions were detected in SEM analysis of the etched samples surfaces - see backscattered electron images (BSE) images in Figs. 29d-29f. Because these inclusions appeared bright in BSE images, 
they must contain heavy elements like Ta. We performed an EDX spot spectra in SEM from the inclusion in Fig. 29 e, where the inclusion showed a very high Ta signal (Fig.30). We believe, these are large tantalum oxide inclusion that are not uncommon in RAFM steels and they were responsible for the large cup and cone fracture features detected in the steel and were acting as fracture initiation sites.
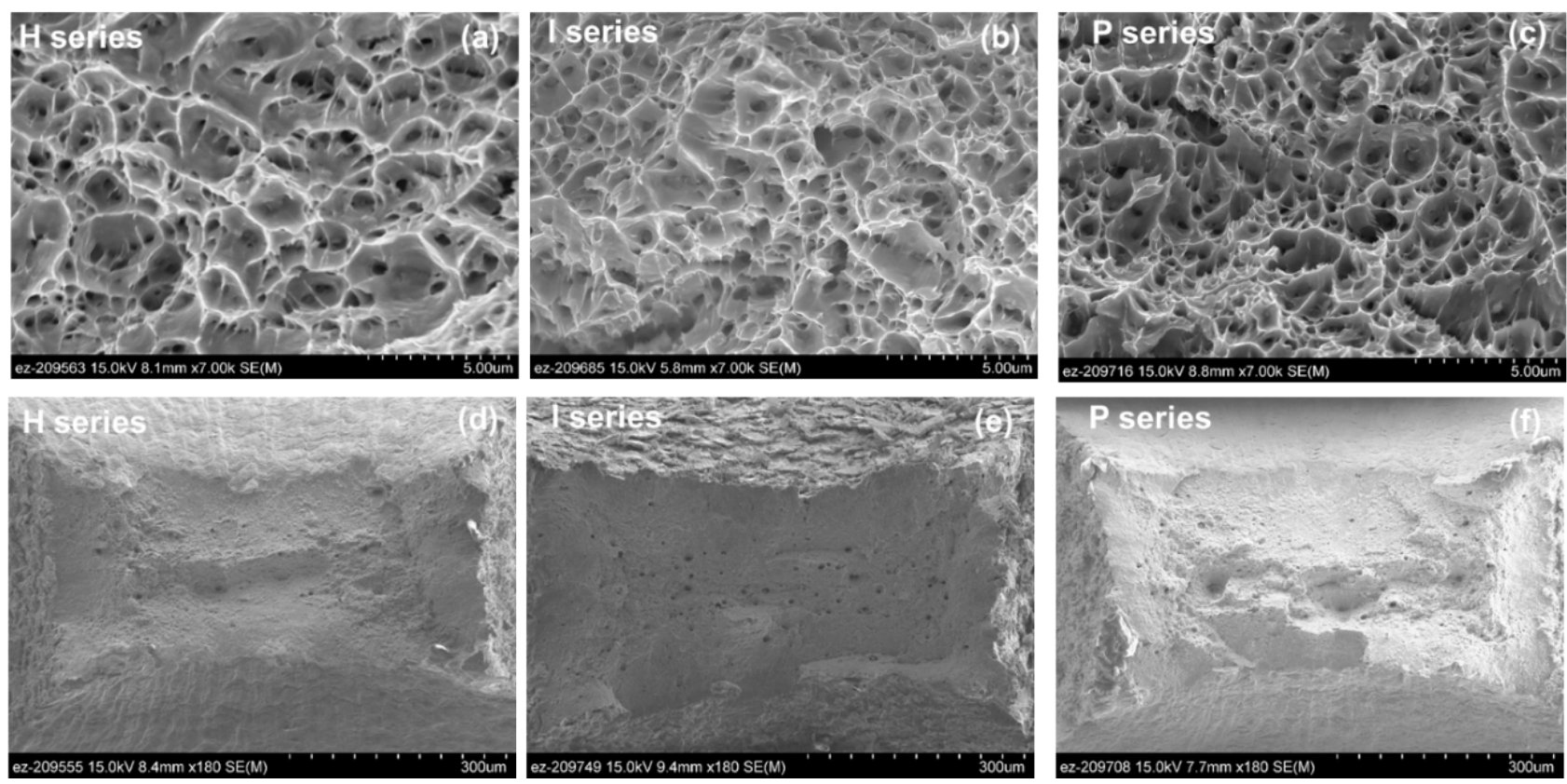

Fig. 28. SEM analysis of the fracture surface. $(a, b, c)$. High magnification secondary electron images. (d, e, f) Overview of the fracture surfaces of the three steels.
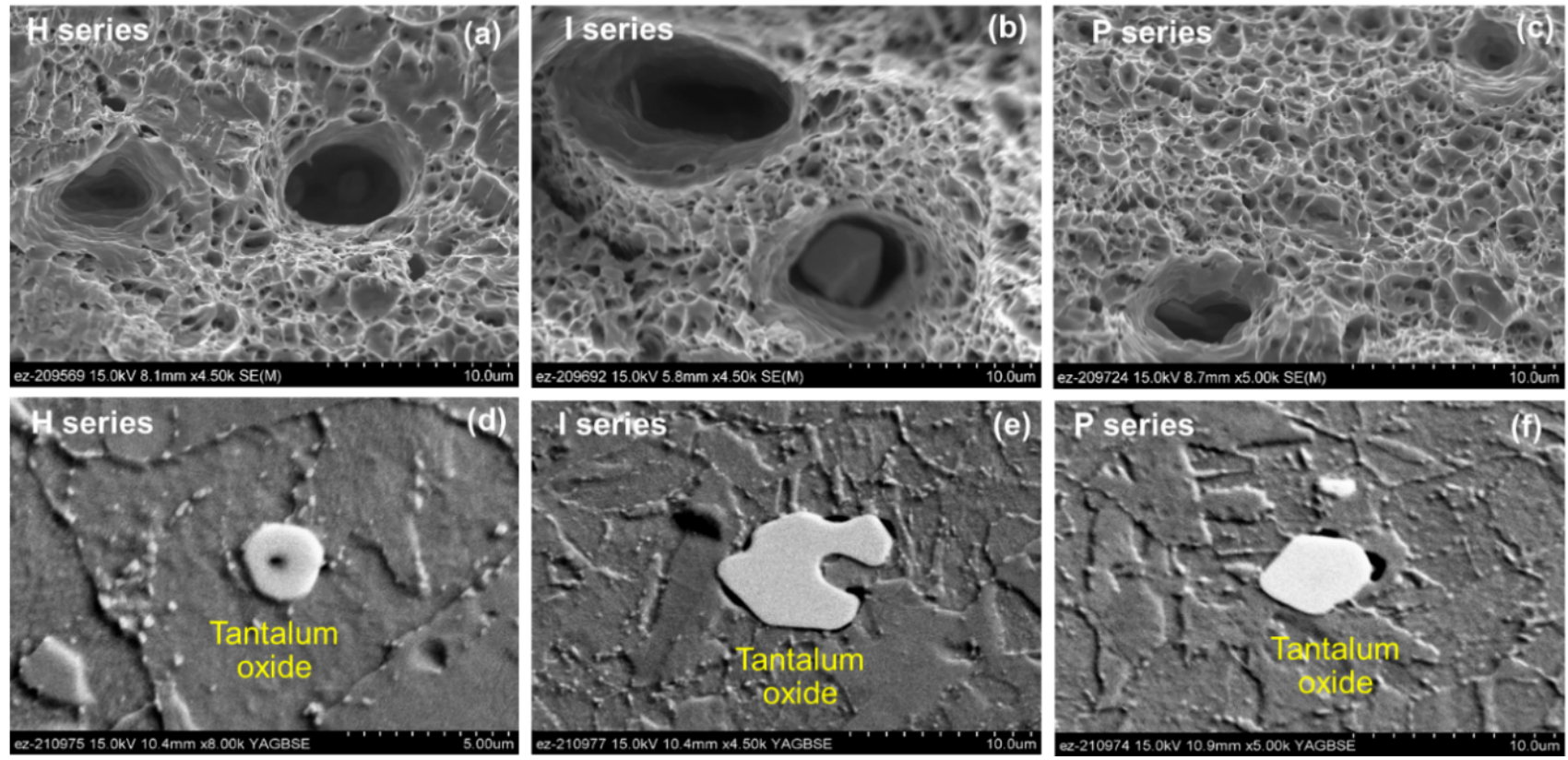

Fig. 29. SEM analysis showing large cup and cone fractures, and steel inclusions. (a, b, c). High magnification secondary electron images. $(\mathrm{d}, \mathrm{e}, \mathrm{f})$ SEM-BSE images of tantalum rich inclusion in the etched sample surface. 


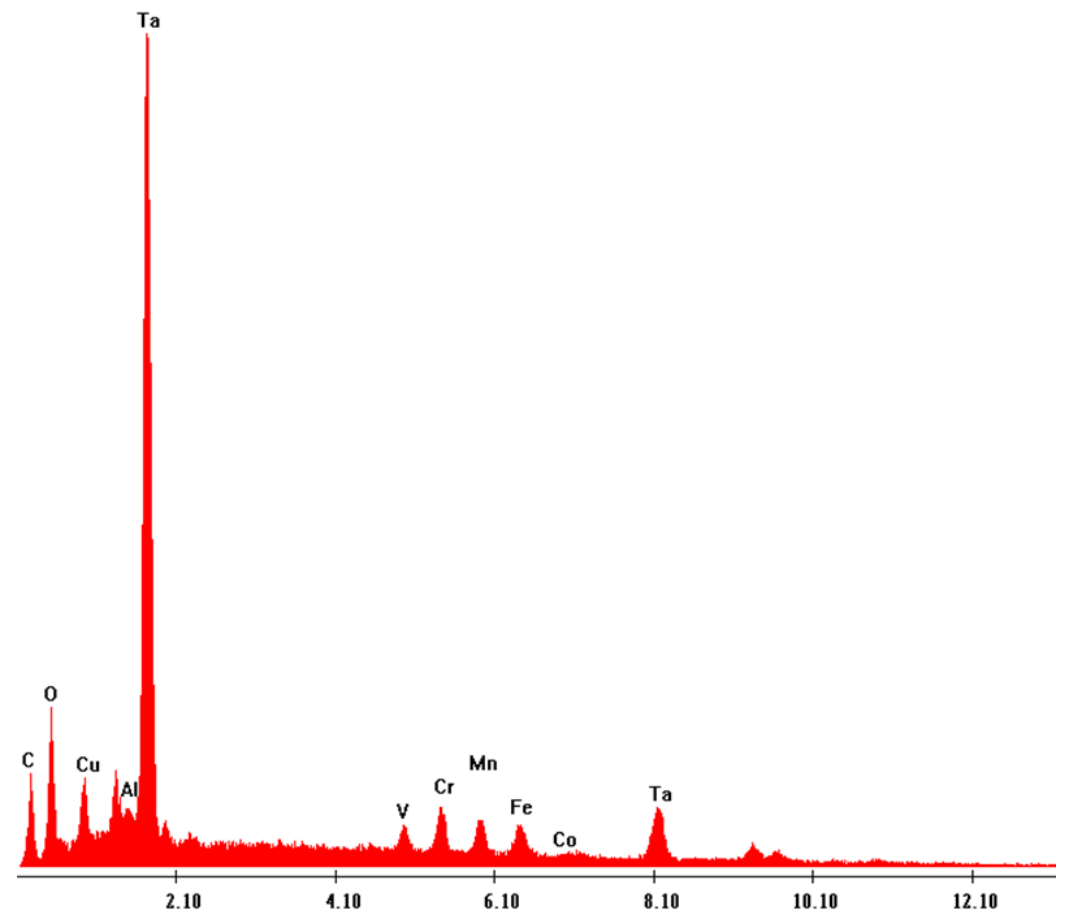

Fig. 30. EDX spot spectra in a SEM from a Ta rich inclusion detected in Fig. 29e: I series steel. X-axis is Energy (keV) and y-axis presents X ray counts. EDAX Genesis EDX system coupled to Hitachi S4800

SEM was used.

TEM/STEM characterization and EDX mapping was performed to image the microstructural features at nano-scale. This step was especially aimed at mapping the carbide/nitride/carbo-nitrides expected in the RAFM steels such as $\mathrm{M}_{23} \mathrm{C}_{6}$ and MX phases. Fig. 31 presents two BF TEM images from $\mathrm{H}$ series steel where some large precipitates $(>100 \mathrm{~nm})$ were detected. Figs. 32-33 present STEM-HAADF images and the associated EDX maps taken from two different FIB foils. In these maps, two types of precipitate families were visible (i) predominantly $\mathrm{Ta}, \mathrm{V}$ and $\mathrm{N}$ rich precipitates which are expected to be the MX precipitates and (ii) predominantly $\mathrm{Cr}, \mathrm{W}$ rich phases, which are expected to be $\mathrm{M}_{23} \mathrm{C}_{6}$ carbides. Because all the strong carbide formers typically enter $\mathrm{M}_{23} \mathrm{C}_{6}$ phase, these particles also showed presence of $\mathrm{Ta}$ and $\mathrm{V}$. It should be noted that mapping low $\mathrm{Z}$ elements like $\mathrm{C}$ and $\mathrm{N}$ are not accurate using EDX, even with Chemi-STEM systems. Thus, $\mathrm{C}$ and $\mathrm{N}$ elemental maps only represent qualitative indication and not absolute values.
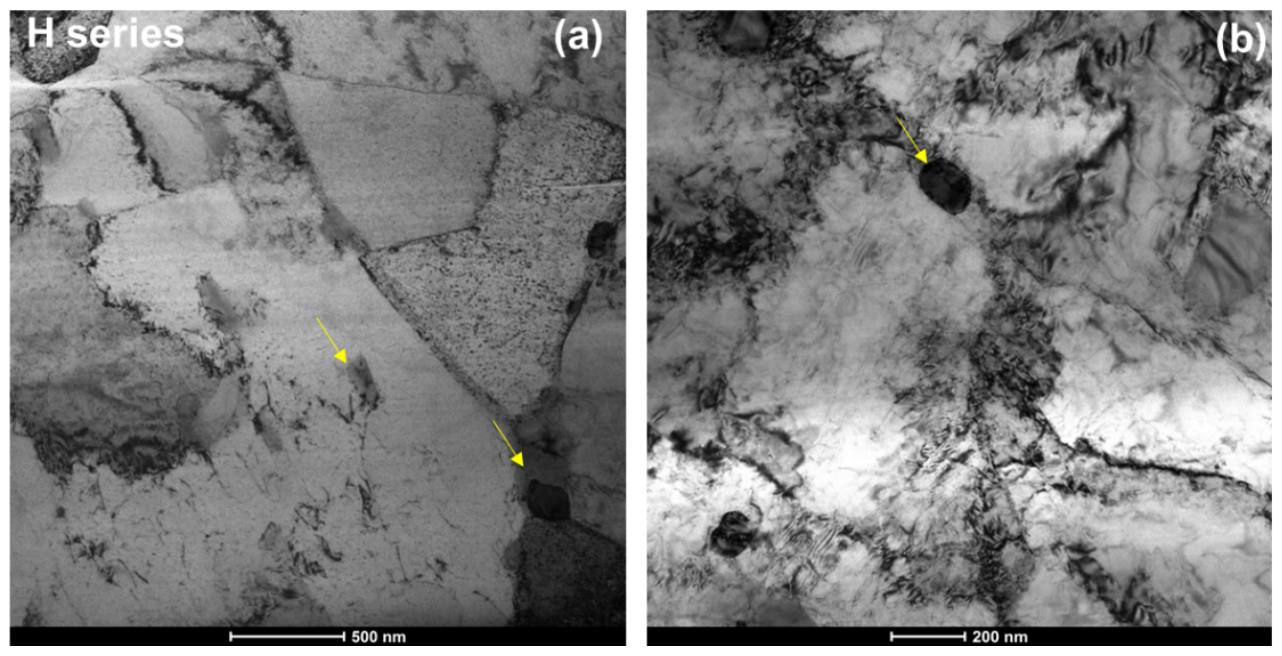

Fig. 31. BF TEM images of $\mathrm{H}$ series steel showing areas from two different samples. 

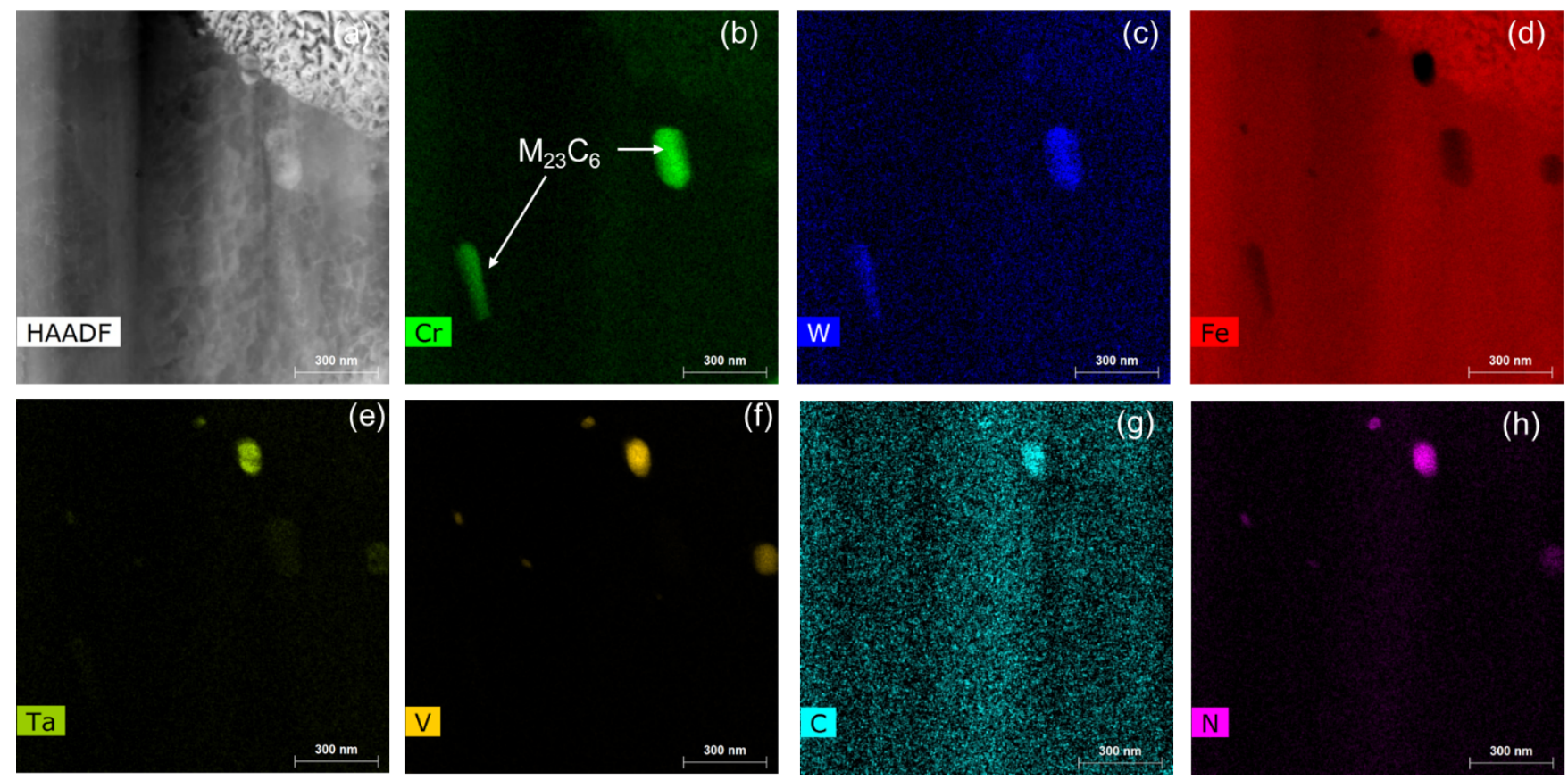

Fig. 32. EDX mapping in STEM mode for H series. (a) HAADF image. (b-h) Elemental maps.
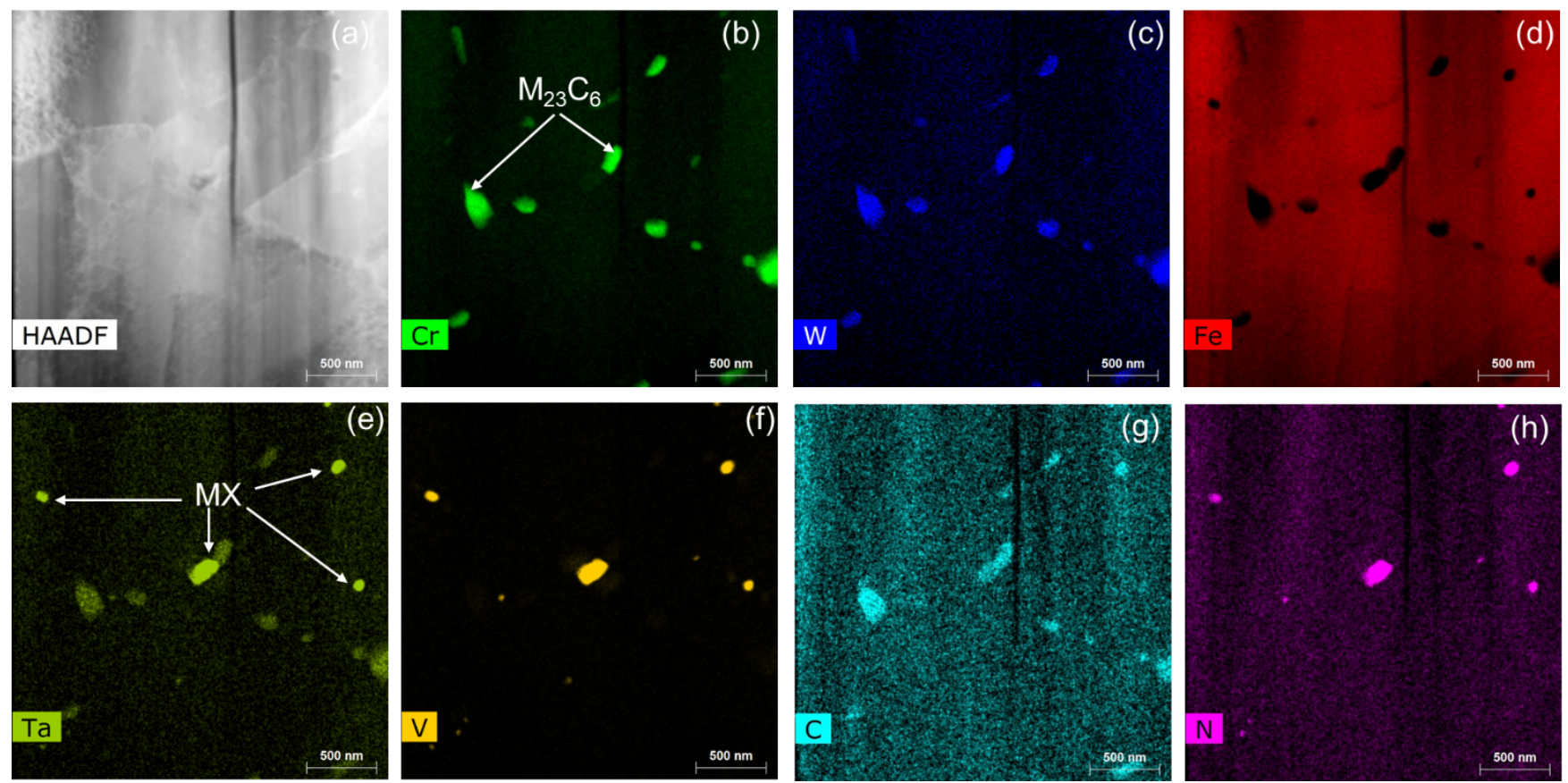

Fig. 33. EDX mapping in STEM mode for H series. (a) HAADF image. (b-h) Elemental maps, second FIB foil 

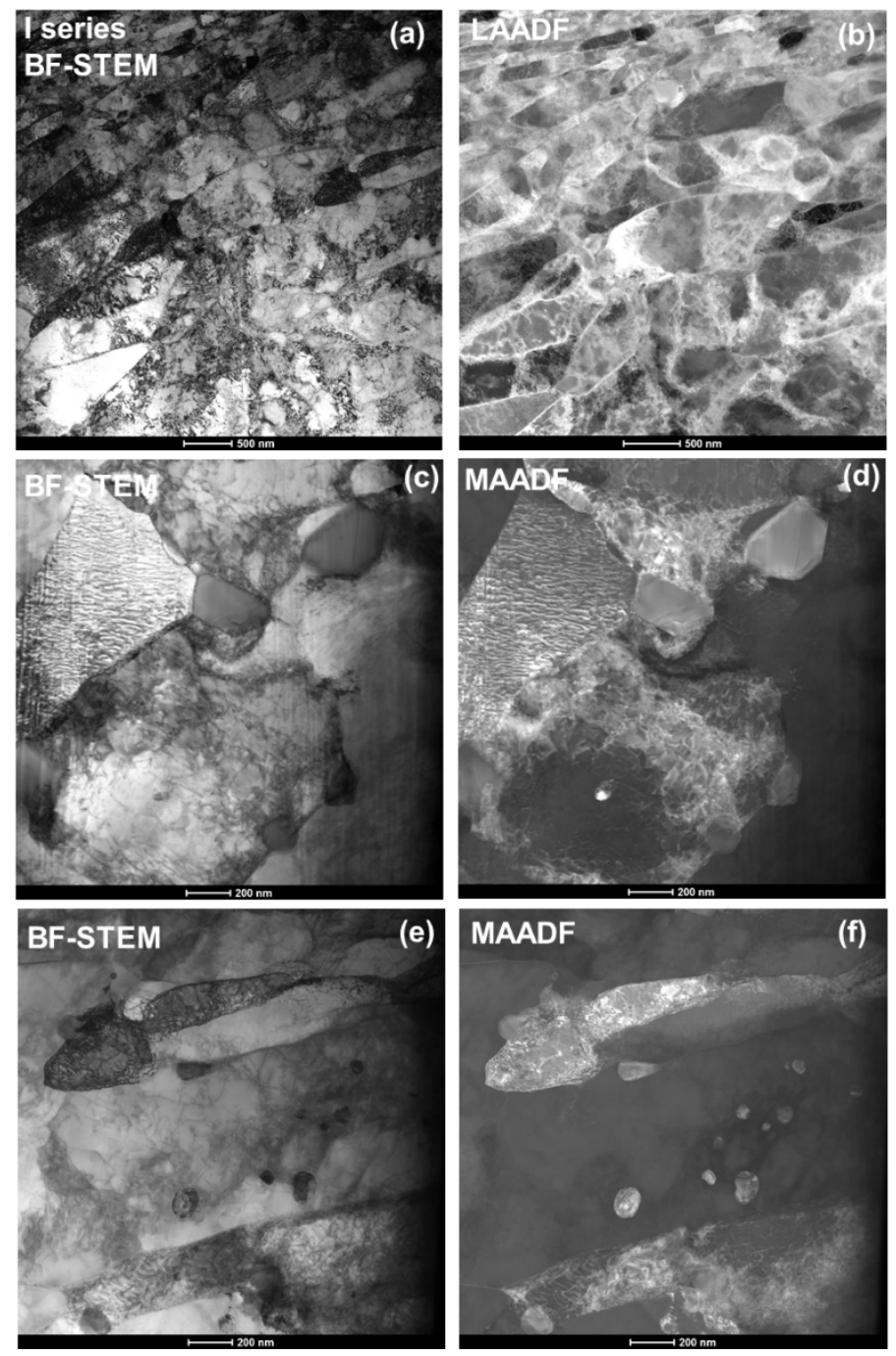

Fig. 34. Overview of I series steel. (a, b) STEM-BF and low-angle annular dark field (LAADF) images showing the latch structure. (c, d) STEM-BF and medium-angle annular (MAADF) image where two large precipitates can be seen in contrast.

For I series steels, a general overview of the microstructure is show in Fig.34. Figs. 34a-34b present STEM-BF and low angle annular dark field (LAADF) image of the same zone revealing the lath/grain structure. Because of the complexity of the microstructure mainly owing to high dislocation density expected in F-M steels, precipitates were not clearly visible in these two images. Figs. 34c-34f present STEM-BF and medium angle annular dark field (MAADF) images where some large precipitates can be seen in contrast. Figs 35 and 36 present EDX maps in STEM mode taken from two different foils of I series steels. In these images the $\mathrm{M}_{23} \mathrm{C}_{6}$ and $\mathrm{MX}$ precipitates were easily distinguishable based on the elemental enrichment: $\mathrm{MX}$ are expected to be enriched in Ta, $\mathrm{V}, \mathrm{N}$ while large $\mathrm{M}_{23} \mathrm{C}_{6}$ carbides contained typically all the carbide formers, but mainly $\mathrm{Cr}, \mathrm{W}$. 

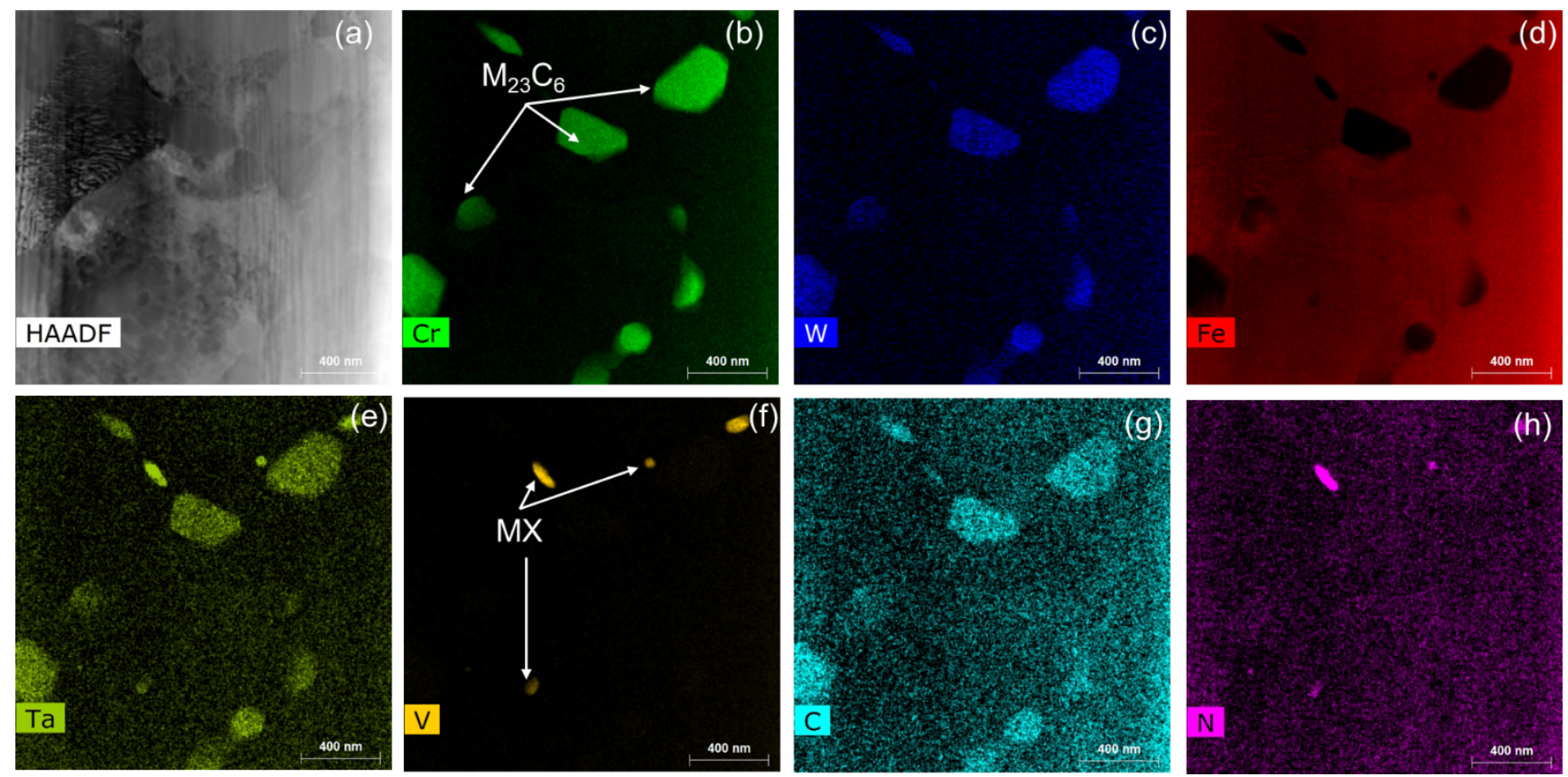

Fig. 35. EDX mapping in STEM mode for I series. (a) HAADF image. (b-h) Elemental maps.
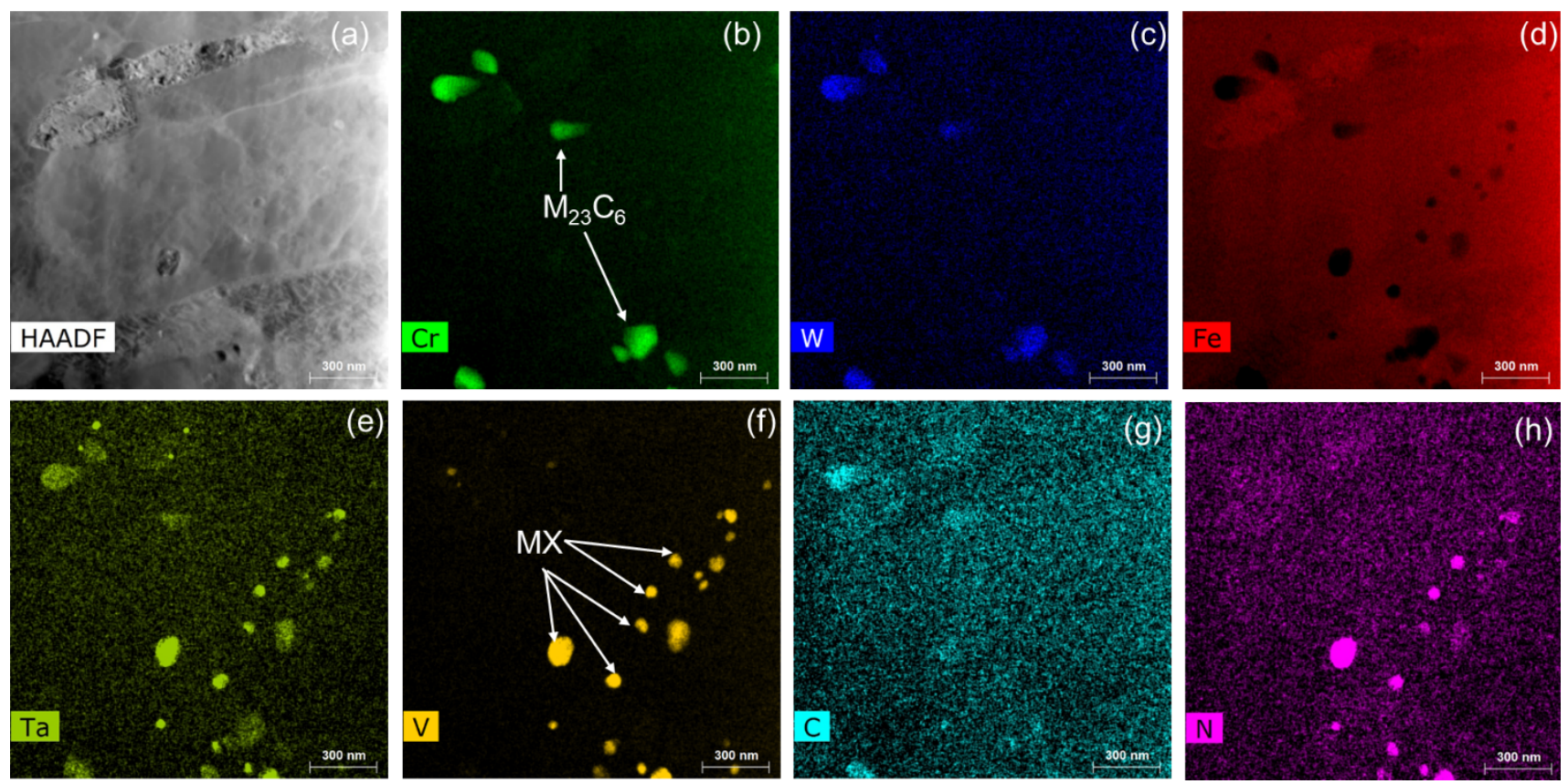

Fig. 36. EDX mapping in STEM mode for I series. (a) HAADF image. (b-h) Elemental maps, second FIB foil.

For P series steel, some TEM and STEM images of the overall microstructure are presented in Fig. 37. Fig. 37a shows an overview in BF-TEM imaging mode. Figs. 37b-37c show the dislocation density within the laths in the P series steel (BF-STEM images). Some large precipitates identified in these images are indicated using arrows. Figs. 37d-37f show BF-STEM, MAADF and HAADF images of the same zone at relatively low magnifications. HAADF images identified some precipitates appearing bright on a dark background (encircled), which are expected to the Ta, V rich MX precipitates. EDX mapping of the precipitates on the same zone on which STEM imaging was performed are shown in Fig. 38. These maps clearly reveal that the bright precipitates detected in HAADF in Fig. $37 \mathrm{f}$ were indeed Ta, $\mathrm{V}$ rich precipitates 
and hence are the MX phase. $\mathrm{Cr}$, W rich large $\mathrm{M}_{23} \mathrm{C}_{6}$ carbides are also indicated in Fig. 38, which were not easily recognizable in the BF, MAADF and HAADF images in Figs. 37d-37f.
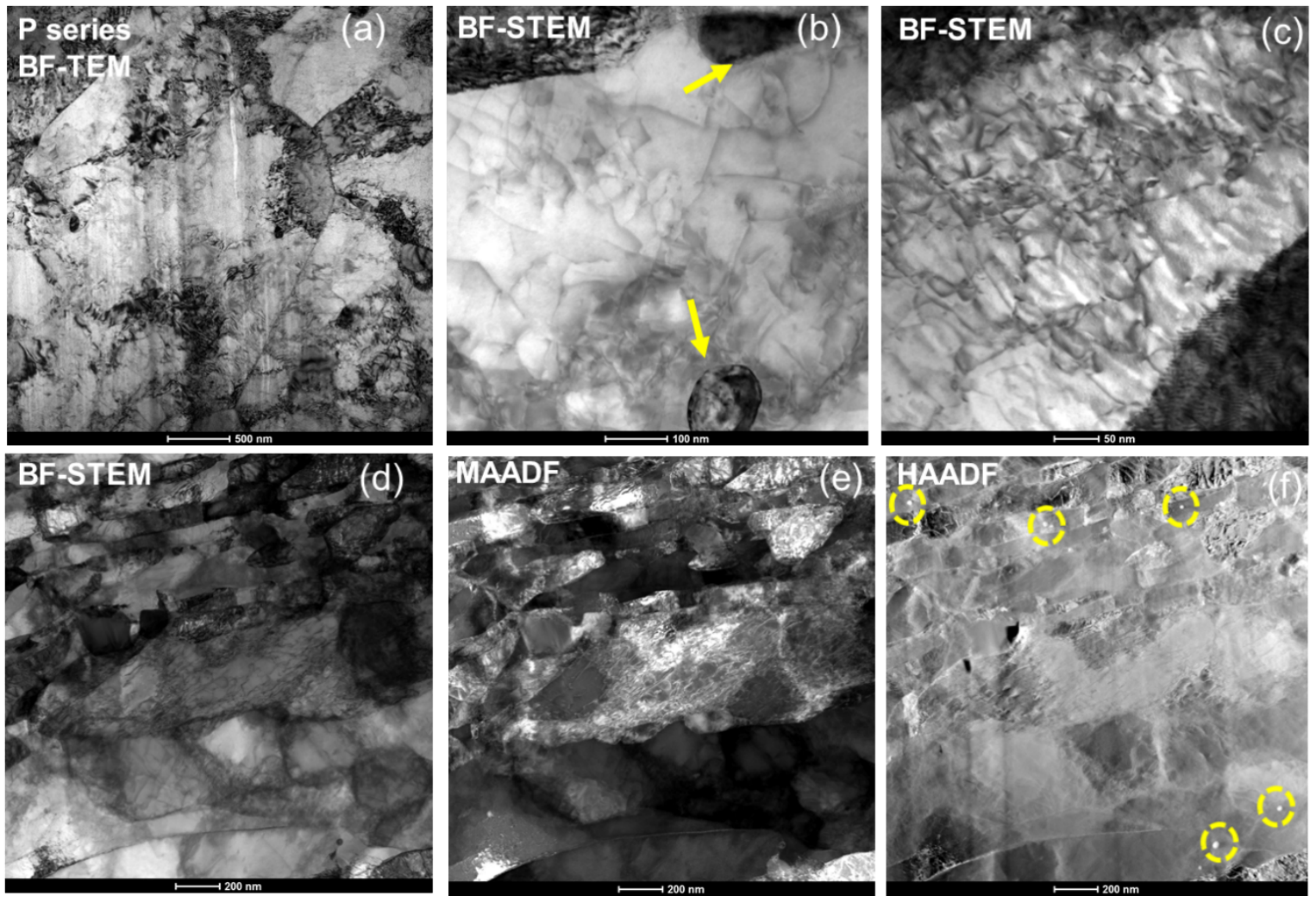

Fig. 37. Overview of P series steel. (a) BF-TEM image. (b,c) BF-STEM images showing the high dislocation density and precipitates. (d, e, f) STEM-BF, MAADF and HAADF images of the same zone. Some bright precipitates in HAADF are encircled.
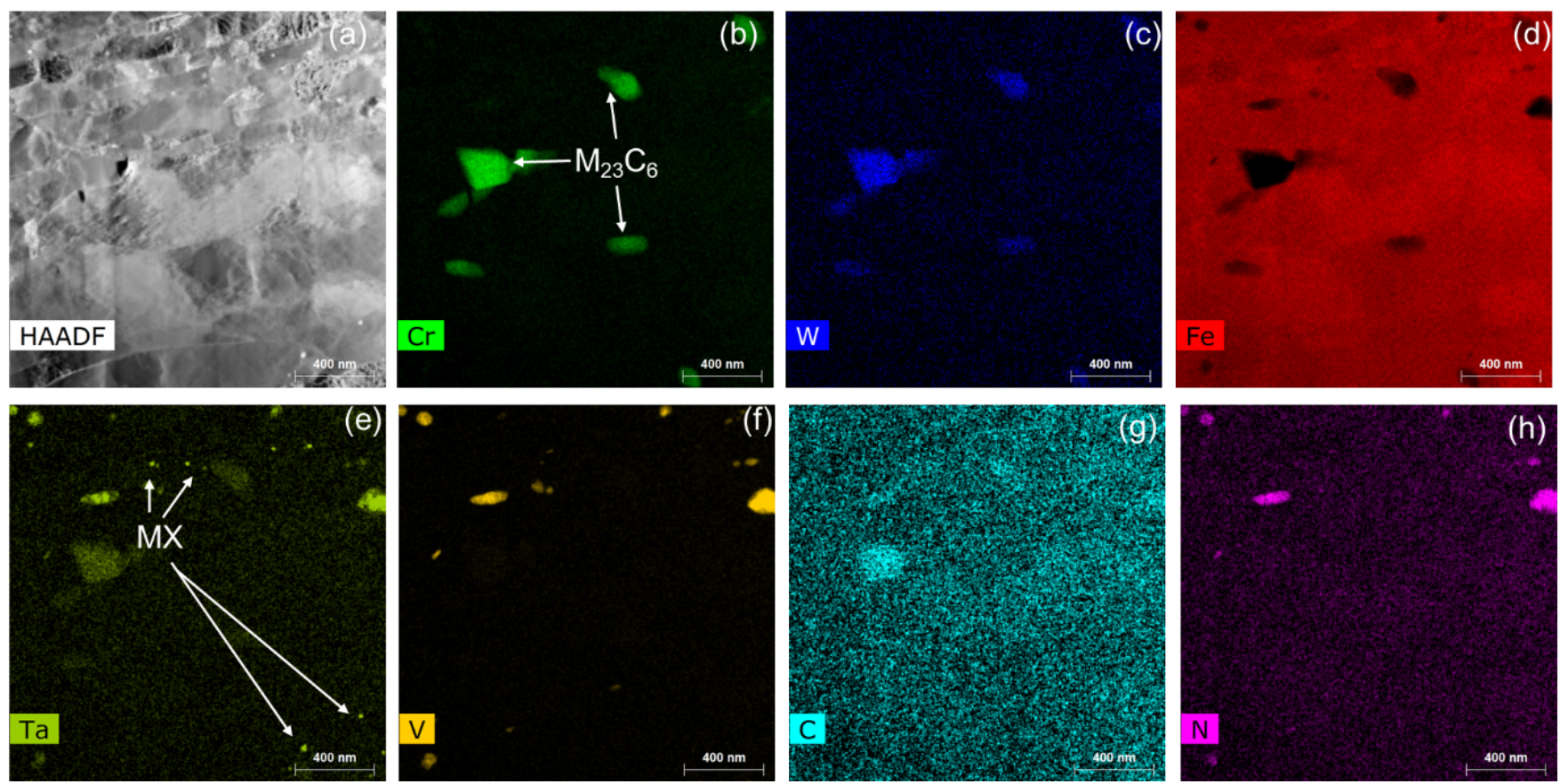

Fig. 38. EDX mapping in STEM mode for P series. (a) HAADF image. (b-h) Elemental maps. 


\subsubsection{J and K series steels: SCK.CEN}

$\mathrm{J}$ and $\mathrm{K}$ series steels microstructures were noted to very different to each other, mainly due to the difference of heat treatment information provided by KIT. J series steels consisted of rather fine grain microstructure. Fig. 39 presents an LOM image of the steel (after etching using Villela's reagent). Black dots in the image are expected to be the carbides in the steel forming as a result of the tempering treatment. PAGs were not easily recognizable in this steel in the LOM images.

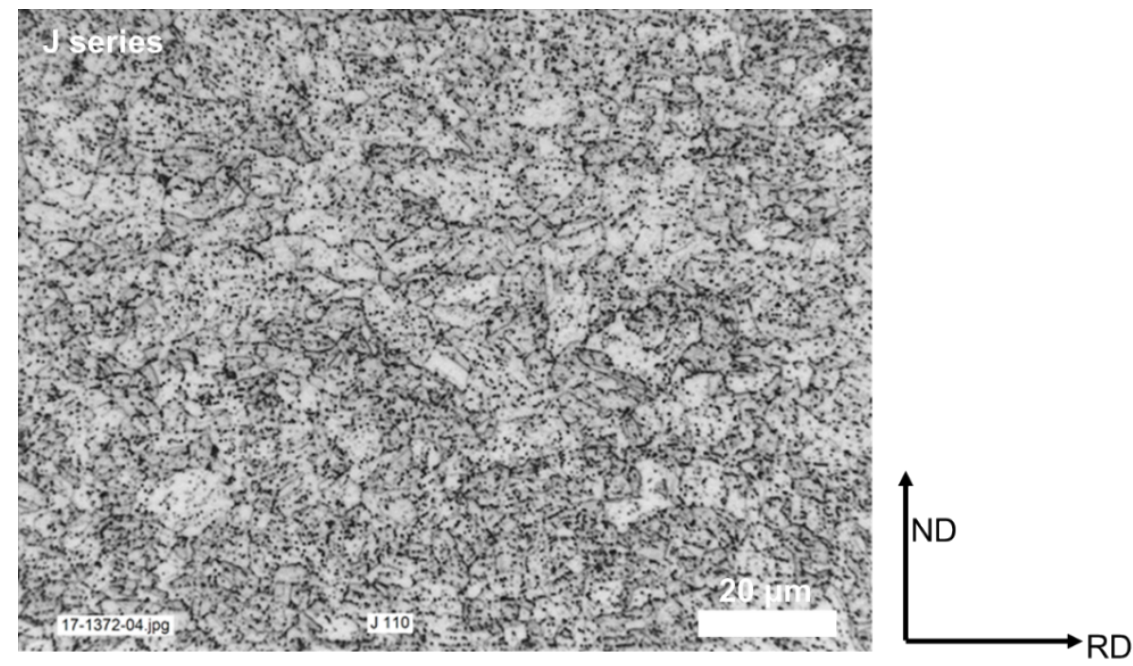

Fig. 39. LOM of J series steel after etching using Villela's reagent.
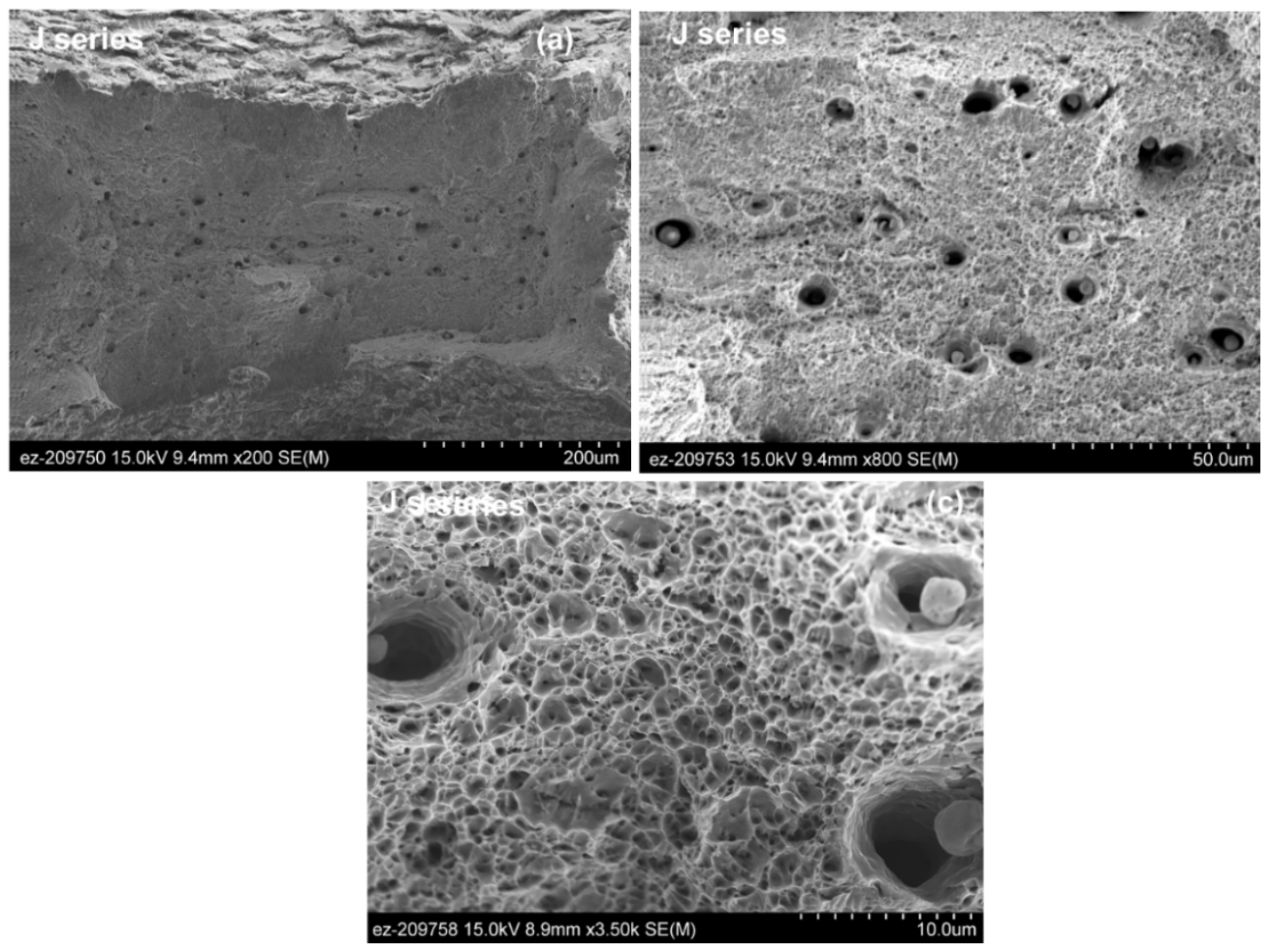

Fig. 40. Fractography of $J$ series steel after tensile tests (secondary electron images). (a) Fracture surface overview. (b) Inclusions on the fracture surface and (c) Ductile fracture surface with cup and cone features. 
After tensile deformation, $\mathrm{J}$ series steel failed in a ductile fashion and consisted of cup and cone features. However, many large inclusions were identified on the fracture surface, which caused much deeper areas of cup and cone type fracture. Fig. 40a presents the fracture surface overview. Fig. 40b presents a relatively higher magnification image showing the inclusions inducing cup and cone fracture. Some inclusions can be seen embedded in the burrows. Fig. 40c shows the typical dimpled fracture surface expected from ductile materials. SEM BSE images on polished-etched surface identified inclusion in the steel which appeared similar to those detected in H, I and P series steels (Fig. 41). Because these inclusions appeared bright in BSE images, they are also expected to be tantalum rich oxides which are not uncommon in RAFM steels. These inclusions are expected to be the same type as those detected on the fracture surfaces.

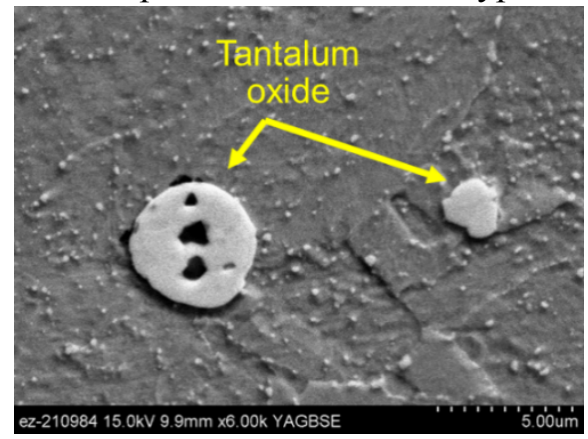

Fig. 41. Inclusions in J series steels. SEM-BSE image on etched surface. Dark areas are holes on the inclusion surface, presumably due to acid attack.

Fig. 42 presents BF TEM/STEM and MAADF images of the microstructure of J series. In all these figures the laths can be identified with typical large precipitates. Precipitates were better resolved in the MAADF images (indicated using yellow arrows). To better distinguish the $\mathrm{M}_{23} \mathrm{C}_{6}$ and $\mathrm{MX}$ phases, EDX mapping was performed in STEM mode (see Figs.43-44). Large precipitates ( $>50-80 \mathrm{~nm}$ ) were $\mathrm{Cr}, \mathrm{W}$ rich and are expected to be $\mathrm{M}_{23} \mathrm{C}_{6}$, while smaller precipitates $(<30 \mathrm{~nm})$ where Ta, $\mathrm{V}$ and $\mathrm{N}$ rich consistent with MX phases. Figs. 43-44 also further highlight that in F-M steels, all the precipitates are seldom visible using only TEM/STEM imaging. Therefore, it is important to perform EDX mapping.
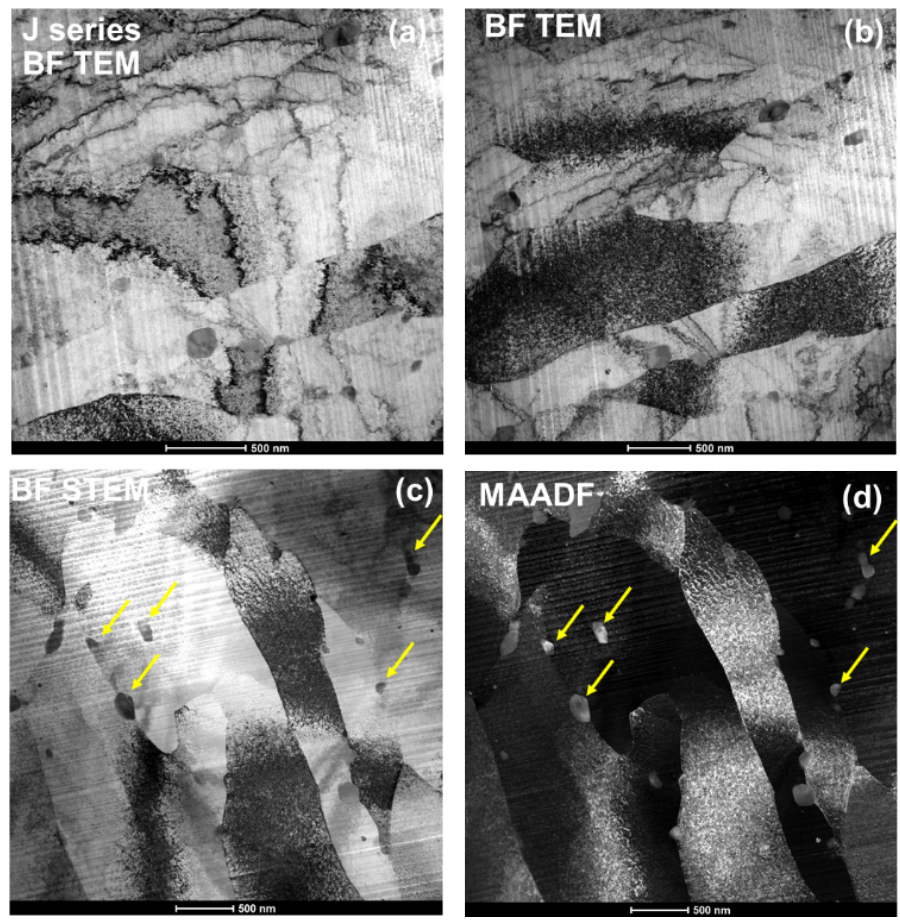

Fig. 42. TEM and STEM imaging of J series (a,b) Conventional BF TEM images, (c, d) BF STEM and MAADF image of the same zone. 

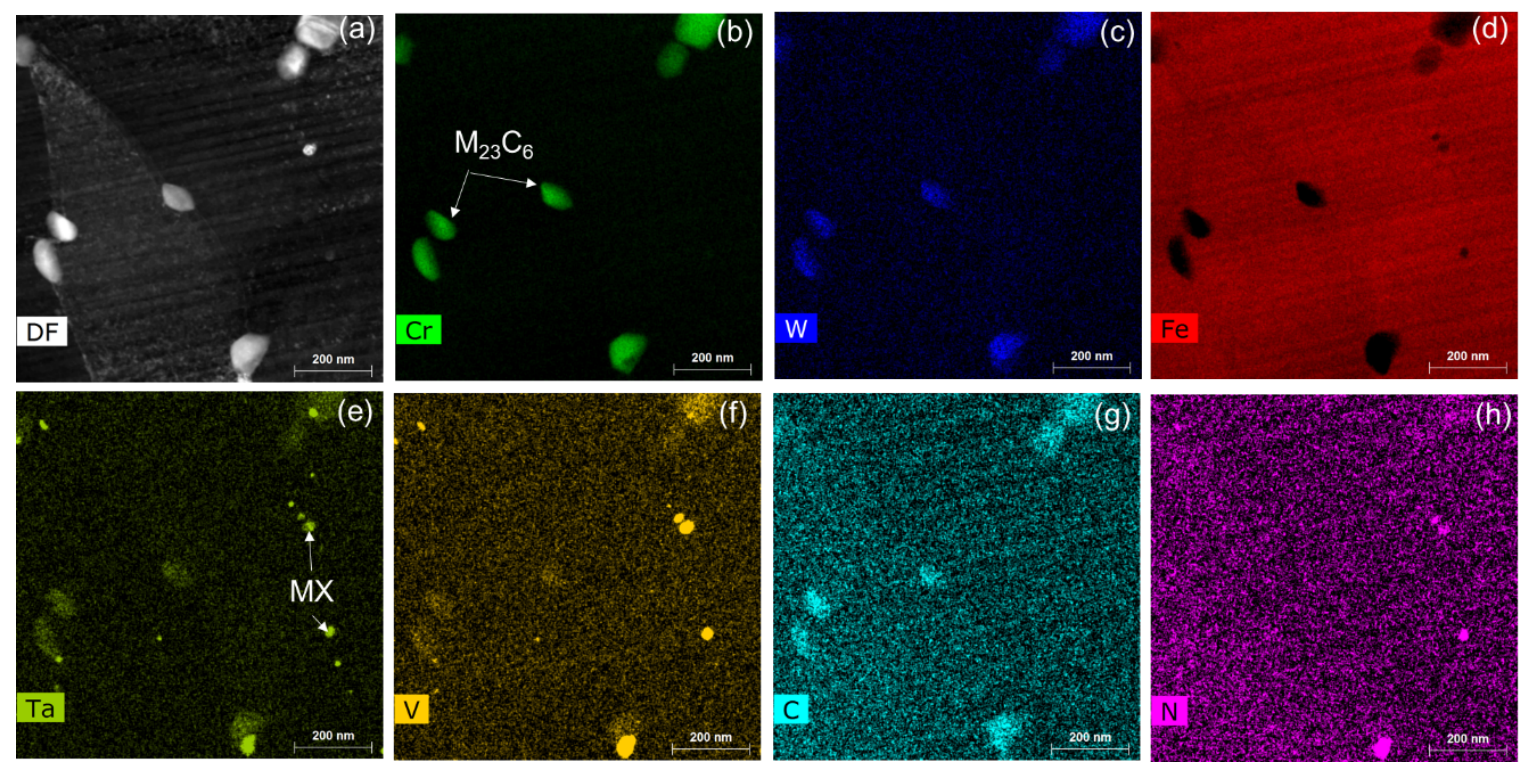

Fig. 43. EDX mapping in STEM mode for J series. (a) MAADF image. (b-h) Elemental maps.
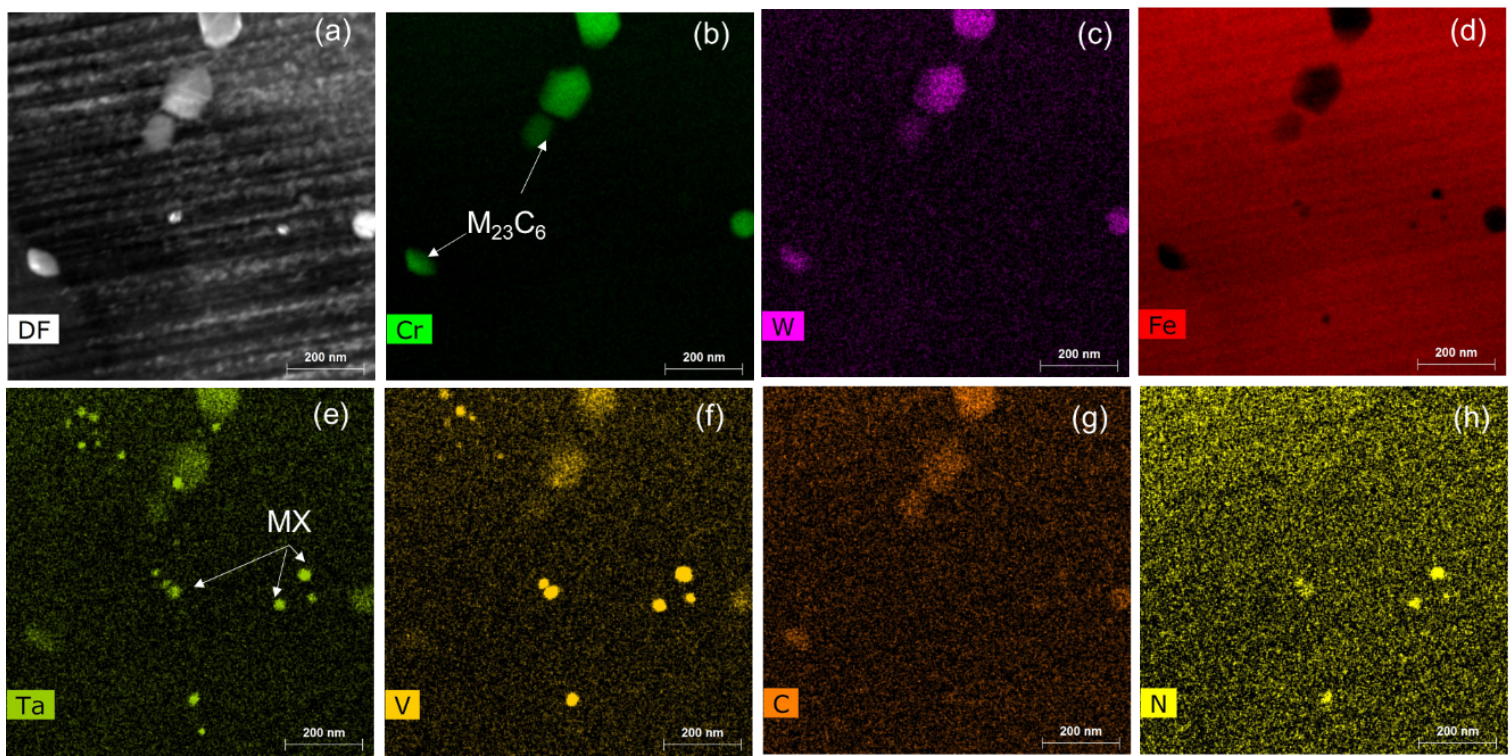

Fig. 44. EDX mapping in STEM mode for J series: different FIB foil. (a) MAADF image. (d-h) Elemental maps.

$\mathrm{K}$ series steel consisted of very large PAGs, which can be attributed to the high normalizing temperature of $1050{ }^{\circ} \mathrm{C}$. This can be seen in the LOM images in Fig. 45. Grain size appeared to be bimodal, But the overall microstructure resembled an under-tempered steel. Typically, untempered/under-tempered martensitic regions appear much brighter than tempered areas after etching, because the carbides have not yet formed in these regions. Fig. 45b is indicative that the entire PAGs were not fully tempered to reveal well-defined lath structures, as was seen for L series in Fig. 19. This is expected because the tempering temperature provided by KIT is $675^{\circ} \mathrm{C}$ for this steel: very low compared to $760{ }^{\circ} \mathrm{C}$ for F-M steels. Fig. $45 \mathrm{~b}$ shows a relatively high magnification image of regions where some laths can be identified, while the rest of the area appears untempered or under-tempered - yellow arrows point to regions where some lath structure was visible while red arrows point to areas where no laths were identified and only some etch pits can be seen. We must highlight that this steel was also the most difficult to properly etch to reveal the microstructure. As a result, slight over etching was required to sufficiently reveal the features. 

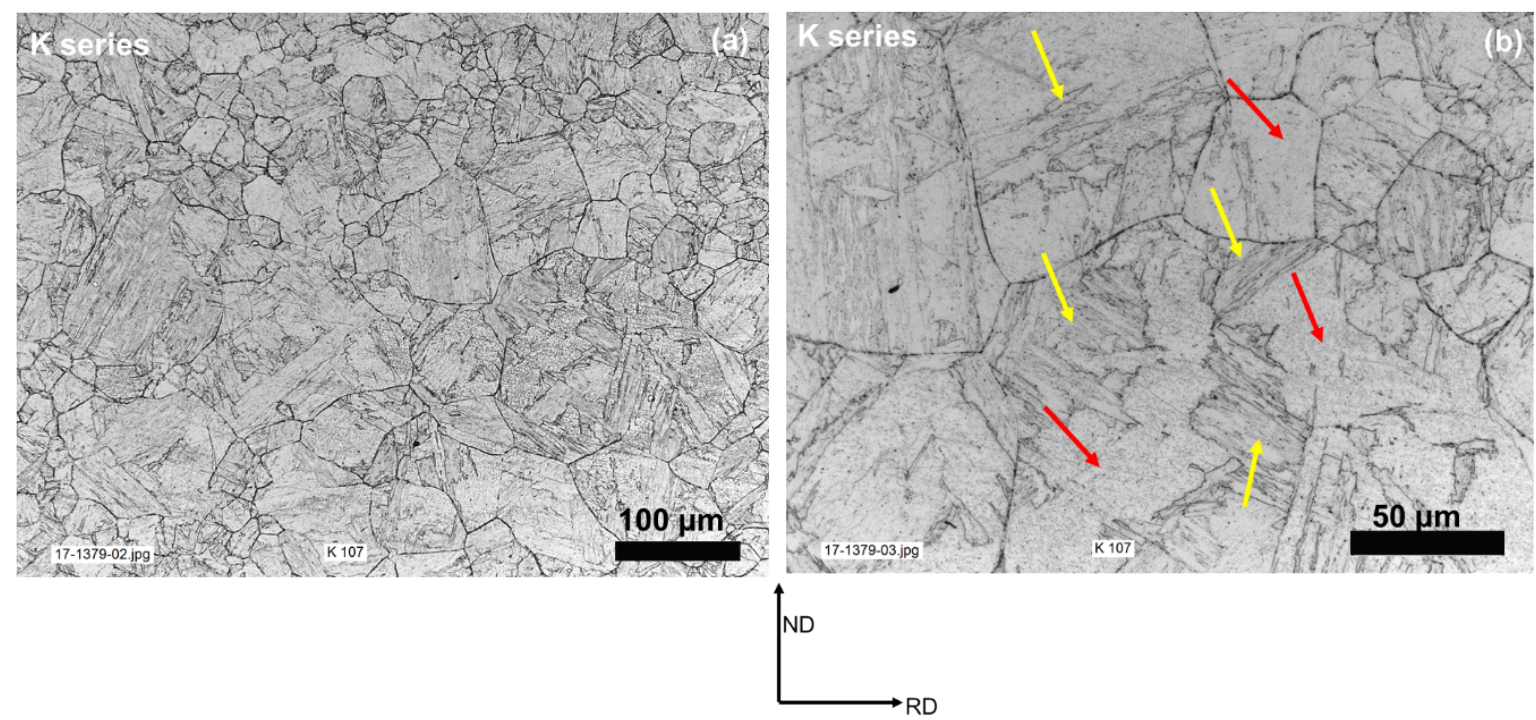

Fig. 45. LOM images of K series steel after etching in Villela's reagent (a) Low magnification image. (b) Image showing the areas where laths are developed (yellow arrows) and regions where no laths were identified optically, representing under tempered regions (red arrows).

A lack of precipitates was also evident in the SEM images where very few matrix precipitates were detected (Fig. 46). Some precipitates, appearing as short "films" on the grain boundaries were observed. These are perhaps the initial stages of the formation of $\mathrm{M}_{23} \mathrm{C}_{6}$ phase on the grain boundaries. Some identified grain boundary and matrix precipitates are encircled in Fig. 45.
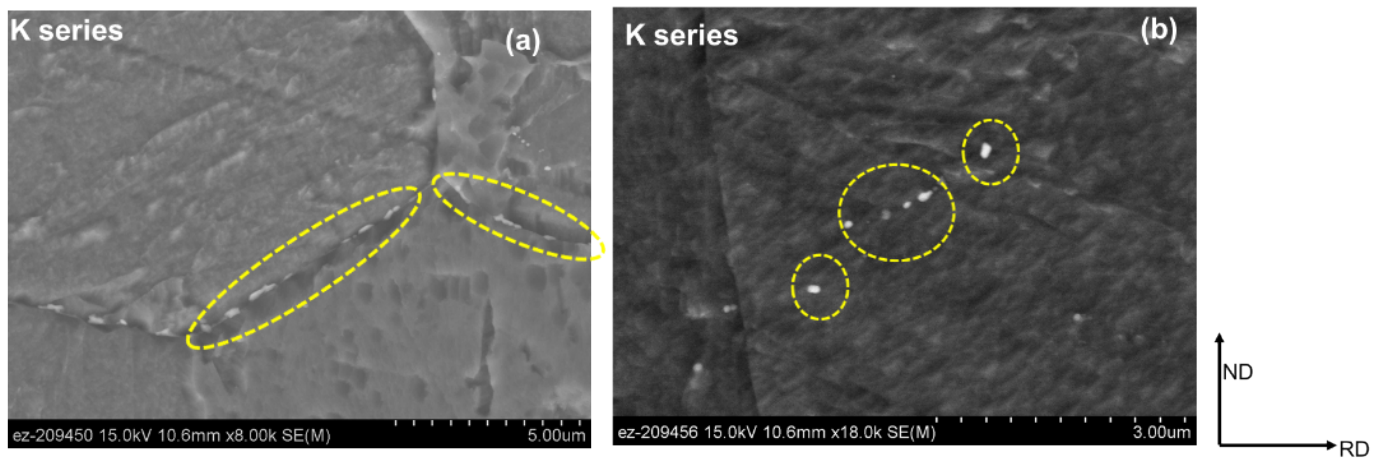

Fig. 46. SEM secondary electron images showing some identified precipitates in K series steel. (a)

Precipitate films on grain boundaries and (b) Some precipitates in matrix.

To further confirm a lack of precipitates in the $\mathrm{K}$ series steel TEM/STEM imaging and EDX mapping was performed. Fig. 47 presents two BF TEM images and a MAADF image of different areas. In these figures, so obvious precipitates were detected, as was seen previously for the other steels: for example Fig. 42 for J series. Small black dots in Fig. 47 are expected to be FIB milling induced defects. As we have seen previously, only TEM/STEM imaging may not be sufficient to reveal precipitates in F-M steels. Thus, STEM-EDX mapping was also performed on the same zone from where the MAADF image was taken in Fig. 47c These maps are presented in Fig. 48, showing the distribution of typical elements in the steel. Once again, no signs of any precipitates was detected. The lath boundaries appeared enriched in $\mathrm{Cr}, \mathrm{V}$ and $\mathrm{Ta}$. Some areas showing a strong $\mathrm{Cr}$ signal are indicated using arrows. EDX mapping was also conducted at higher magnifications to see if low magnification scans are missing something or not. These maps are presented in Fig. 49. Once again, no obvious sign of precipitates was detected, except some local Cr, V, Ta enrichment, presumably on a lath boundary. This analysis proves that the steel is indeed under-tempered. 
Therefore, either the precipitates have not formed at all or are below the resolution limit of STEM. Perhaps atom probe tomography (APT) will be needed to further confirm the presence of precipitates.

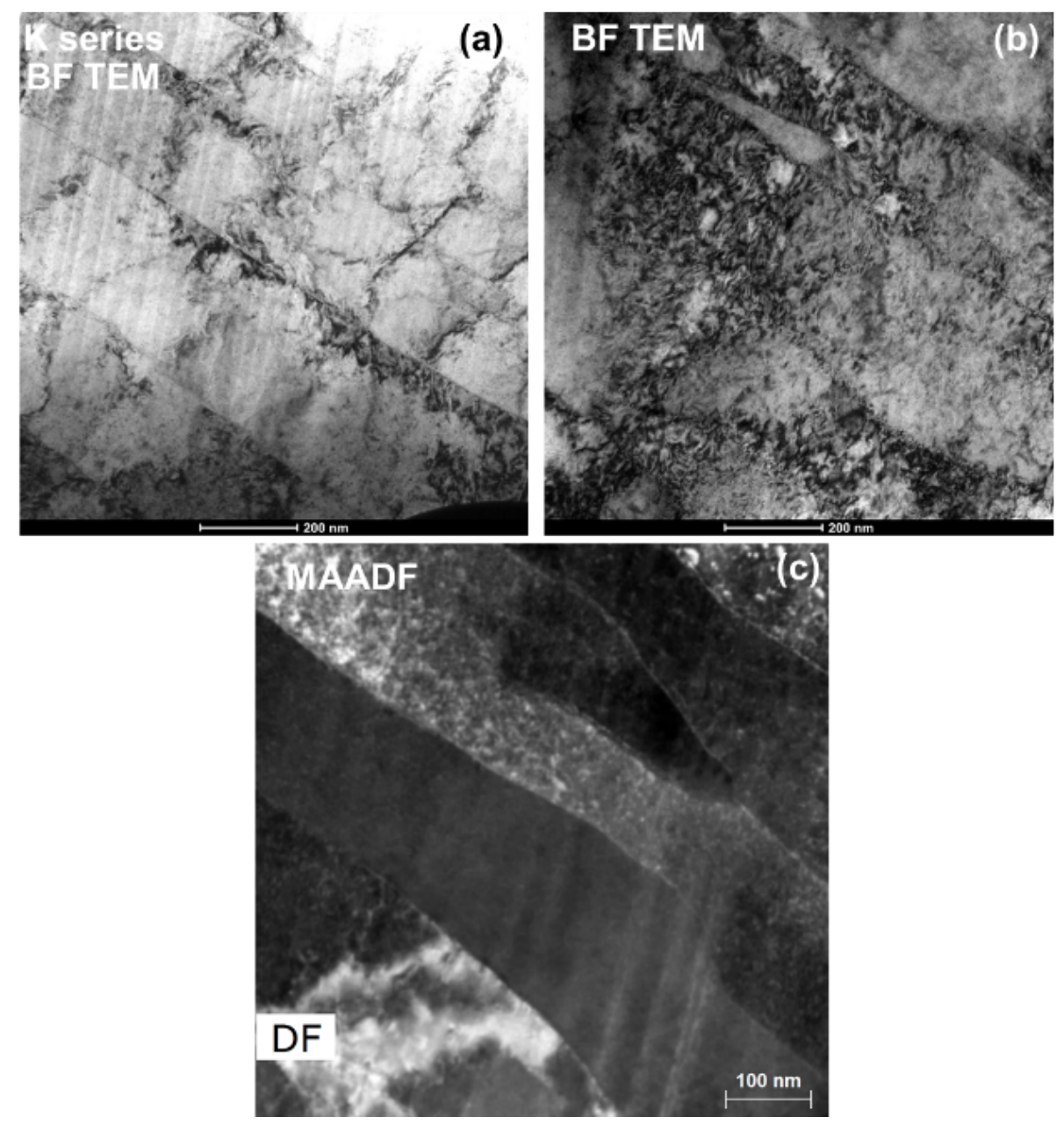

Fig. 47. TEM/STEM imaging of K series steel. ( $a, b)$ BF TEM images (c) STEM-MAADF image. 

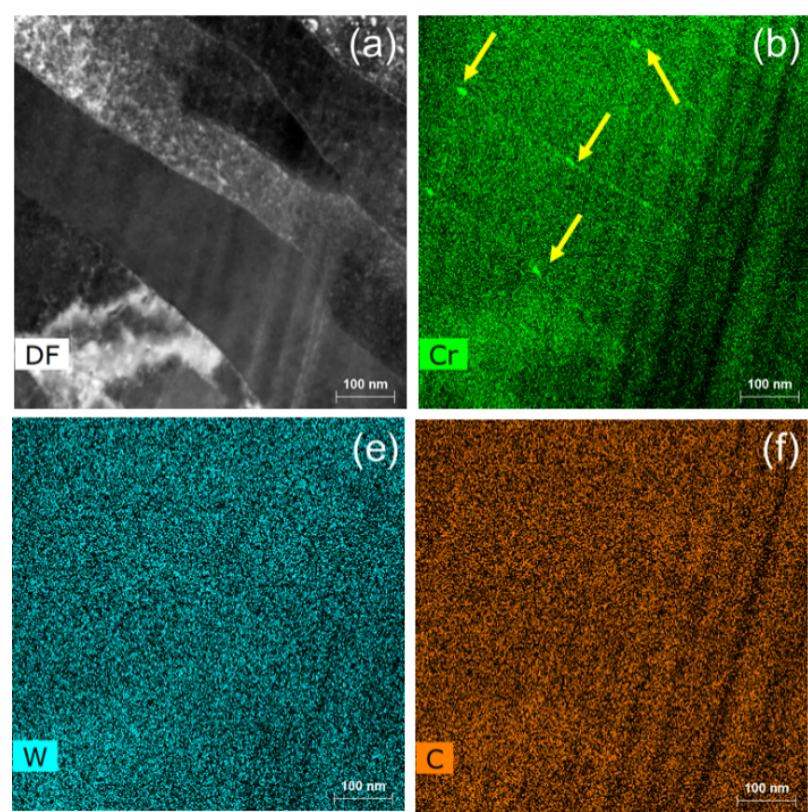
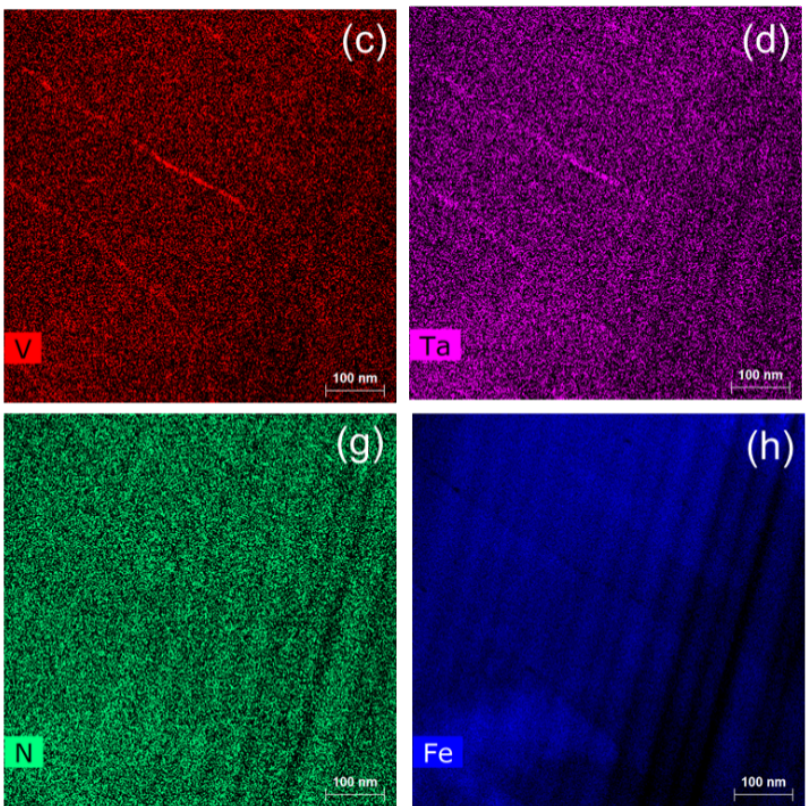

Fig. 48. STEM-EDX mapping of K series steel. (a) MAADF image, (b-h) EDX maps of different elements in the steel. Dark lines at the left of EDX maps are artefacts due to FIB milling curtains. Yellow arrows show some $\mathrm{Cr}$ rich areas on the laths.
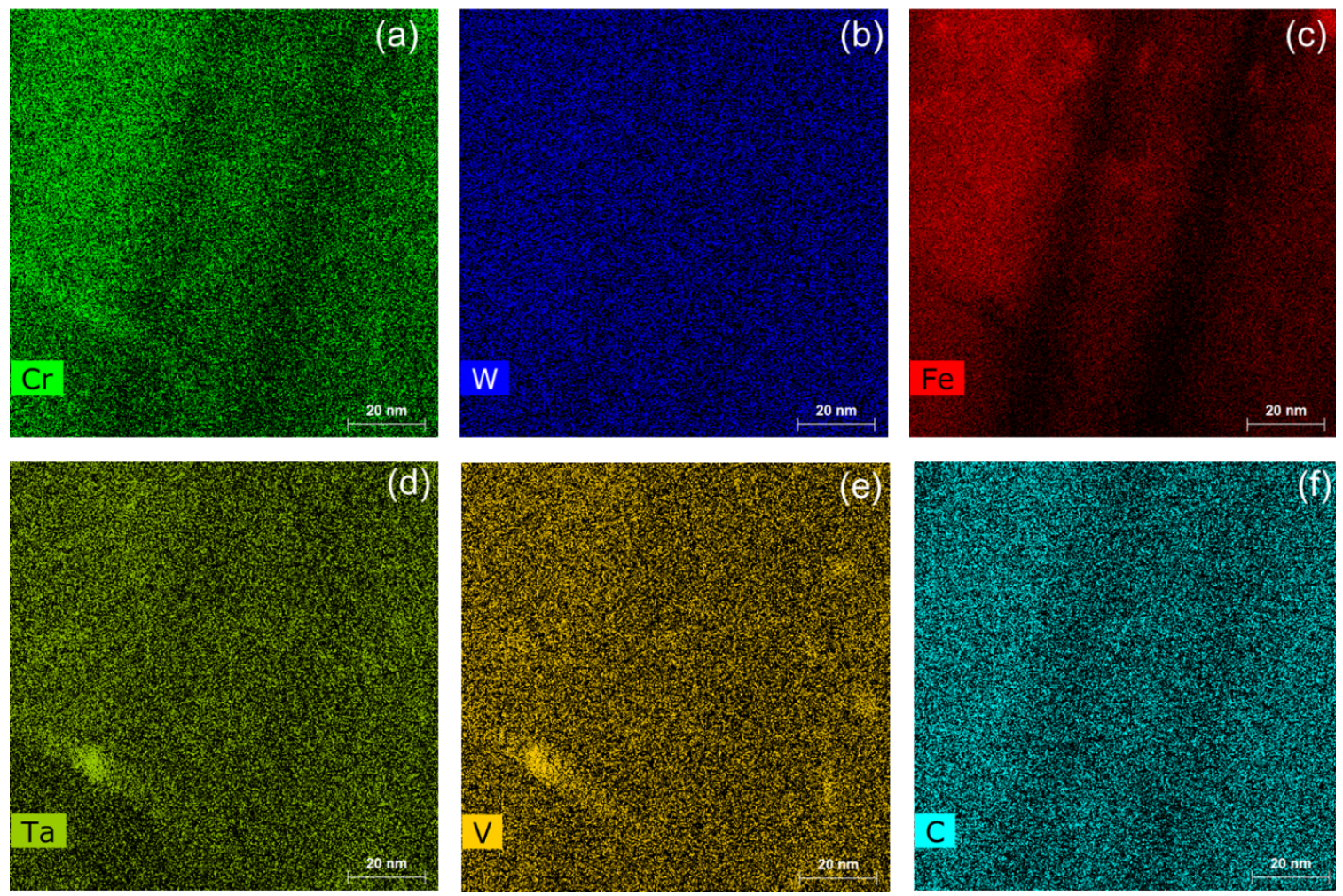

Fig. 49. High magnification STEM-EDX mapping of K series steel, second FIB foil. 


\subsubsection{M, N and O series steels: ENEA}

LOM images of M, $\mathrm{N}$ and $\mathrm{O}$ series steels, after etching in Villela's reagent, are presented in Fig. 50. Between $\mathrm{M}$ and $\mathrm{N}$, which are the double austenised versions, there was no striking visible difference. However, grains in $\mathrm{M}$ series did appear slightly more refined than in $\mathrm{N}$ series. In both these steels, the optical microstructures resembled a typical fully tempered F-M steel with laths and precipitates (appearing as black dots).

$\mathrm{O}$ series steel microstructure was different when compared with $\mathrm{N}$ and $\mathrm{M}$. This steel comprised of grains elongated along the plate rolling direction. The observation is consistent with the rolling deformation information provided about the steel $\left(650^{\circ} \mathrm{C}\right.$ hot rolling- $40 \%$ reduction).

Upon tensile deformation, all the three steels failed in a ductile fashion (Fig. 51). RA of $\mathrm{N}$ series was the highest, as can be inferred from the overview of the fracture surface in Fig. 51b. Fracture surface of $\mathrm{N}$ and $\mathrm{O}$ series steels also showed areas of deep cup and cone features (see Figs. 51e-51f), expected due to the presence of inclusions. In the fracture surface of $\mathrm{N}$ series, two inclusions can be seen inside the burrows. No such inclusions were observed for the M series steel. SEM-BSE imaging identified some inclusions on the etched sample surface of $\mathrm{N}$ and $\mathrm{O}$ steels (Fig. 52), similar to previous steels (H,I,P and J). These inclusions were bright in BSE images, hence must contain heavy elements like Ta and are expected to be oxides as in the previous steels. The inclusions on the fracture surface are expected to be the same type as those detected by SEM-BSE.

TEM/STEM imaging of M series steel are presented in Fig. 53. Figs. 53a-53b show the BF-TEM images of the steel. The precipitates were better visible in the STEM imaging in Figs. 53c-53h where STEM-BF, MAADF and HAADF images are shown. Due to high Ta content, globular MX carbides were easily recognizable in the HAADF images where they appeared bright on a relatively dark background. EDX maps obtained from the same zone on which STEM was performed are presented in Fig. 54, where the bright objects in HAADF images were confirmed to be Ta rich phase, expected to be MX, while the larger precipitates ( $>100-150 \mathrm{~nm}$ ) rich in $\mathrm{Cr}$, W are expected to $\mathrm{M}_{23} \mathrm{C}_{6}$.
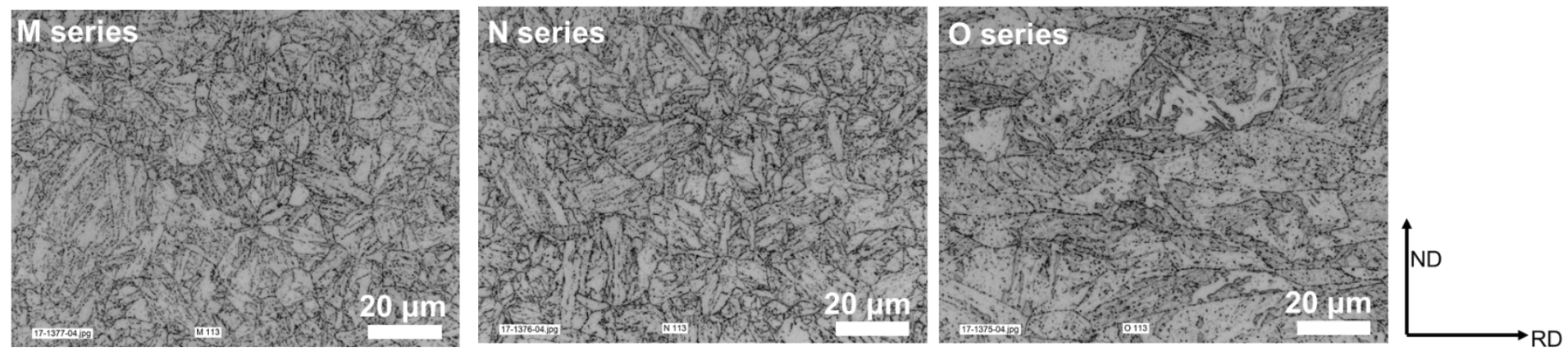

Fig. 50. LOM images of (a) M series (b) $\mathrm{N}$ series and (c) $\mathrm{O}$ series steels. 

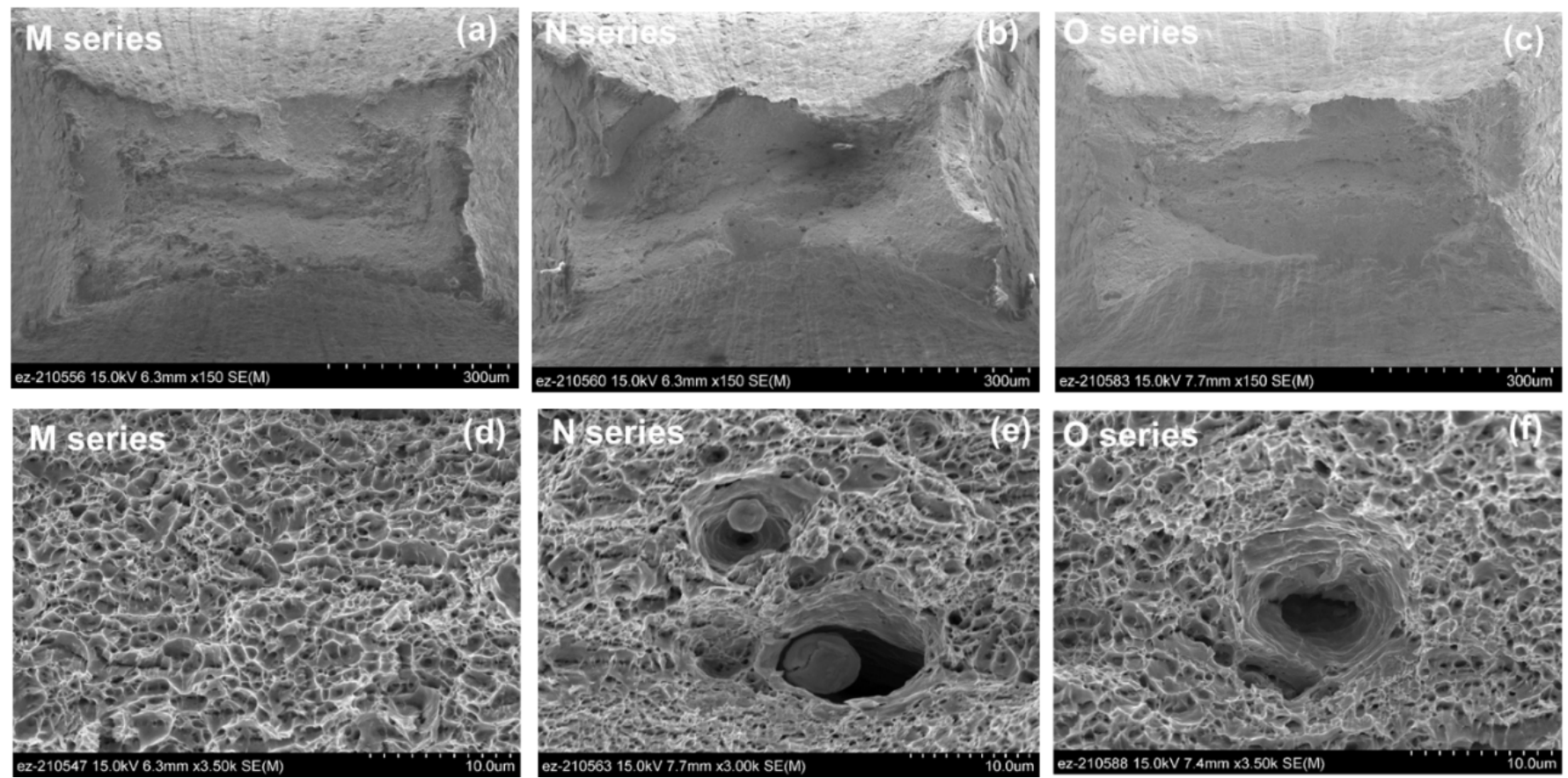

Fig. 51. Fractography on M, N and O series steels using SEM secondary electron imaging. (a-c) Fracture surface overview. (d-f) High magnification images of the fracture surface showing the ductile failure.
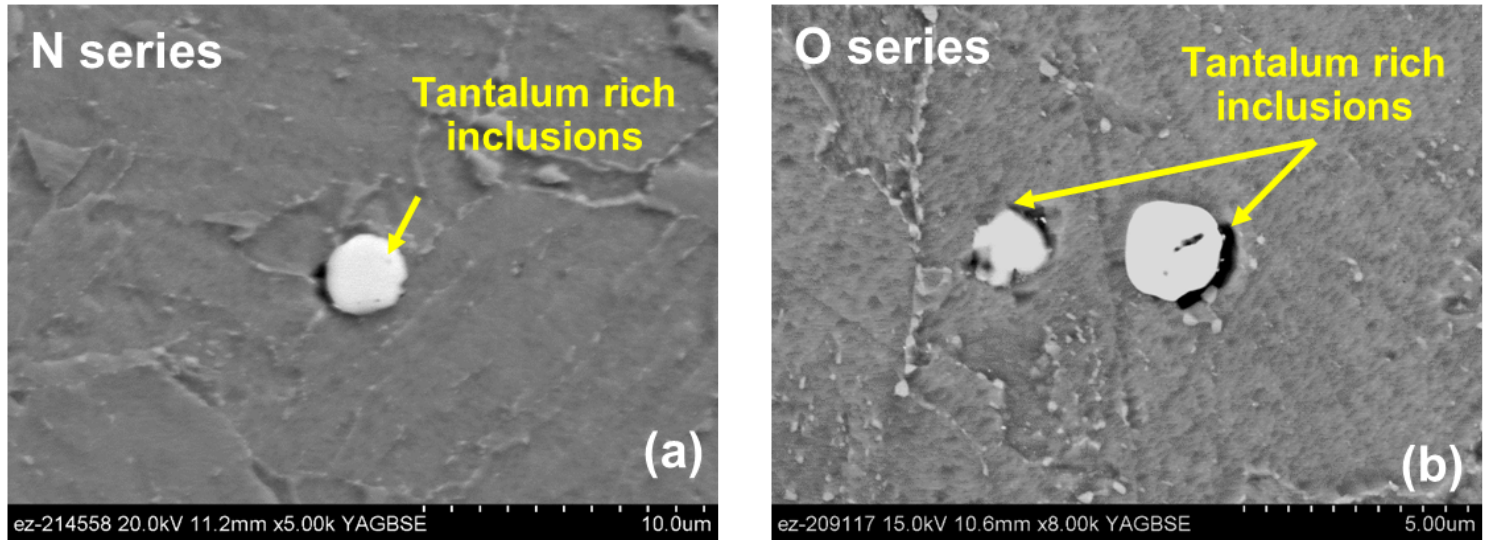

Fig. 52. SEM-BSE images of inclusion in (a) $\mathrm{N}$ series and (b) $\mathrm{O}$ series steels, expected to be Ta rich oxides. 


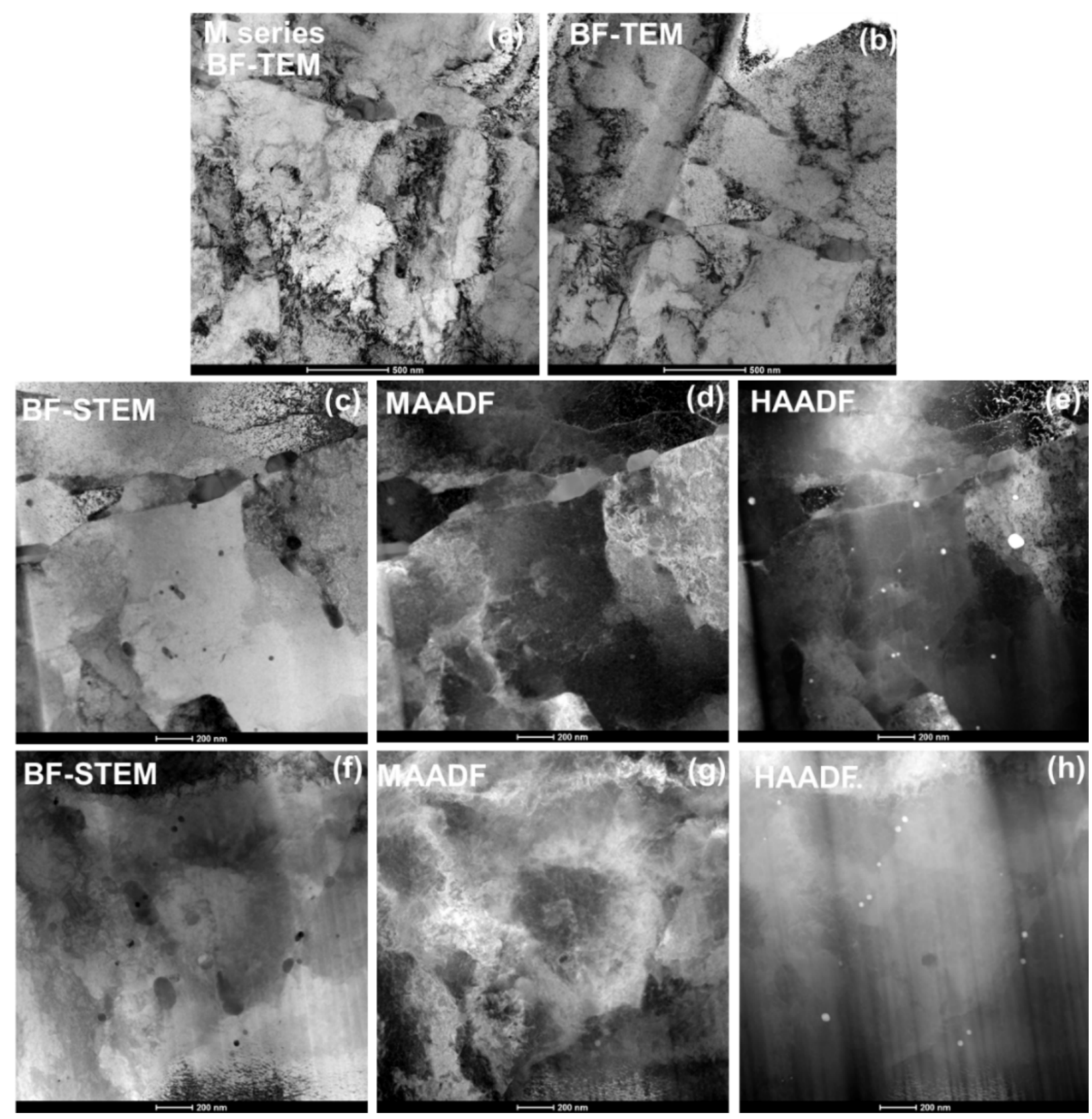

Fig. 53. TEM and STEM imaging of $M$ series steel. ( $a, b)$ BF TEM images. (c, d, e) STEM BF, MAADF and HAADF images of the same zone showing precipitates. (f, g, h) STEM BF, MAADF and HAADF images in a different FIB foil of same zone. 

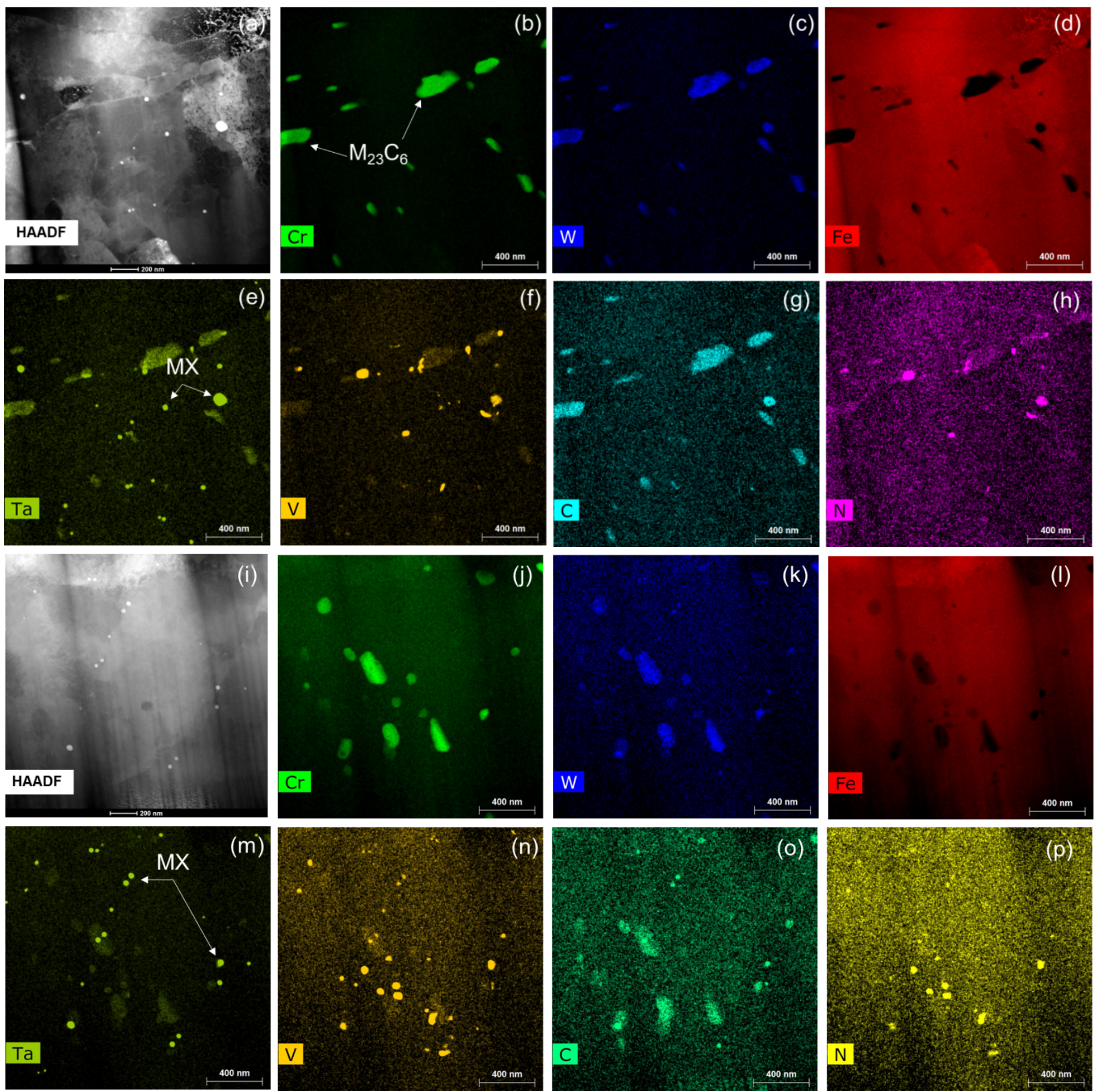

Fig. 54. STEM-EDX mapping of M series steel (a-h) HAADF image and EDX maps of same zone. (i-p) HAADF image and EDX mapping from a different FIB foil.

TEM and STEM analysis of $\mathrm{N}$ series steel is presented in Fig. 55. Figs. 55a-55b show BF-TEM images where some precipitates can be seen. Figs. 55c-55f are STEM-BF and HAADF images showing the distribution of $\mathrm{M}_{23} \mathrm{C}_{6}$ and $\mathrm{MX}$ precipitates. MX appeared brighter in the HAADF images due to high Ta content. EDX maps from the same zones are presented in Fig. 56 where the Ta rich MX particles and $\mathrm{Cr}$, 
W rich $\mathrm{M}_{23} \mathrm{C}_{6}$ carbides can be easily distinguished. Because there was almost no vanadium in the steel, the Ta rich precipitates did not give vanadium signal. The maps are shown from two different FIB foils.

For O series steel, BF-TEM and STEM images are presented in Fig. 57. In this steel, both TEM and STEM analysis suggested very less fraction of precipitates. No Ta rich precipitates, giving bright contrast in HAADF images were detectable. Upon STEM-EDX mapping, only a few $\mathrm{Cr}, \mathrm{W}$ rich $\mathrm{M}_{23} \mathrm{C}_{6}$ type particles were detected from two different samples (see Figs. 58-59). The microstructure contained V, N rich precipitates, preferentially along the grain/lath boundaries. We believe these are the MX precipitates, however mostly without Ta. This is expected because the Ta content of this steel was very low (0.05 wt.\%).
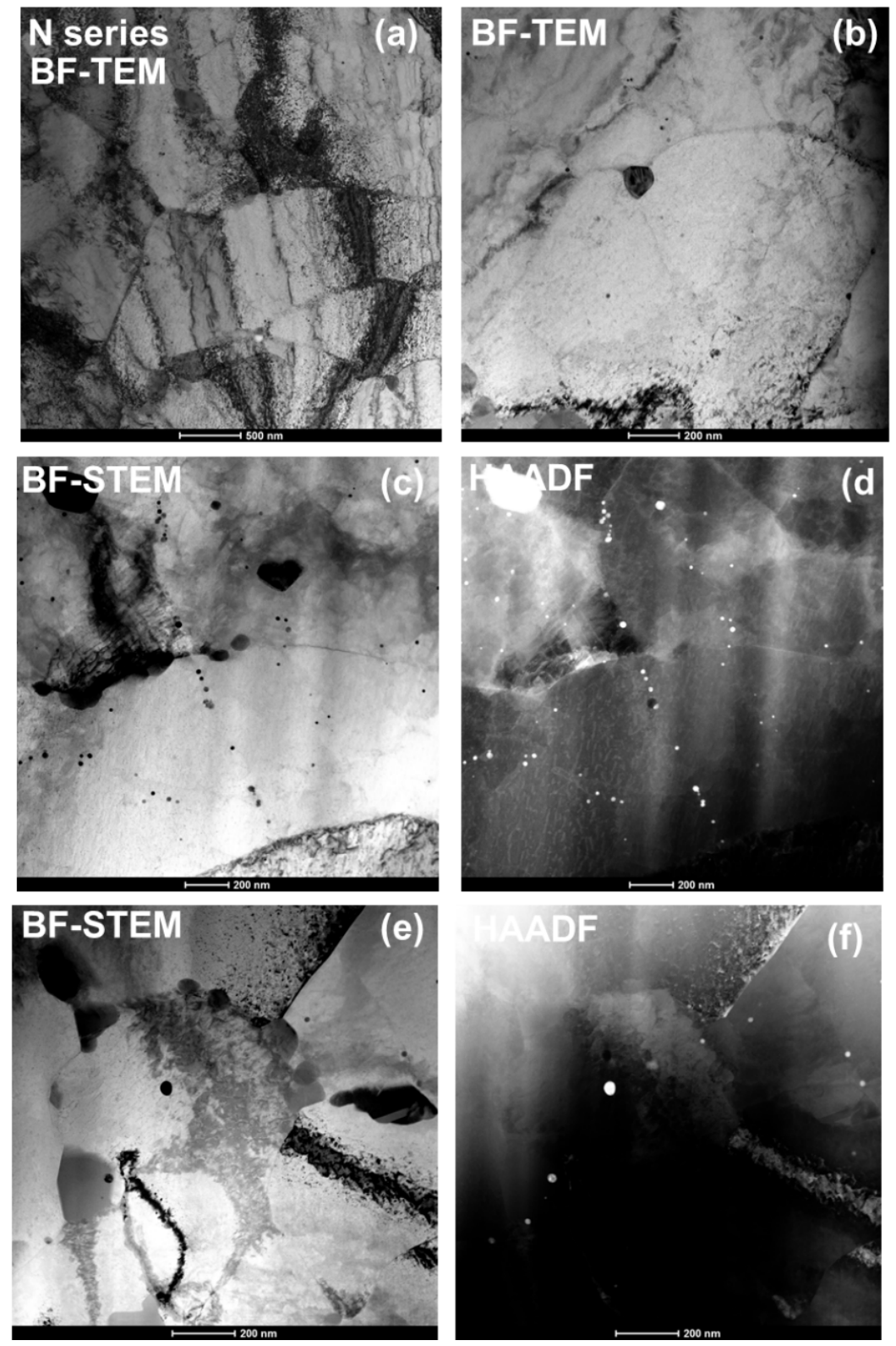

Fig. 55. TEM and STEM analysis of $\mathrm{N}$ series steel. (a,b) BF TEM images. (c, d) BF-STEM and HAADF of same zone. (e,f) BF-STEM and HAADF of same zone, different sample. 


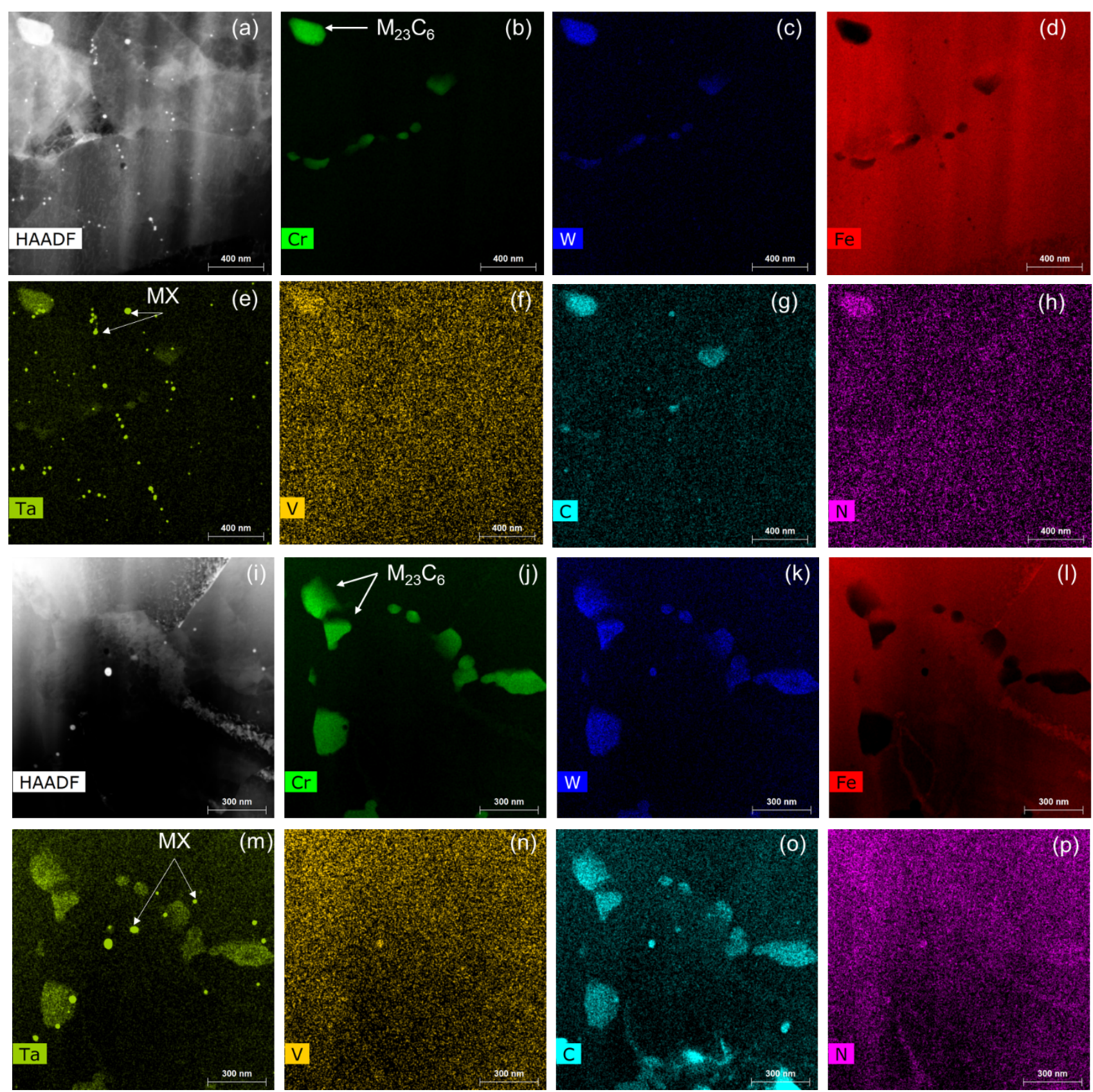

Fig. 56. STEM-EDX mapping of N series steel (a-h) HAADF image and EDX maps of same zone. (i-p) HAADF image and EDX mapping from a different FIB foil. 


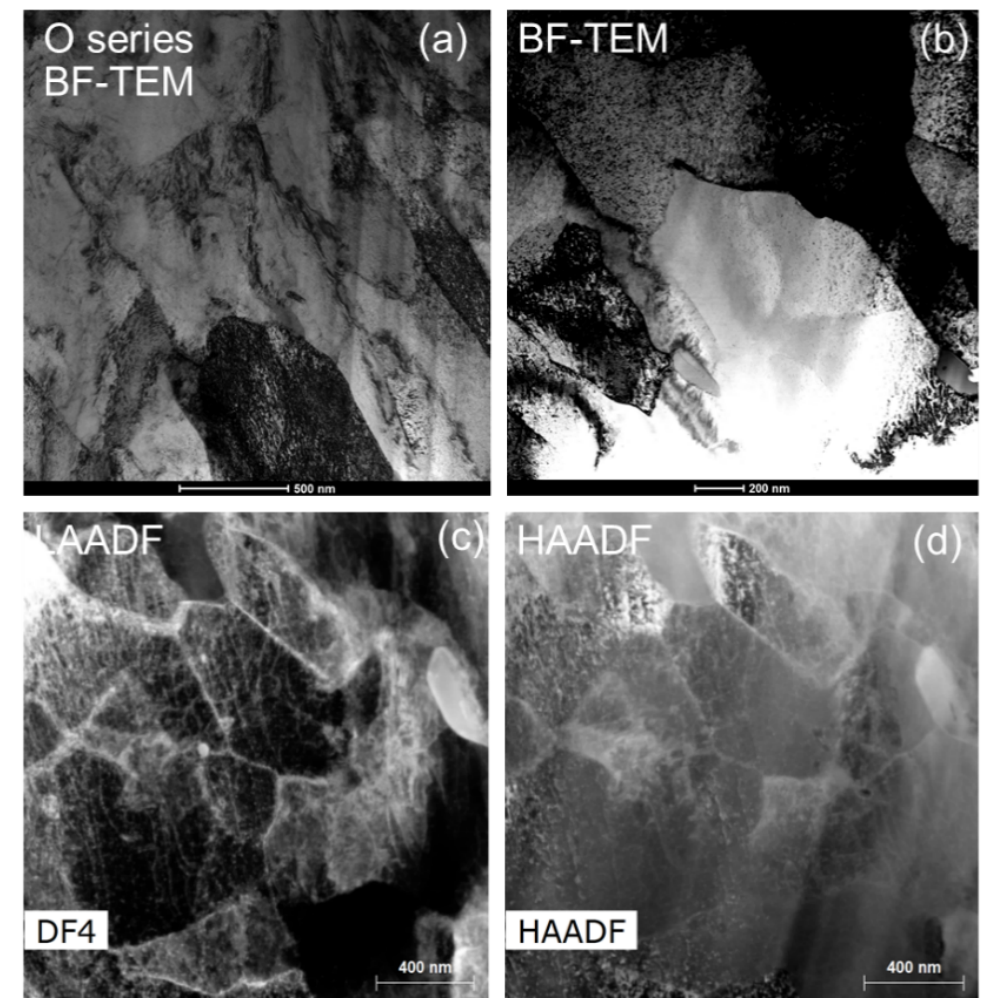

Fig. 57. TEM and STEM analysis of O series steel. (a,b) BF TEM images. (c, d) STEM-LAADF and HAADF images of same zone.
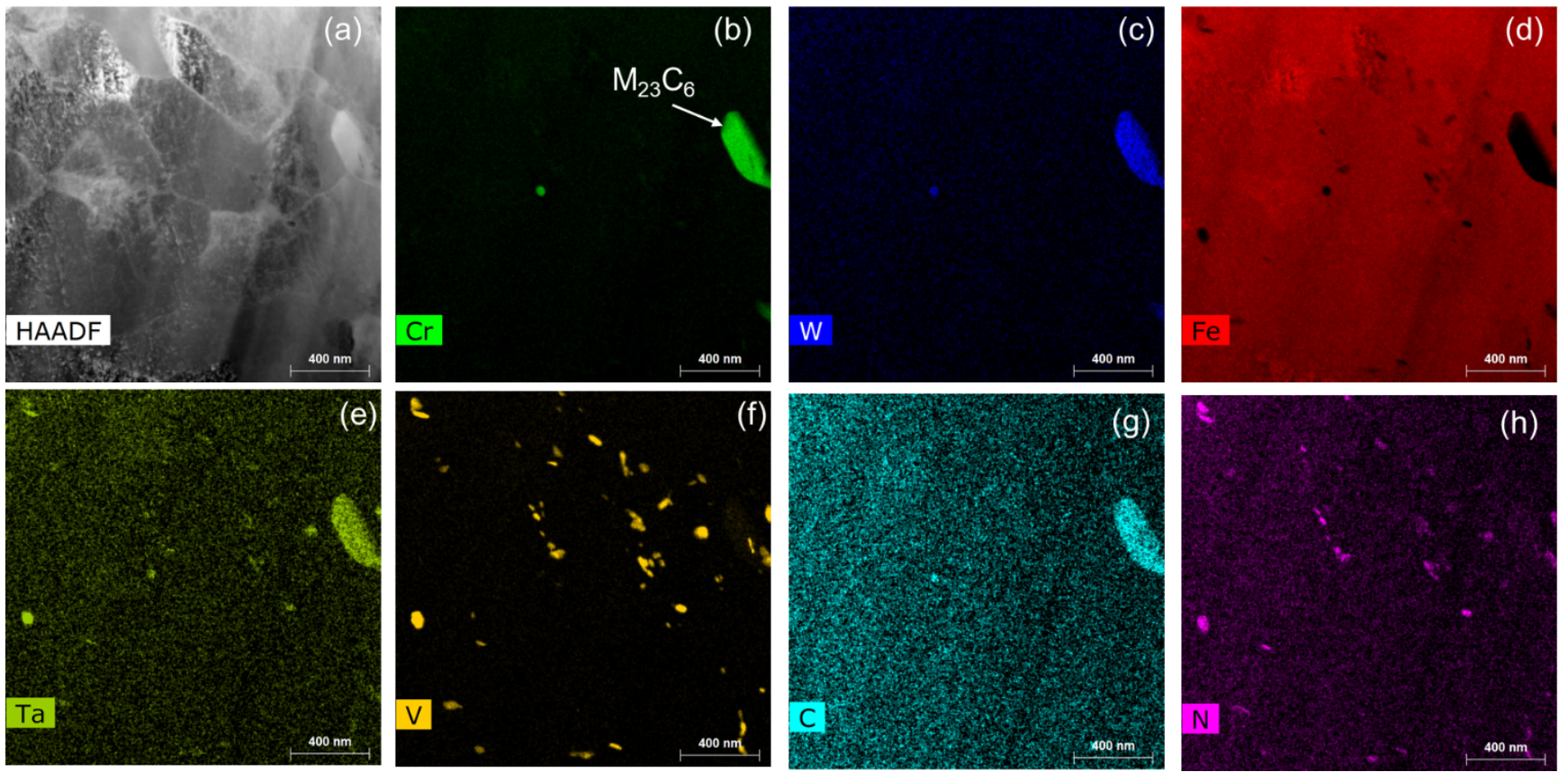

Fig. 58. STEM-EDX mapping of O series steel (a) HAADF image (b-h) EDX maps of same zone. 

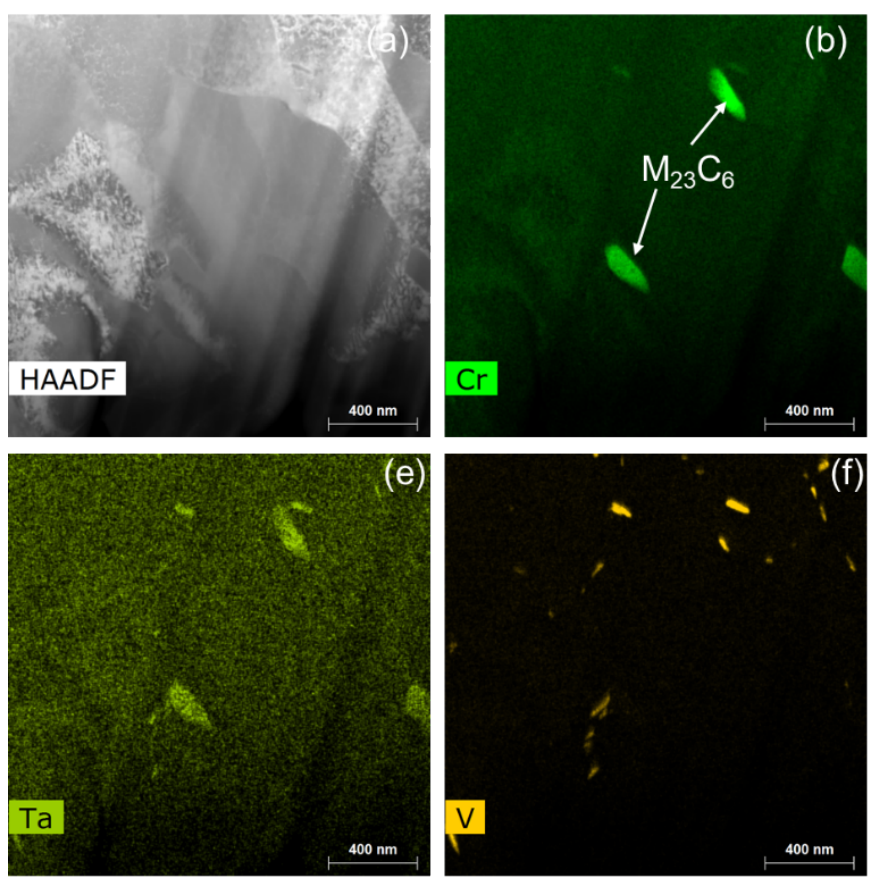
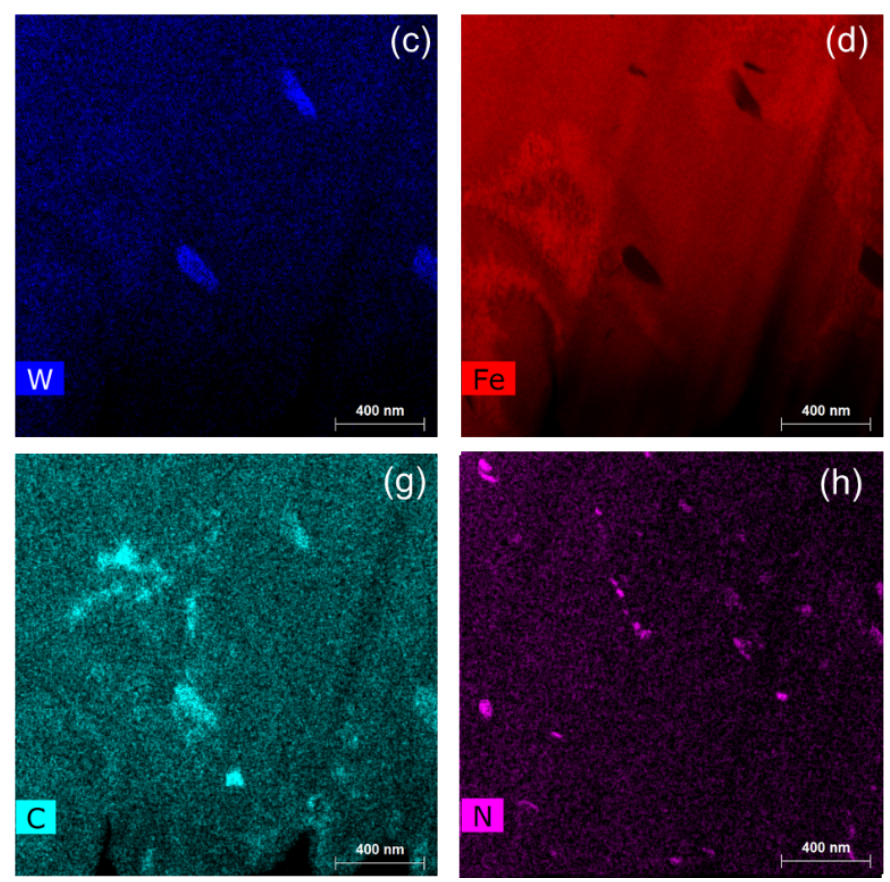

Fig. 59. STEM-EDX mapping of O series steel (a) HAADF image (b-h) EDX maps of same zone, different FIB foil.

\subsubsection{Reference E series steel: KIT}

Fig. 60 presents the optical image of the new reference Eurofer-97 E series steel. The microstructure appeared typical of a tempered F-M steel with laths and precipitates decorating the laths when etched using Villela's reagent. However, some precipitates seemed to be quite coarse (encircled in Fig. 60). To further confirm the presence of coarse precipitates, the steel was polished and then etched using $2 \%$ Nital solution. Following this, the optical images are presented in Fig. 61. These images revealed inhomogeneously distributed network of very coarse precipitates, greater than $1 \mu \mathrm{m}$ in size, in the steel. To the best of our knowledge, such coarse precipitates are uncharacteristic of Eurofer-97 steel grade. These precipitates being very large, are unlikely to be intercepted in FIB foils for TEM analysis, and hence are easy to miss if only TEM is performed.

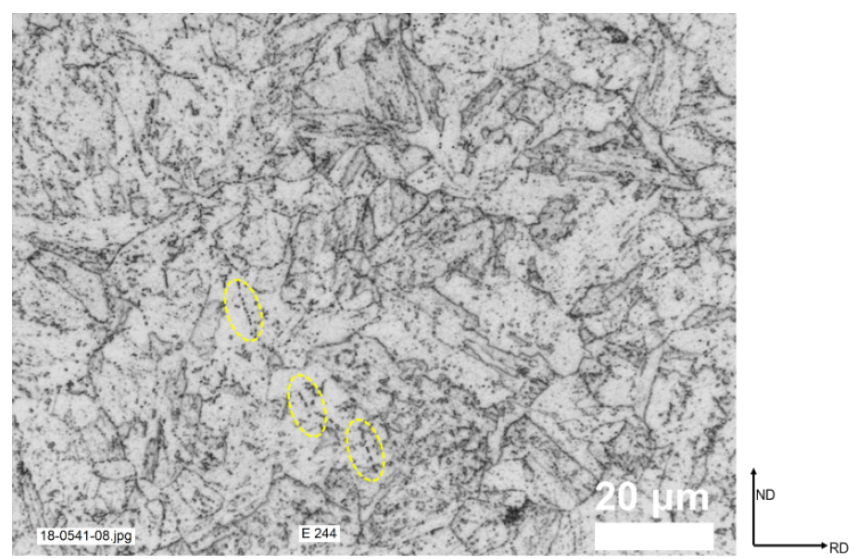

Fig. 60. LOM image of E series steel after etching in Vilella's reagent. Yellow circles show coarse precipitate networks. 

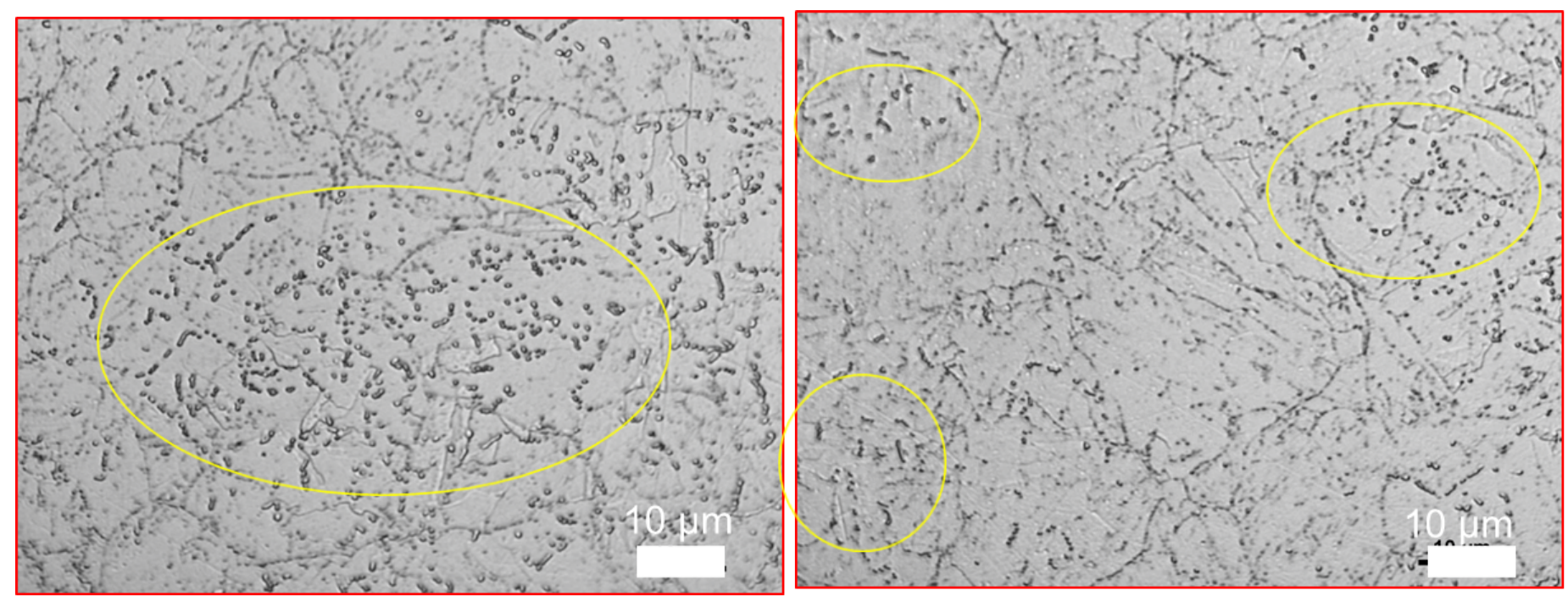

Fig. 61. LOM image of E series steel after etching in 2\% Nital, revealing coarse precipitate networks.

To further verify the presence of such coarse precipitates, SEM imaging was performed on the etched sample. For this analysis, relatively deep etching was performed as compared to previous steels to better reveal the large precipitates without any ambiguities. A sequence of secondary electron SEM images are presented in Fig. 62 where many extremely coarse precipitates, a few microns in size, and networks of such precipitates were identified. These precipitates, which are expected to be coarse carbides, were distributed all over the sample, but usually existing in dense clusters as was also noted by LOM. Such large carbides are unexpected in any 9\% Cr F-M steel under conventional heat treatment condition of 960-980 ${ }^{\circ} \mathrm{C}$ normalization and $750-760{ }^{\circ} \mathrm{C}$ tempering [3]. To give a feeling of how coarse the carbides were when compared to RAFM steels, an SEM image of this steel is placed adjacent to same magnification image obtained from the M series steel in Fig. 63.

Both LOM and SEM analysis, revealing extremely large carbides in the steel, provide a reason for the relatively poor fracture toughness properties of as-received E series steels obtained using bend bar samples, and further confirm that the steel properties/microstructure do not confer well with rather wellknown properties/microstructure of a Eurofer-97 steel grade. This steel was an improvement over the previously provided G/F series reference Eurofer-97 steel, the microstructure/properties of which are provided in the appendix. Compared to the $\mathrm{G}$ series, the microstructure/properties of $\mathrm{E}$ series did show improvement.

TEM analysis of this steel was performed on FIB foils, with precipitates mapped using STEMEDX. Because of very large size of the coarse carbides detected in the optical images, and their inhomogeneous distribution, such carbides were not intercepted in the two FIB foils extracted from this steel. Figs. 64a-64b present STEM-MAADF images showing the distribution of some precipitates in the steel. Judging from their size/morphology, these precipitates are expected to be $\mathrm{M}_{23} \mathrm{C}_{6}$ type. $\mathrm{MX}$ carbides were detected in HAADF images in Figs. 64c-64d where they appeared bright on a relatively dark background. Fig. 65 shows STEM-MAADF, HAADF and EDX elemental map of Cr, Ta from the same zone of analysis. $\mathrm{Cr}$ and Ta maps were superimposed, and these two elements were used to differentiate between $\mathrm{M}_{23} \mathrm{C}_{6}$ and $\mathrm{MX}$ phases in the same image. It is evident that the bright globular precipitates detected in HAADF were Ta rich and will be the MX phase, while the bigger rather facetted carbides were $\mathrm{Cr}$ rich and hence will be the $\mathrm{M}_{23} \mathrm{C}_{6}$ phase (see Fig. 65). Fig. 66 shows EDX maps of precipitates in this steel from a second FIB foil (compared to Fig. 65), extracted from a different tensile sample. No significantly coarse carbides, as seen by LOM/SEM, were detectable in this foil as well, which we attribute to the much smaller areas of analysis in the FIB foils. These results also highlight that often FIB-TEM analysis is not representative of a material due to much smaller areas of analysis. This is particularly a problem for rather inhomogeneous materials in terms of precipitate distribution such as RAFM steels. 

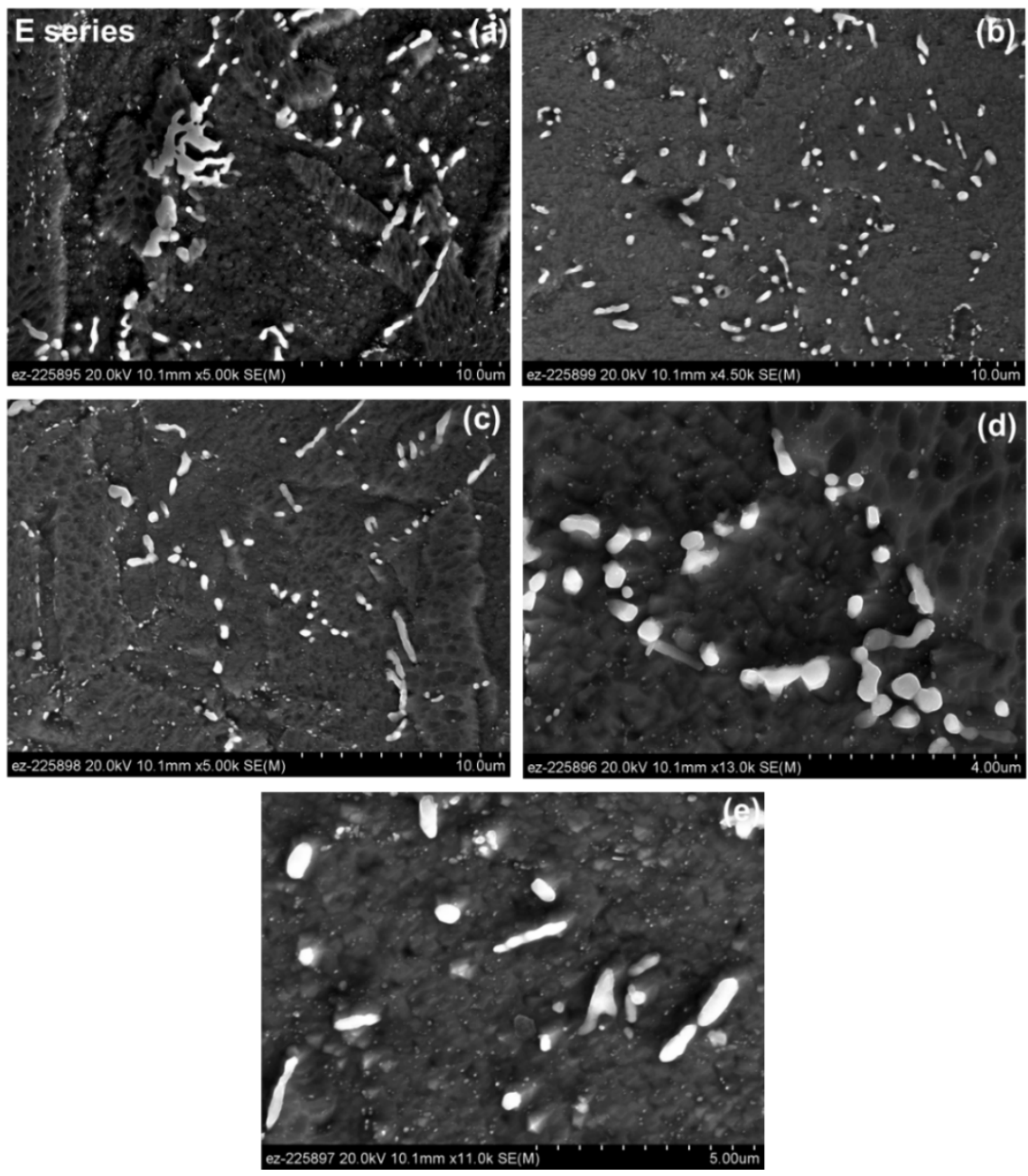

Fig. 62. SEM secondary electron images of very coarse precipitates detected in reference E series Eurofer-97 steel.
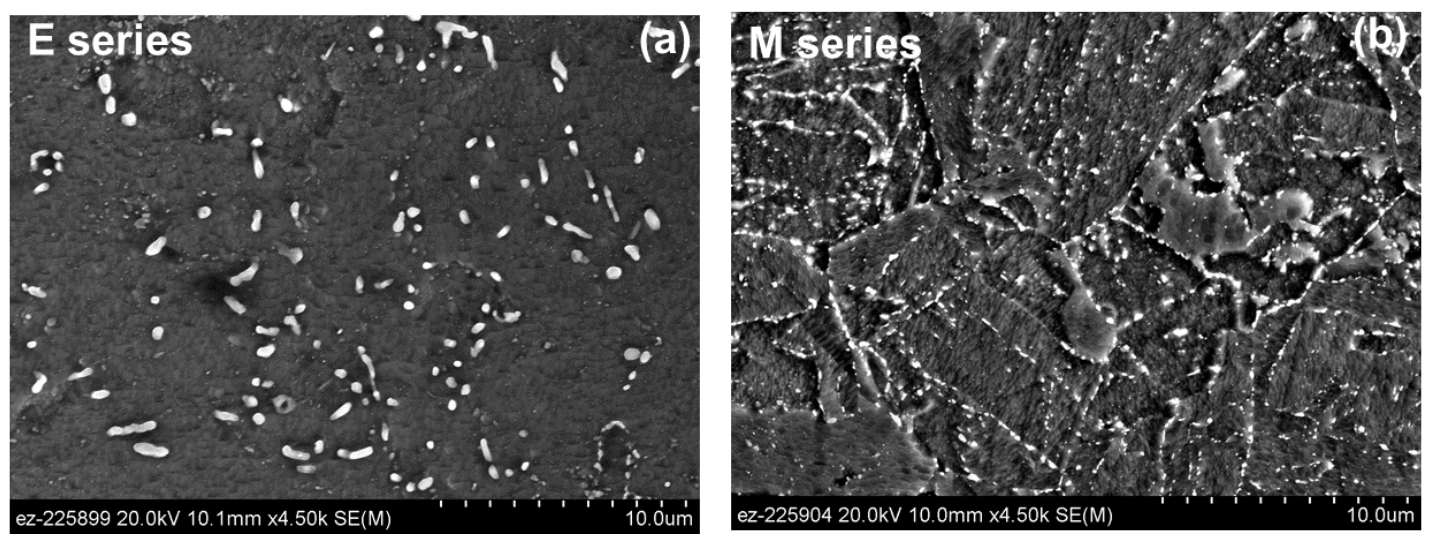

Fig. 63. SEM secondary electron images obtained at same magnification comparing reference E series with $\mathrm{M}$ series steel, to highlight the size of coarse carbides in E series. 


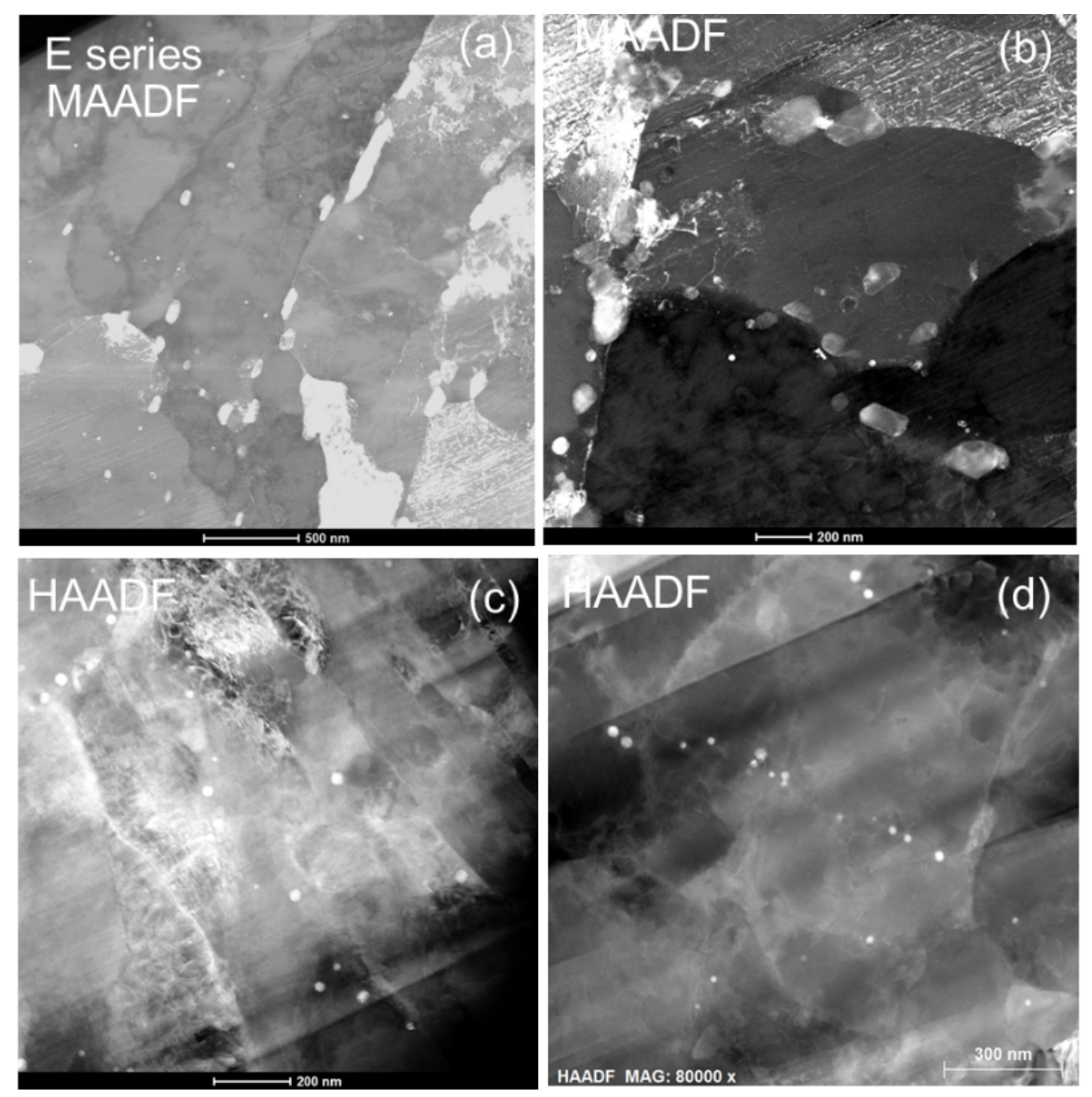

Fig. 64. STEM imaging of E series steel. (a, b) MAADF images showing large carbides: (a) and (b) are from two different FIB foils. (c, d) HAADF images showing MX carbides.
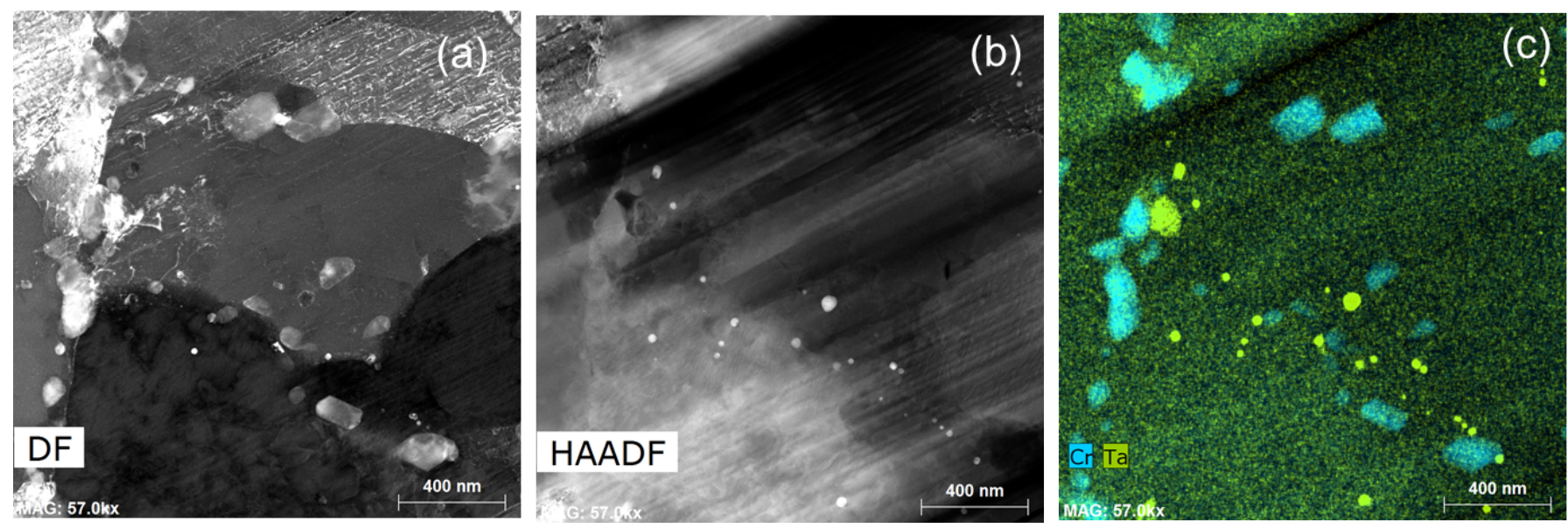

Fig. 65. STEM imaging and EDX mapping of E series steel. (a) MAADF image (b) HAADF image (c) $\mathrm{Cr}$ and Ta EDX elemental map. Ta rich particles will be the MX phase while $\mathrm{Cr}$ rich coarse precipitates are expected to be the $\mathrm{M}_{23} \mathrm{C}_{6}$ phase. 

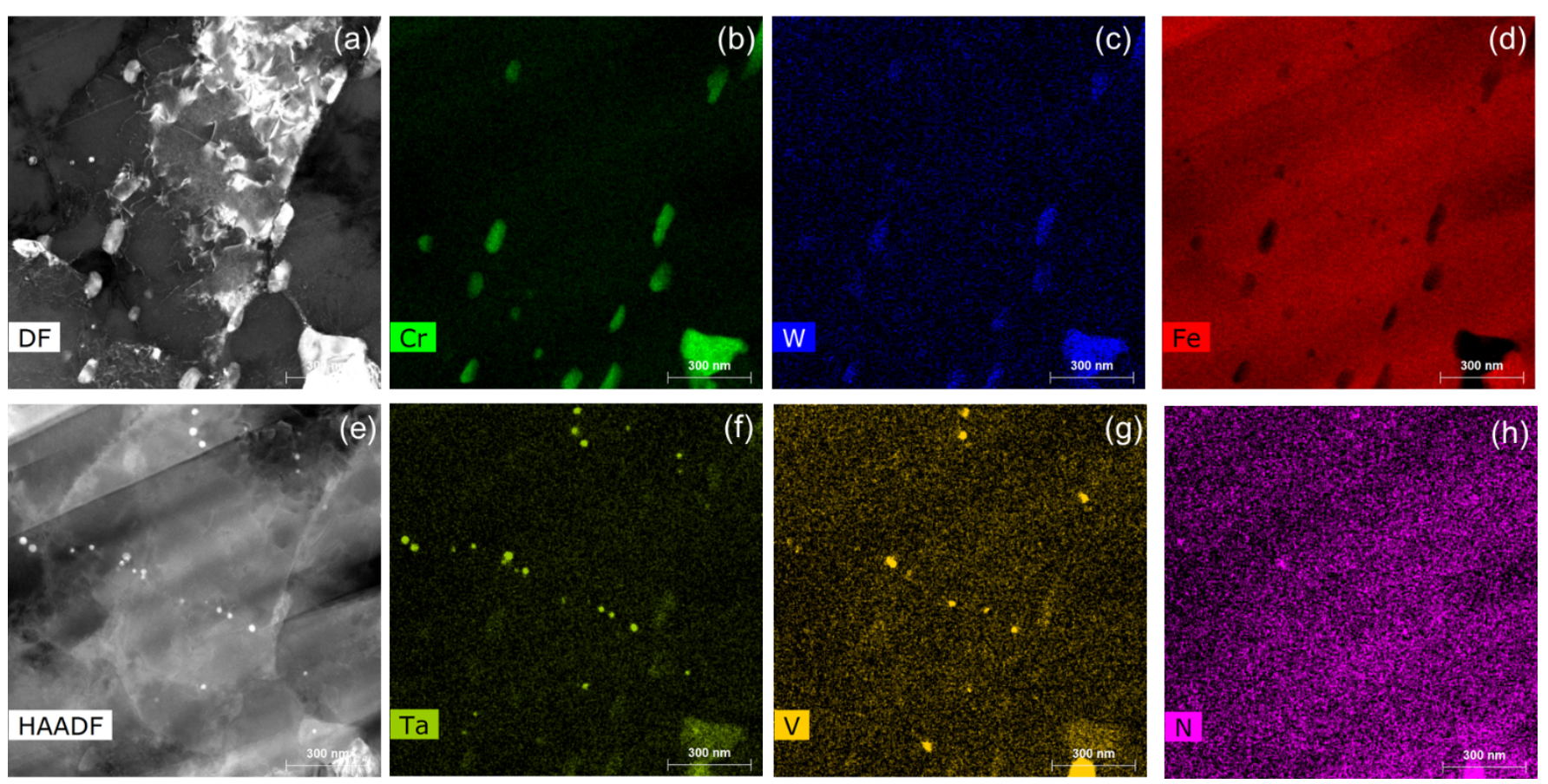

Fig. 66. STEM imaging and EDX mapping of E series steel: same zone of analysis, second FIB foil.

Fractography of the E series steel after room temperature tensile tests was also performed using SEM. Fig. 67a presents the overall fracture surface of the sample. Fig. $67 \mathrm{~b}$ presents a relatively high magnification image of the fracture surface showing the ductile cup and cone type features. No areas of brittle cleavages were identified on the fracture surface.

We must note that the sizes of "holes" in the cup and cone fracture surface of the E series steel appeared rather large, which is also indicative of coarse particles in the steel. This is conclusive when the fracture surface of the reference $\mathrm{E}$ steel and $\mathrm{M}$ series steel are compared under similar magnifications in Fig. 68.
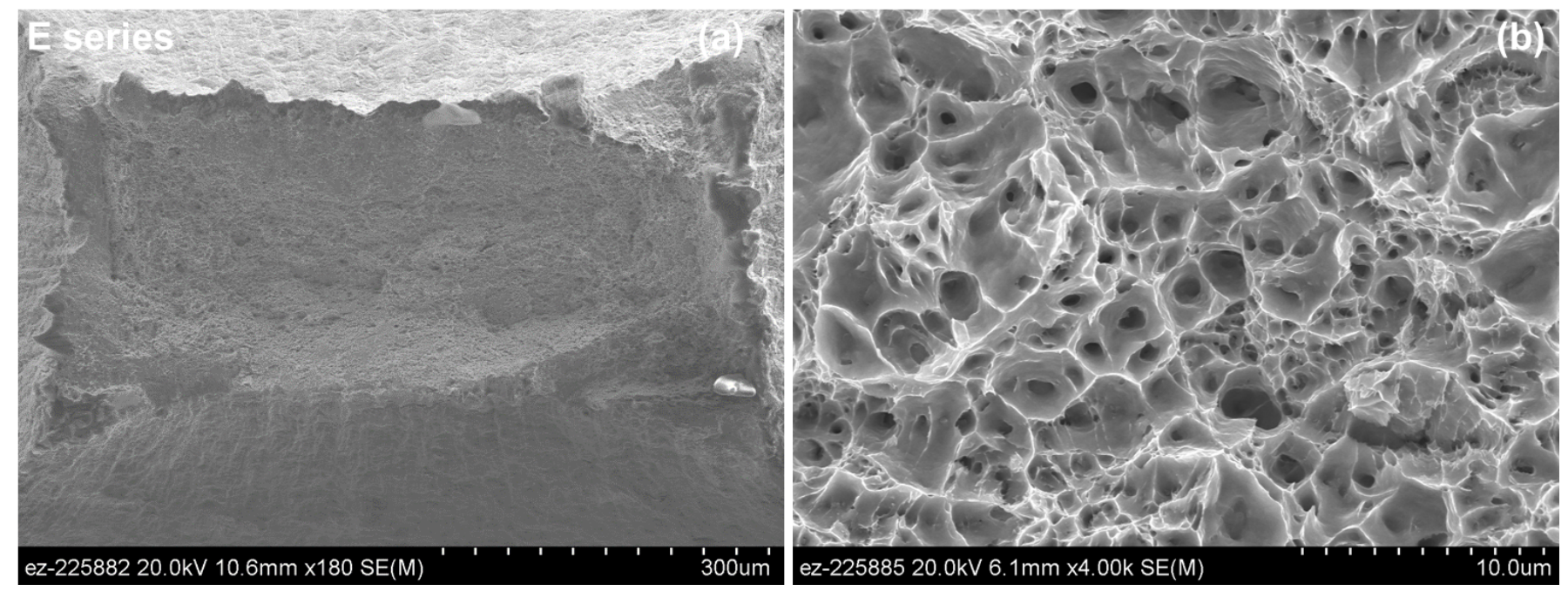

Fig. 67. Fractography of E series steel using SEM secondary electron imaging. (a) Fracture surface overview. (b) Relatively high magnification image showing the ductile cup and cone fracture surface. 

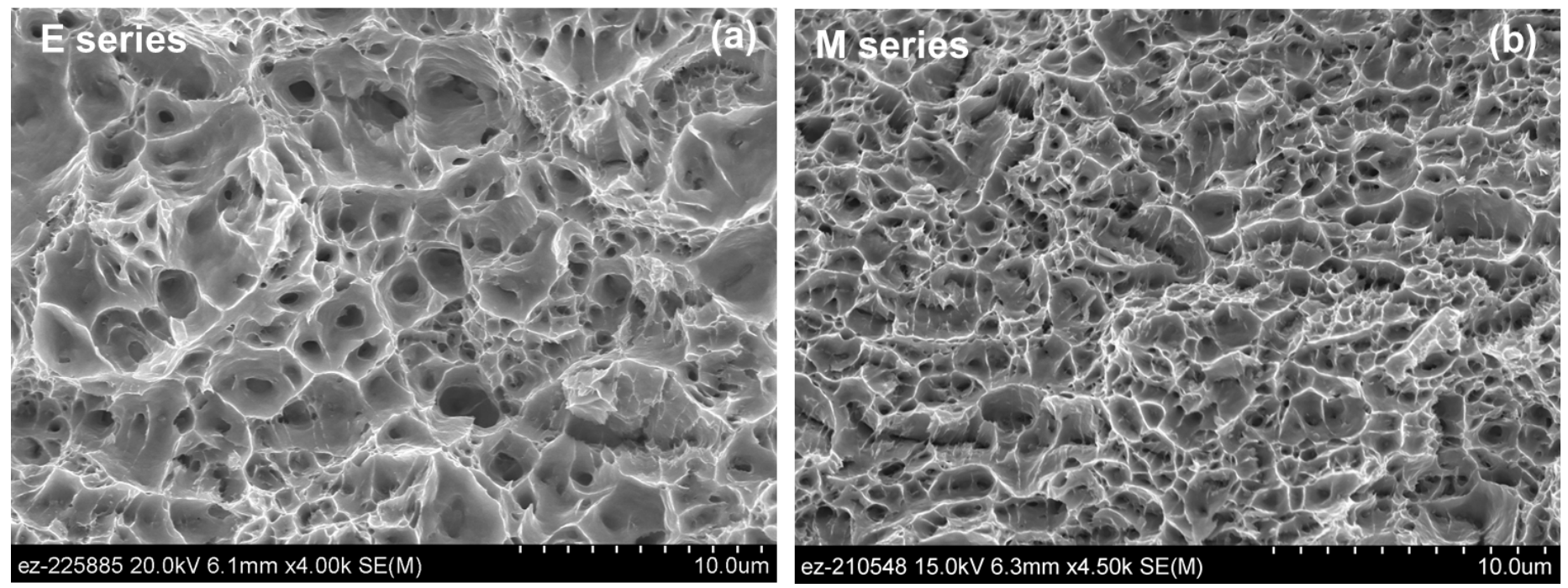

Fig. 68. Comparison of fracture surface of $\mathrm{E}$ series with $\mathrm{M}$ series steels, further proving the presence of coarse particles in the reference material. (a) E series, $x 4 k$ magnification. (b) $M$ series, $x 4.5 \mathrm{k}$ magnification.

\section{References}

[1] ASTM E1921-17a: Standard Test Method for Determination of Reference Temperature, $\mathrm{T}_{0}$, for Ferritic Steels in the Transition Range, ASTM International, West Conshohocken, PA, 2017.

[2] Underwood et al., ASTM STP 1114 (1991) p197.

[3] R. Klueh and D. Harries, High-chromium ferritic and martensitic steels for nuclear applications, ASTM, Bridgeport (2001)

[4] Henry et al. Improvement of Eurofer 97 high-temperature mechanical properties using non-standard heat treatments, 17th International Conference on Fusion Reactor Materials, Aachen, (2015). 


\section{Appendix}

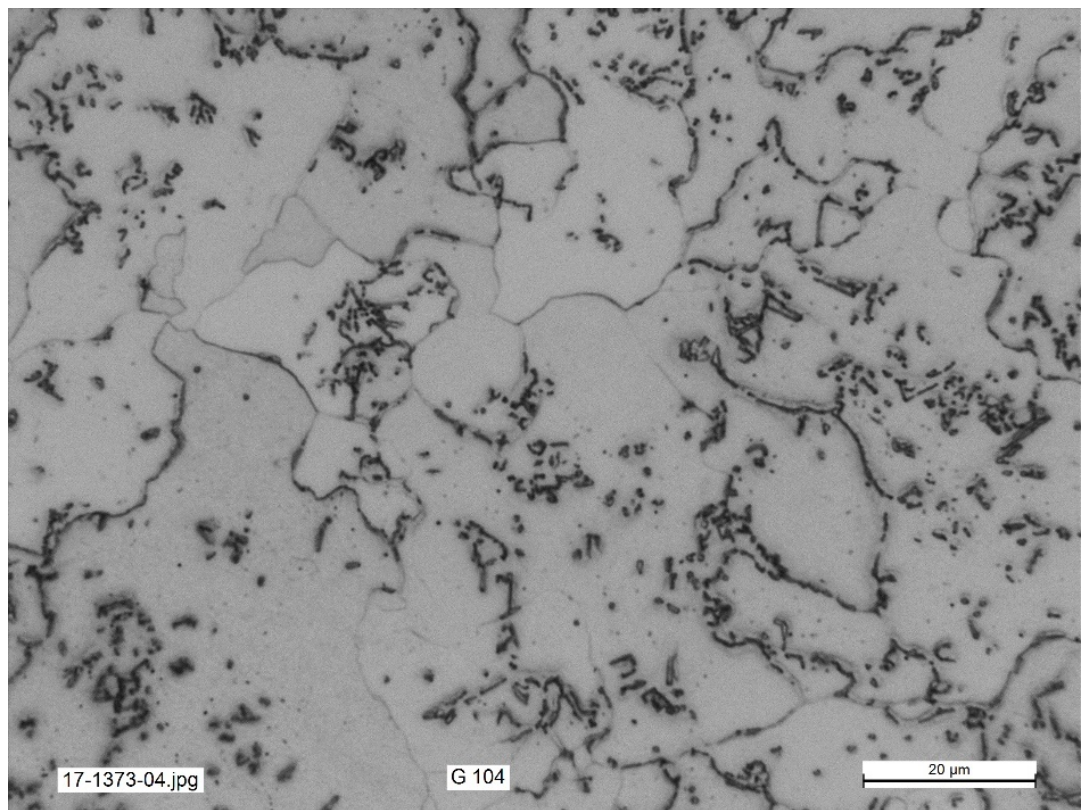

Fig. A1. LOM image of G series steel (old reference) after etching using Villella's reagent. No martensitic laths were detected, and microstructure consisted of very coarse clustered carbides. Grain boundaries were seen to be decorated with films of precipitates.

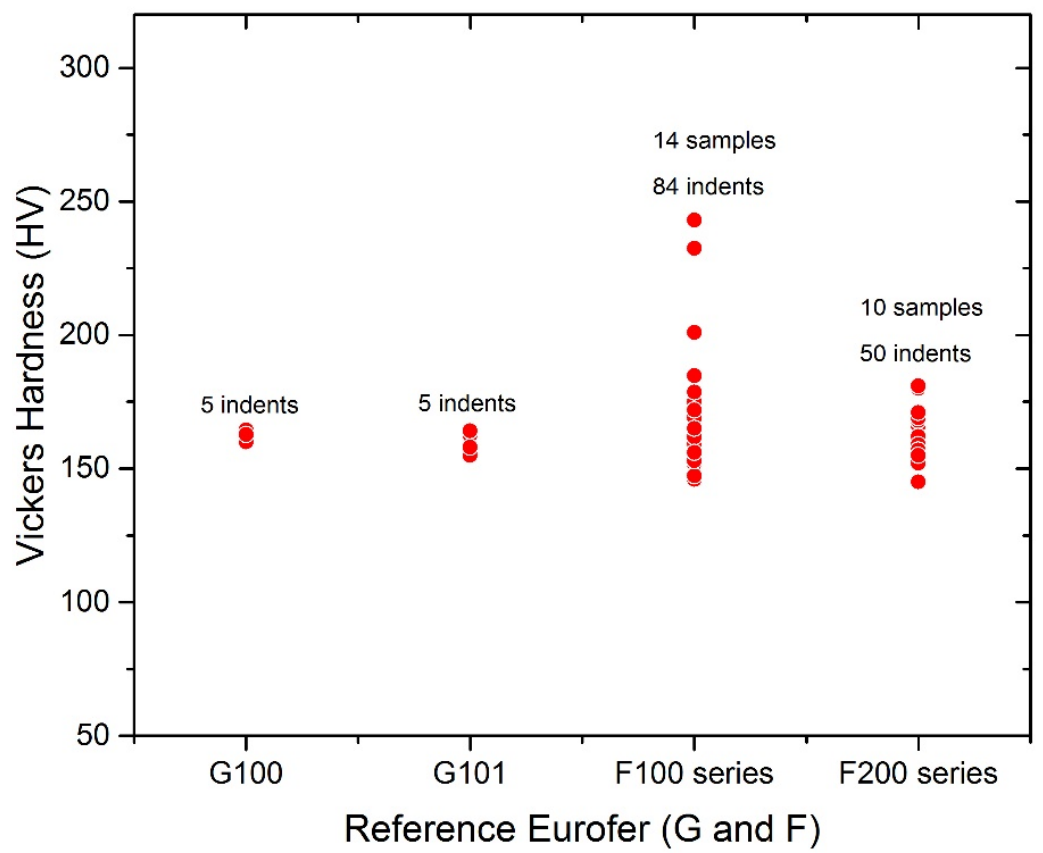

Fig. A2. Vickers hardness indentation results for the reference $G$ and $F$ series steels ( $G$ and $F$ were same steels but different names). $1 \mathrm{~kg}$ load, $15 \mathrm{~s}$ dwell time. These steels were extremely soft. 


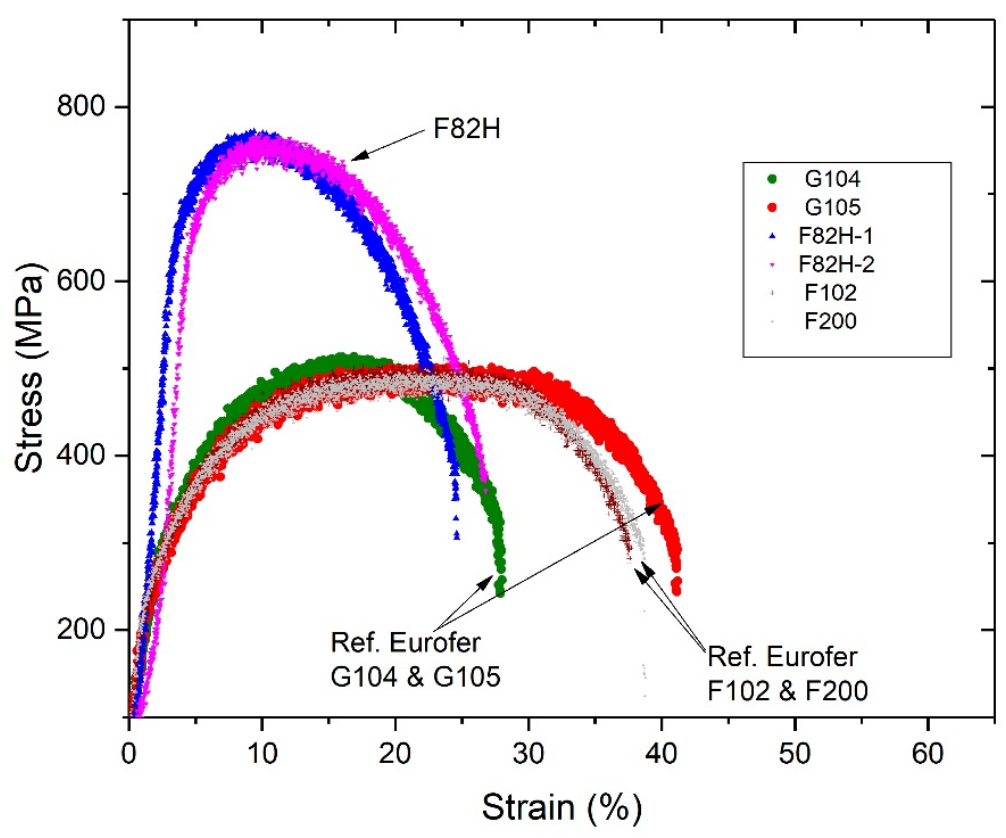

Fig. A3. Comparison of room temperature engineering stress-strain curves for the old reference G/F series Eurofer-97 steels with F82H. G, F were very soft steels. SS-J3 samples.

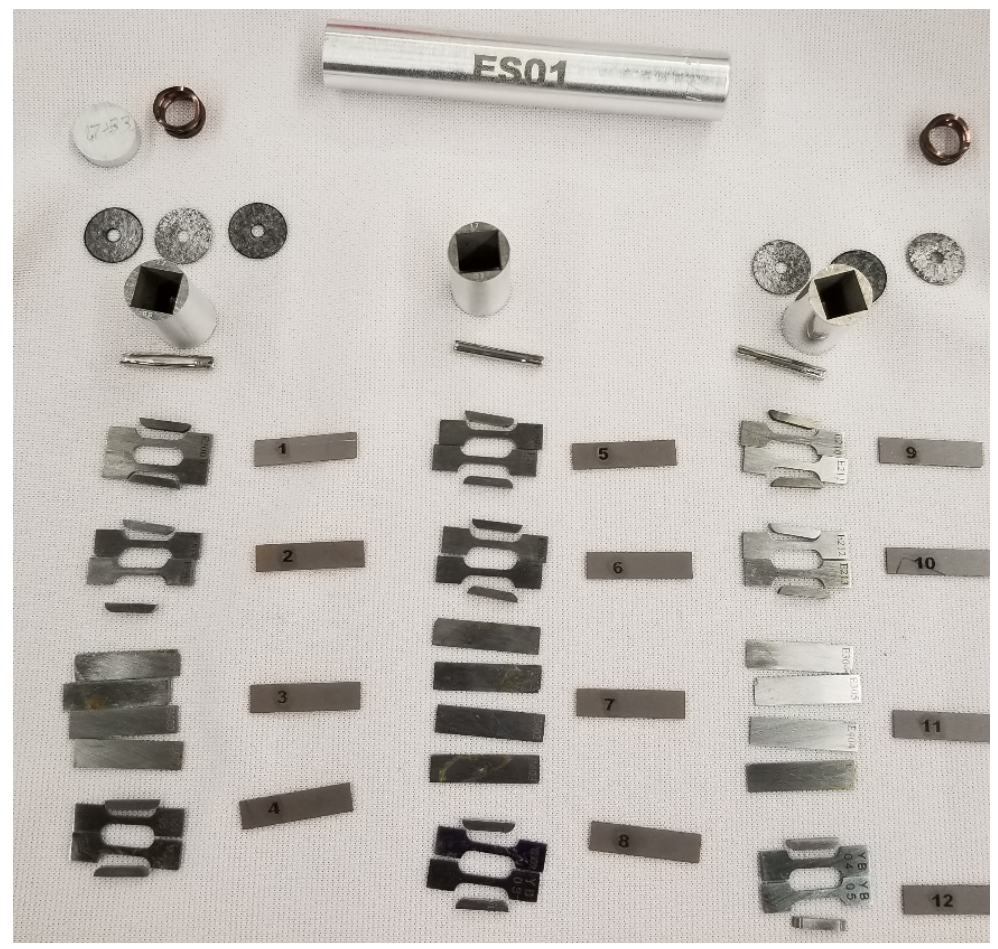

Fig. A4. Parts layout for rabbit ES01. 


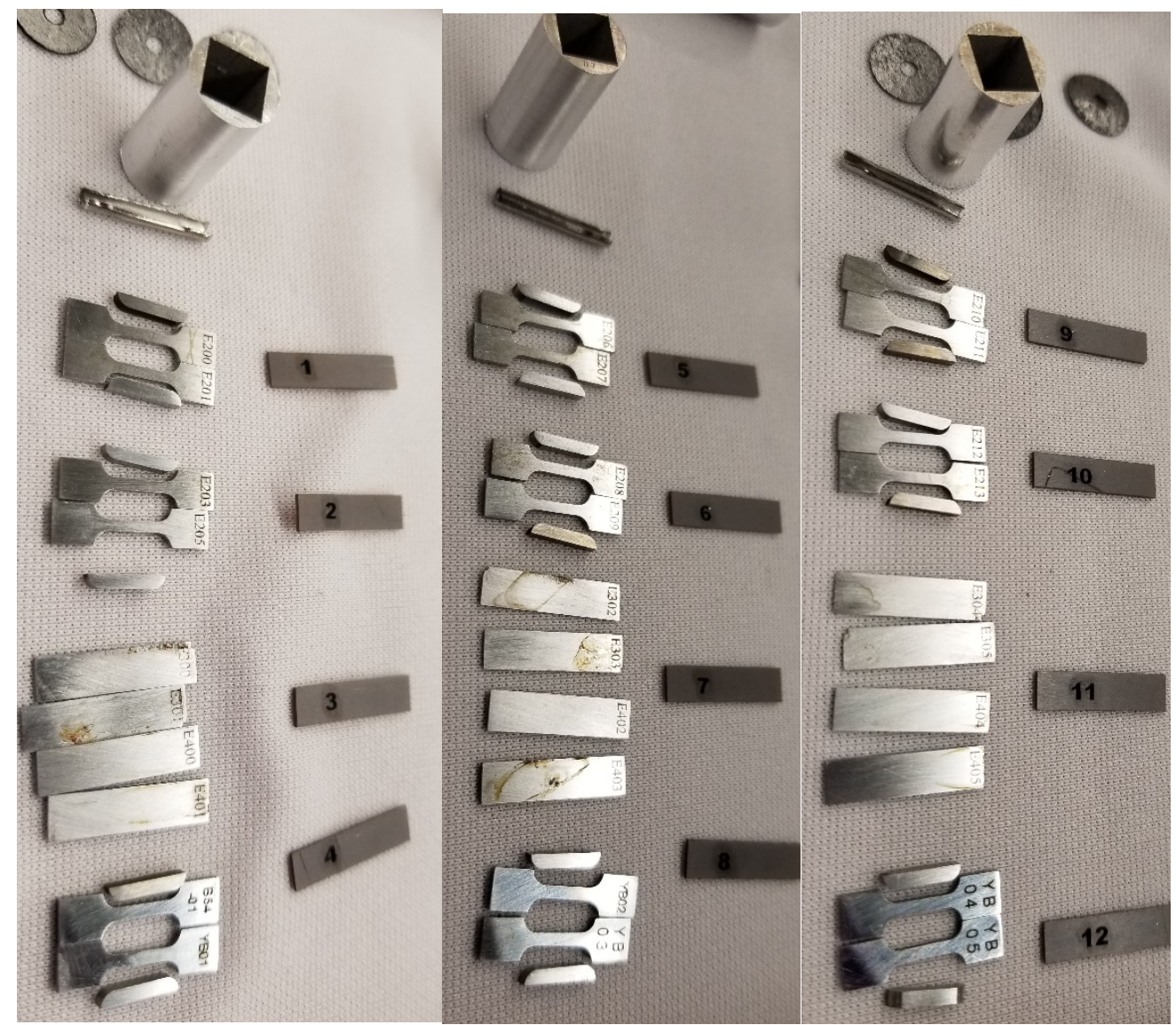

Fig. A5. Parts layout for the three holders of rabbit ES01

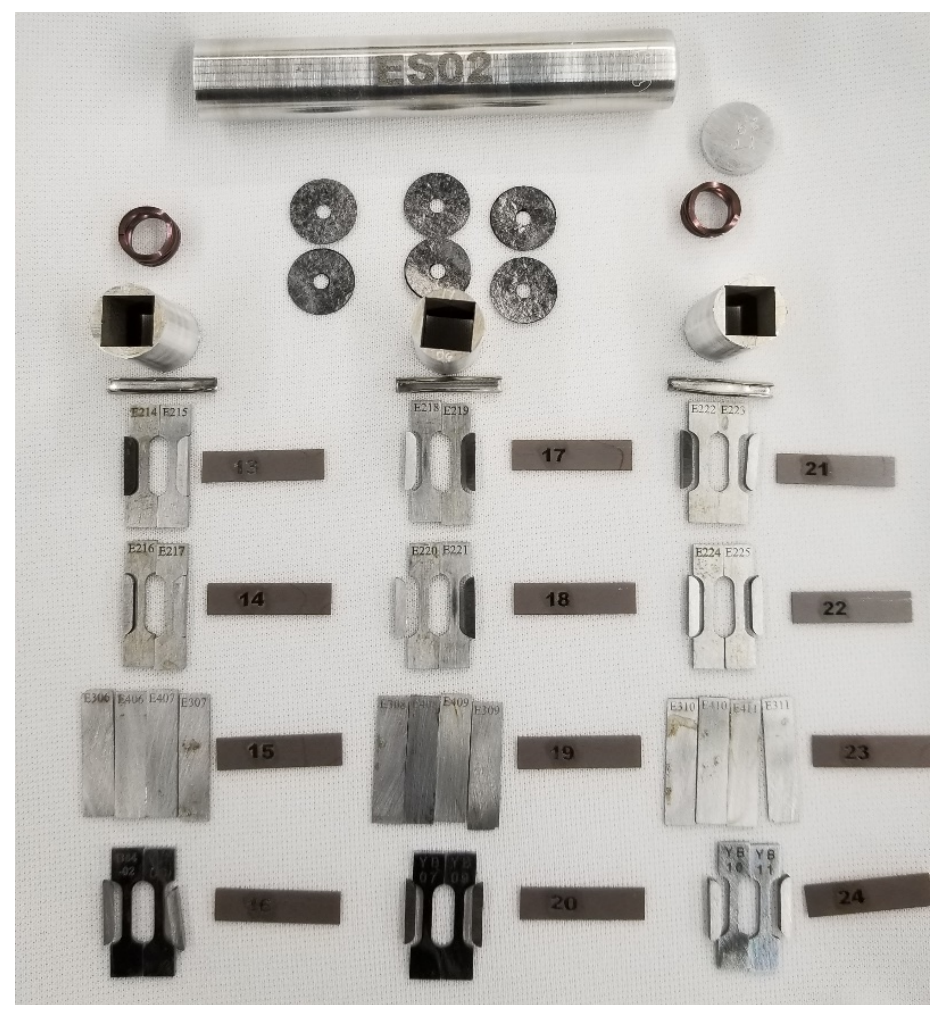

Fig. A6. Parts layout for rabbit ES02. 


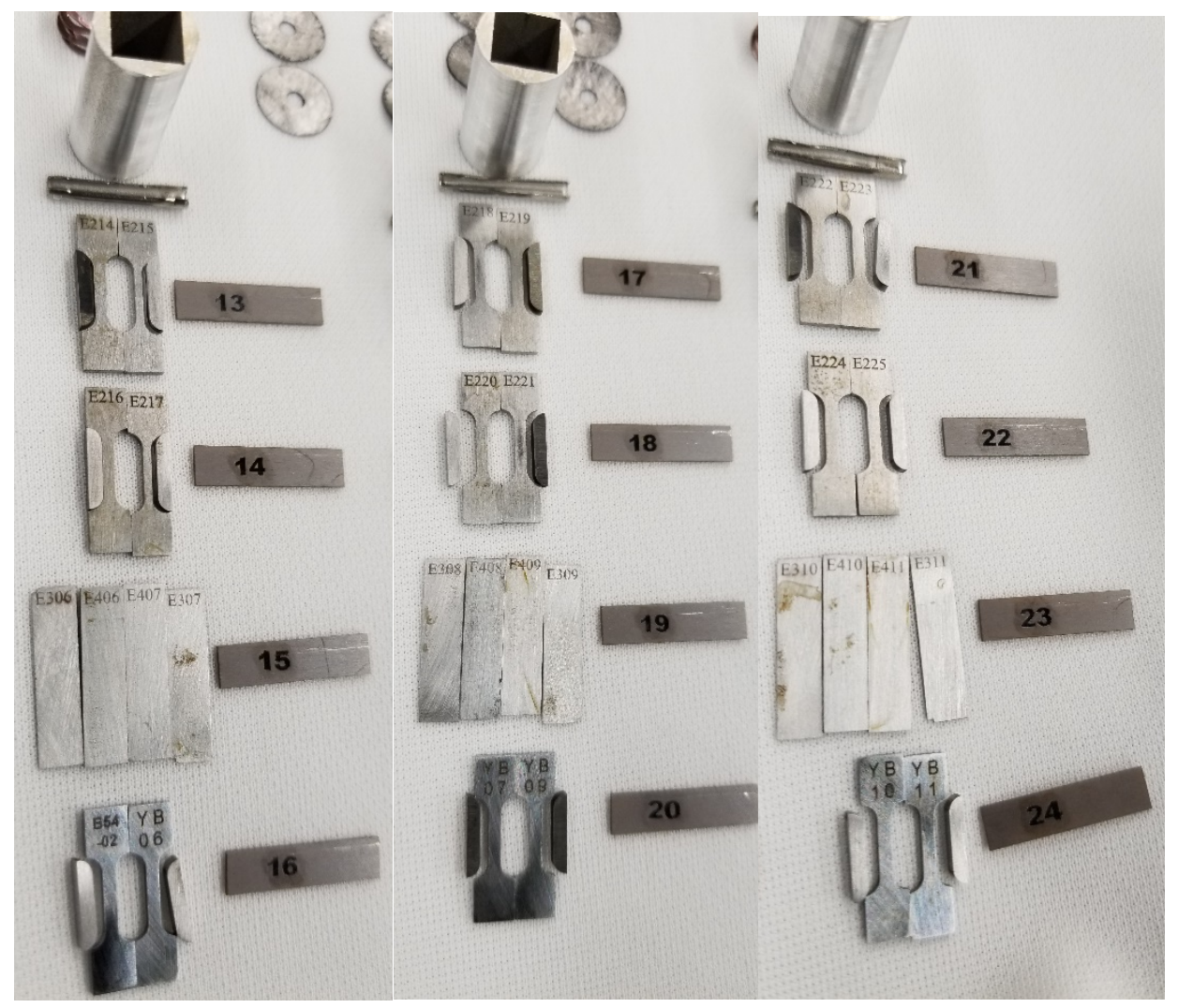

Fig. A7. Parts layout for the three holders of rabbit ES02.

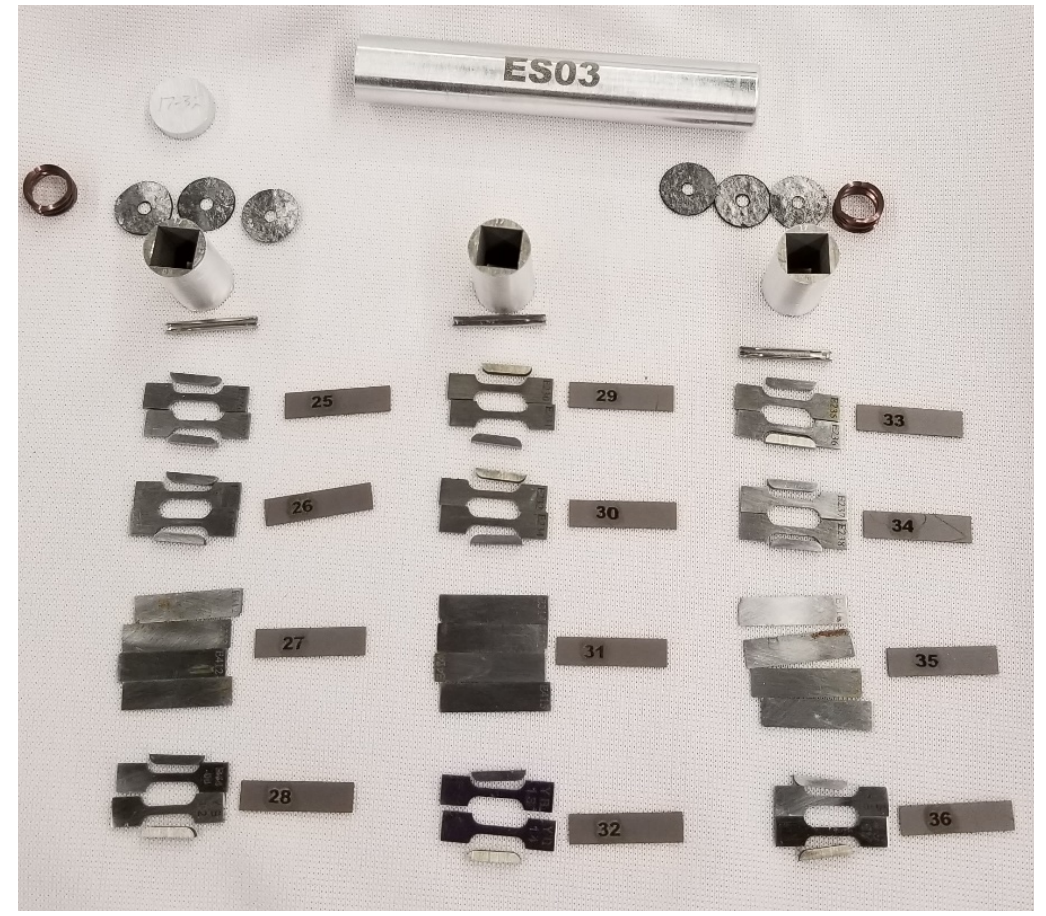

Fig. A8. Parts layout for rabbit ES03. 


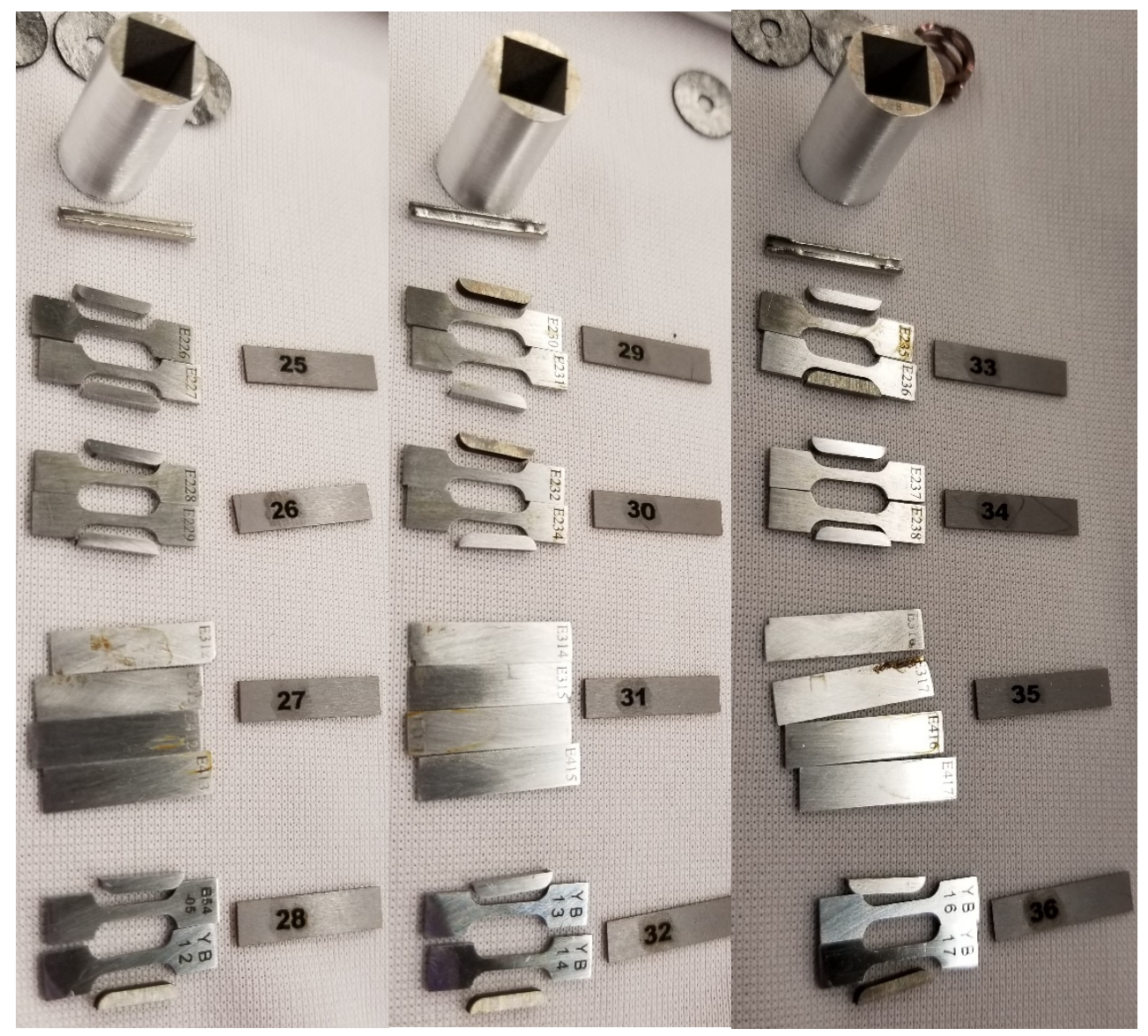

Fig. A9. Parts layout for the three holders of rabbit ES03.

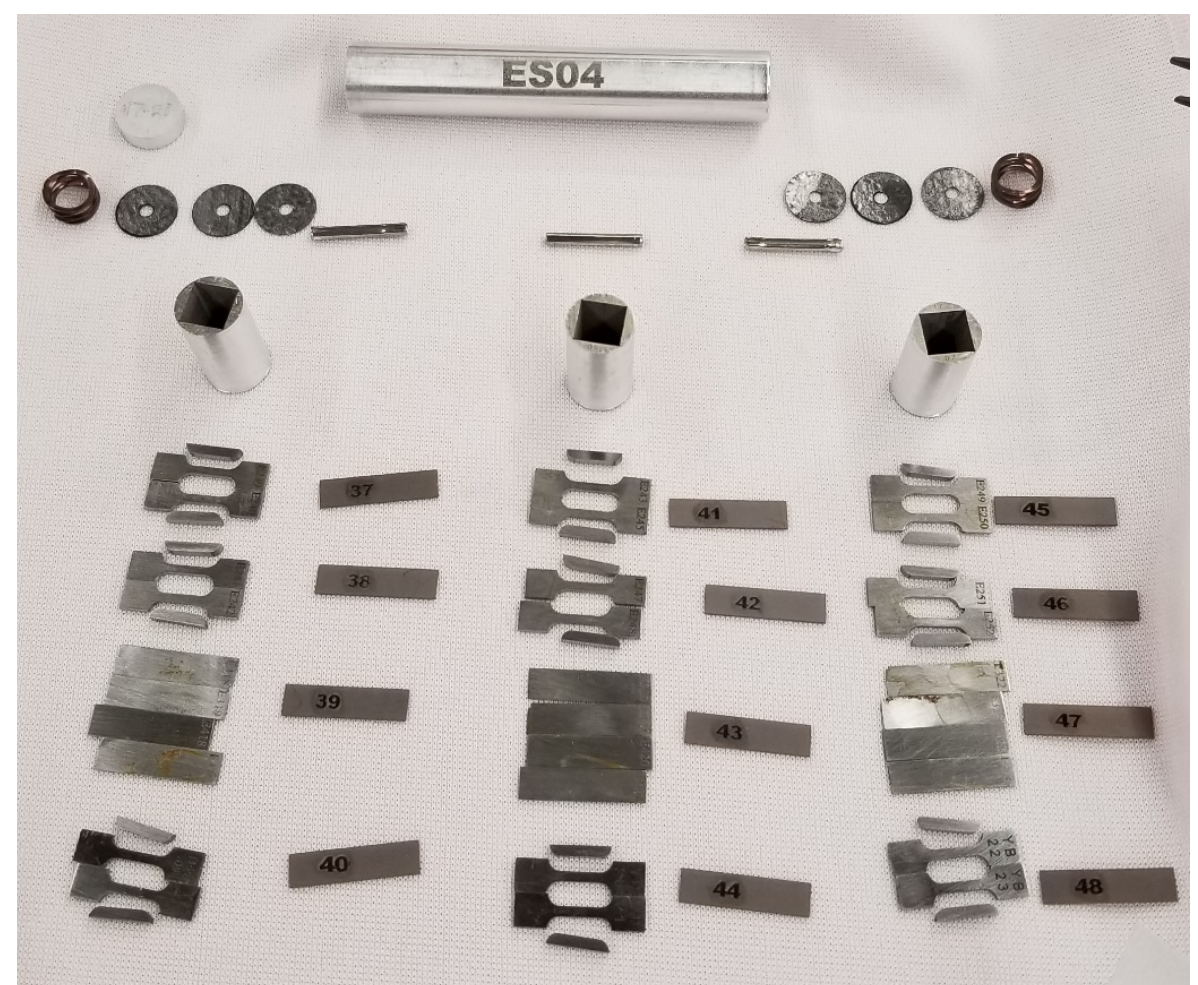

Fig. A10. Parts layout for rabbit ES04. 


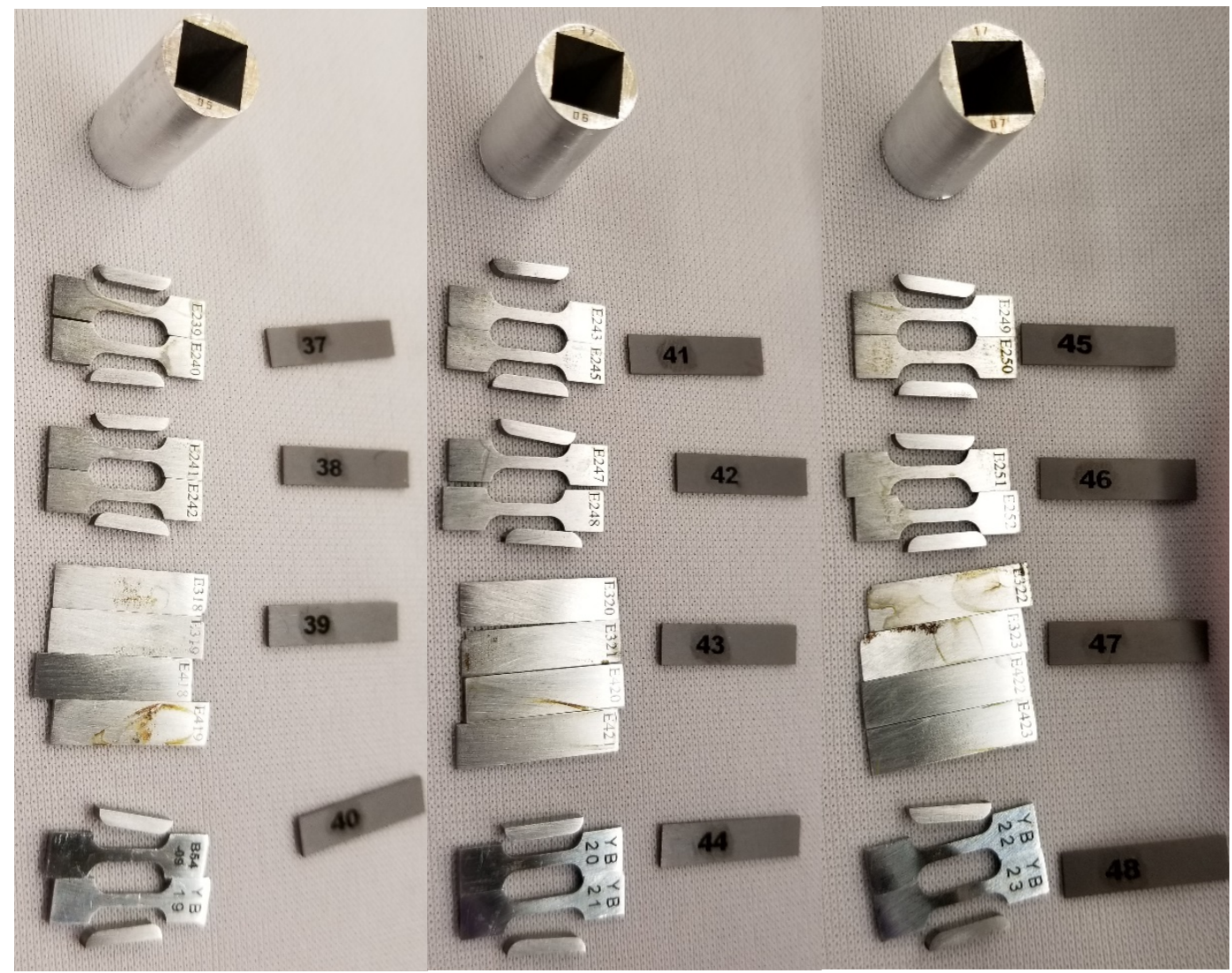

Fig. A11. Parts layout for the three holders of rabbit ES04.

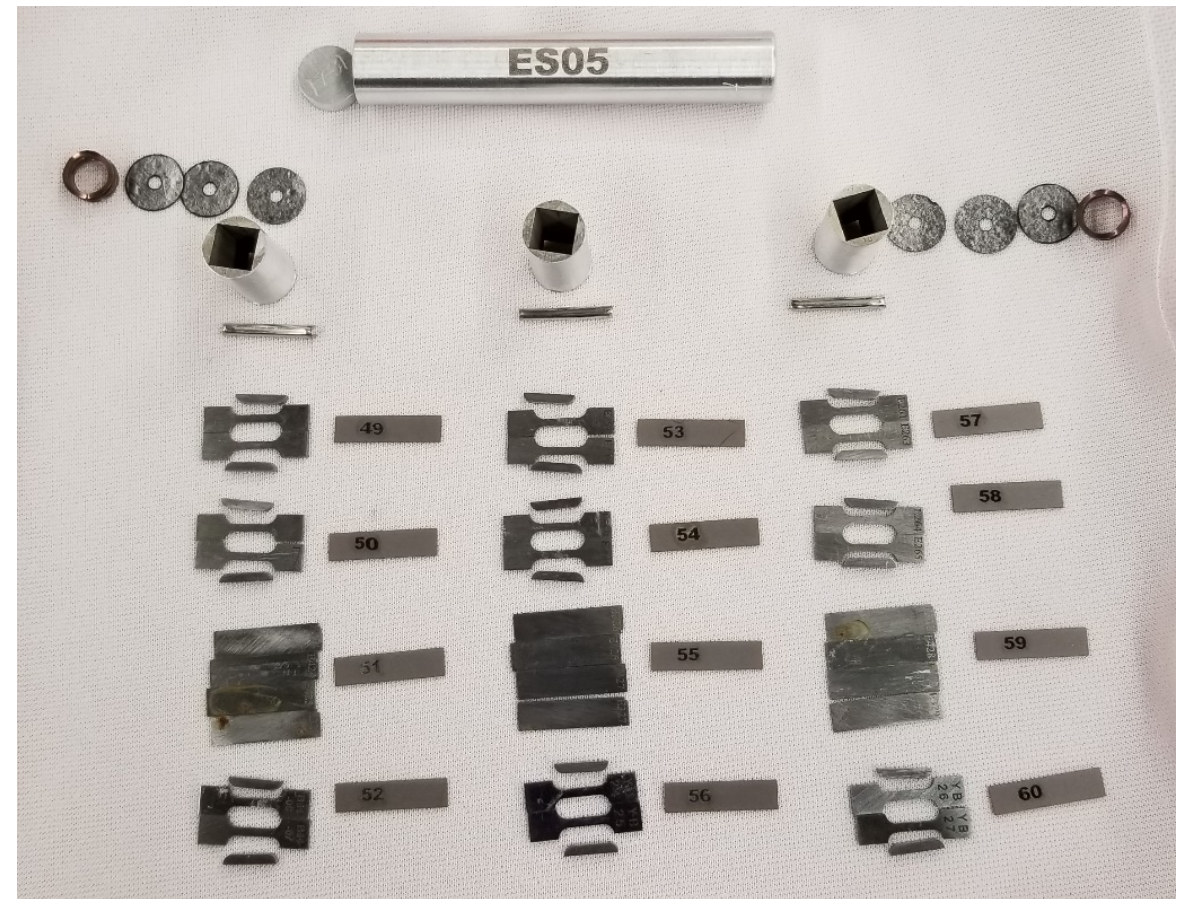

Fig. A12. Parts layout for rabbit ES05. 


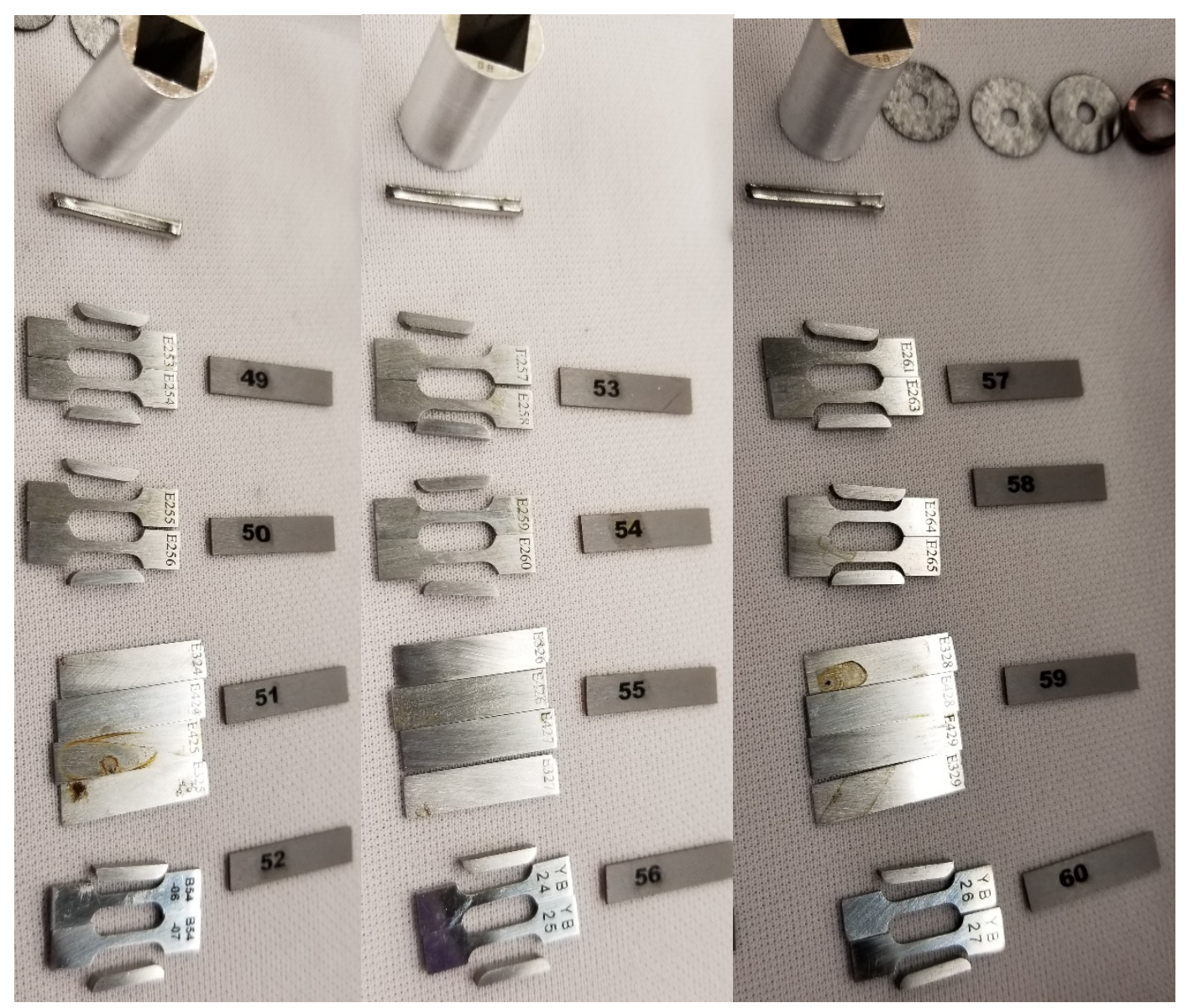

Fig. A13. Parts layout for the three holders of rabbit ES05.

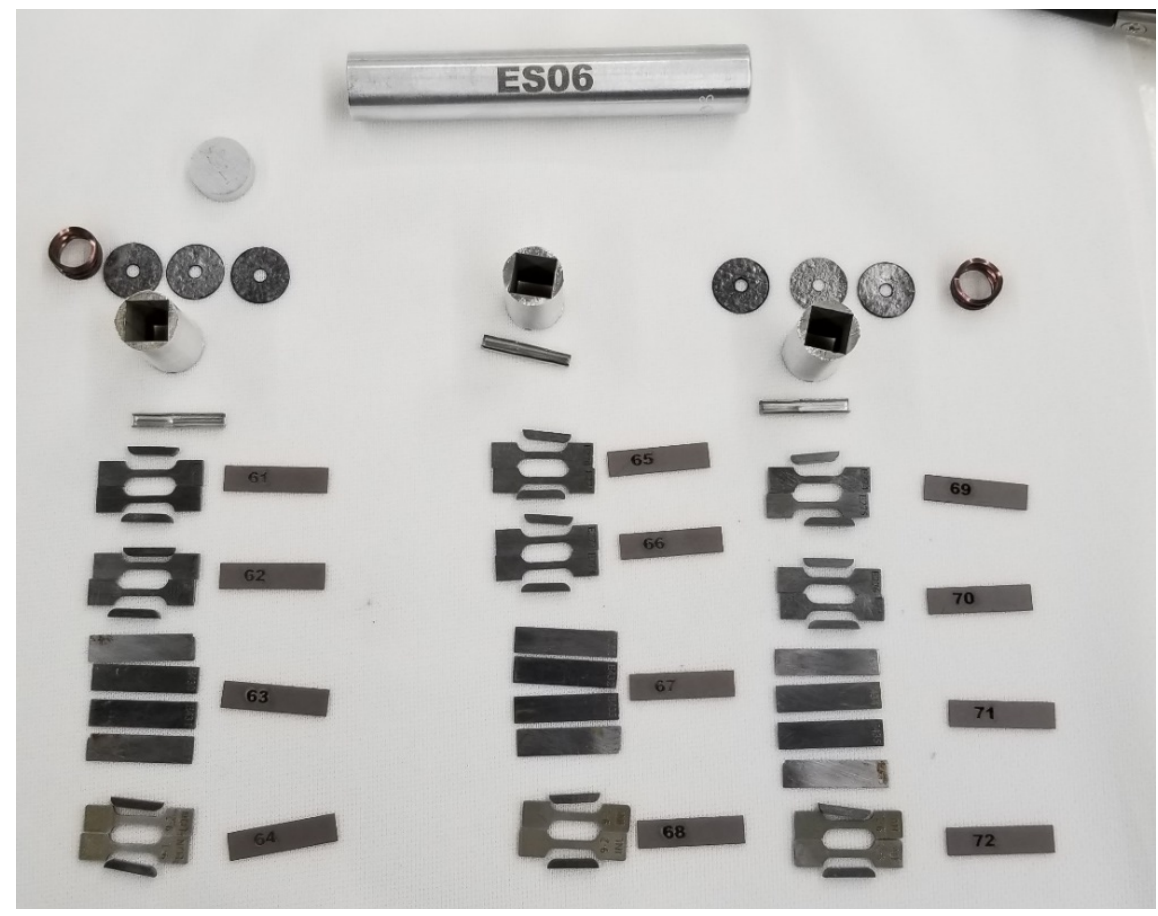

Fig. A14. Parts layout for rabbit ES06. 


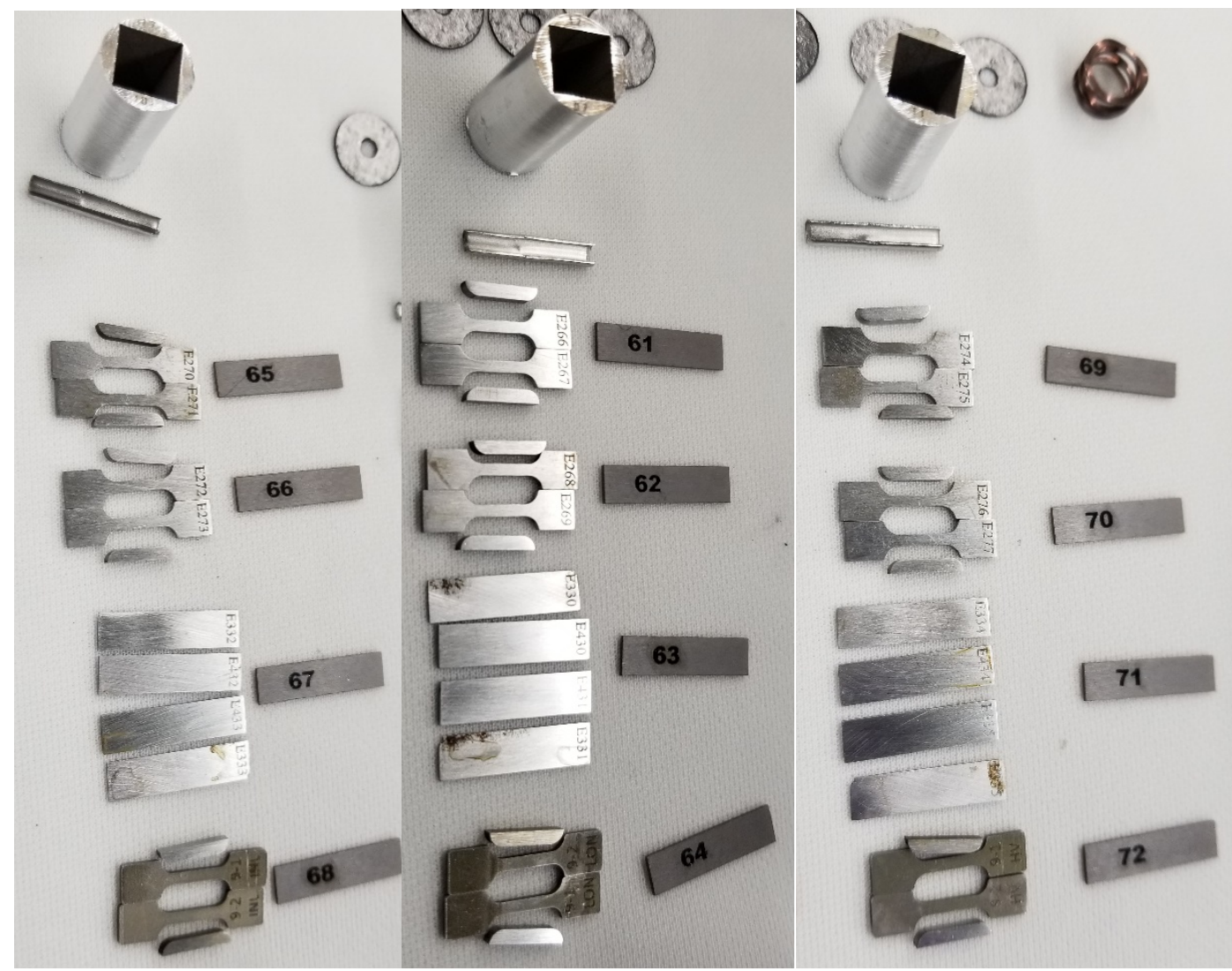

Fig. A15. Parts layout for the three holders of rabbit ES06.

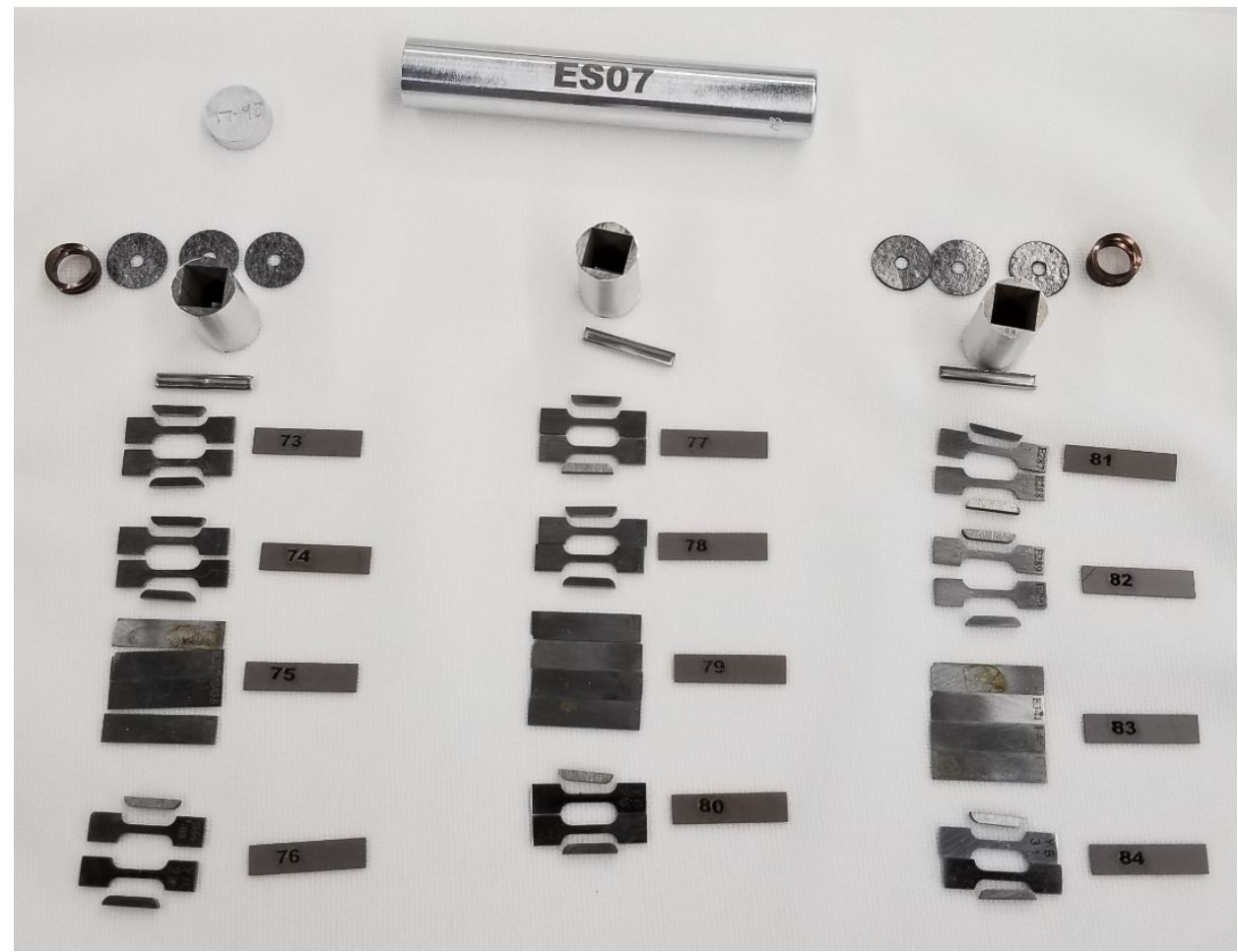

Fig. A16. Parts layout for rabbit ES07. 


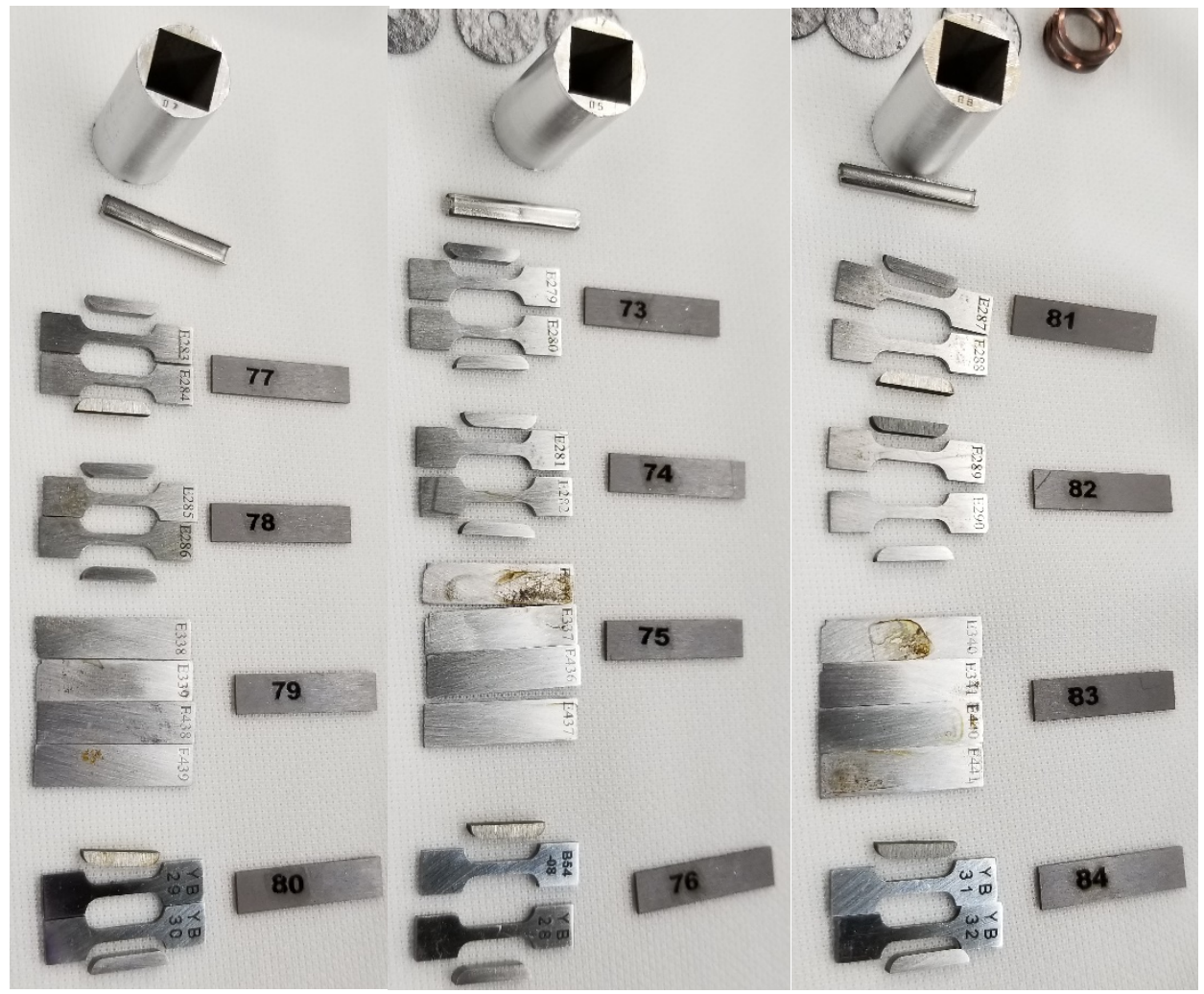

Fig. A17. Parts layout for the three holders of rabbit ES07. 


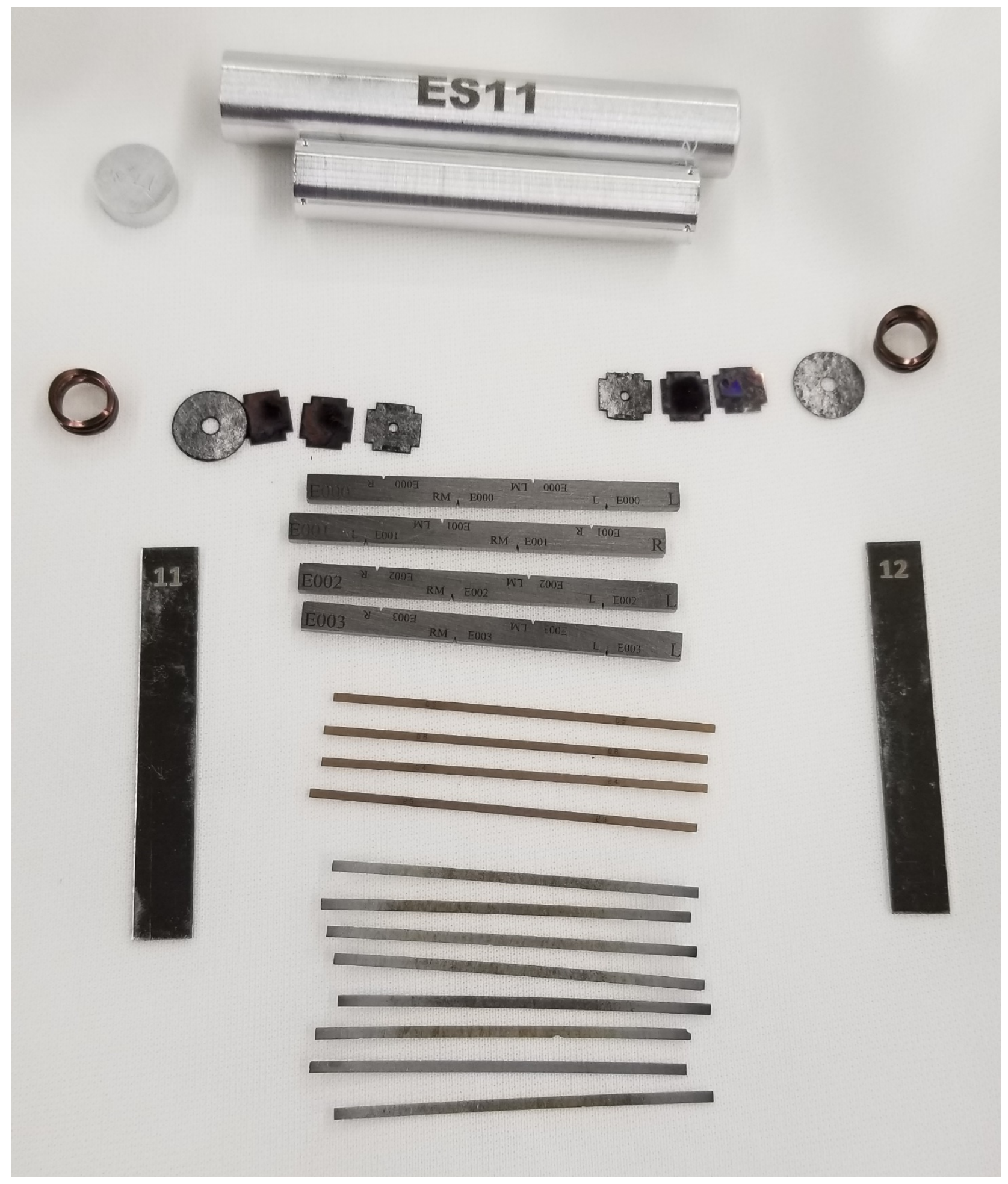

Fig. A18. Parts layout for rabbit ES11. 


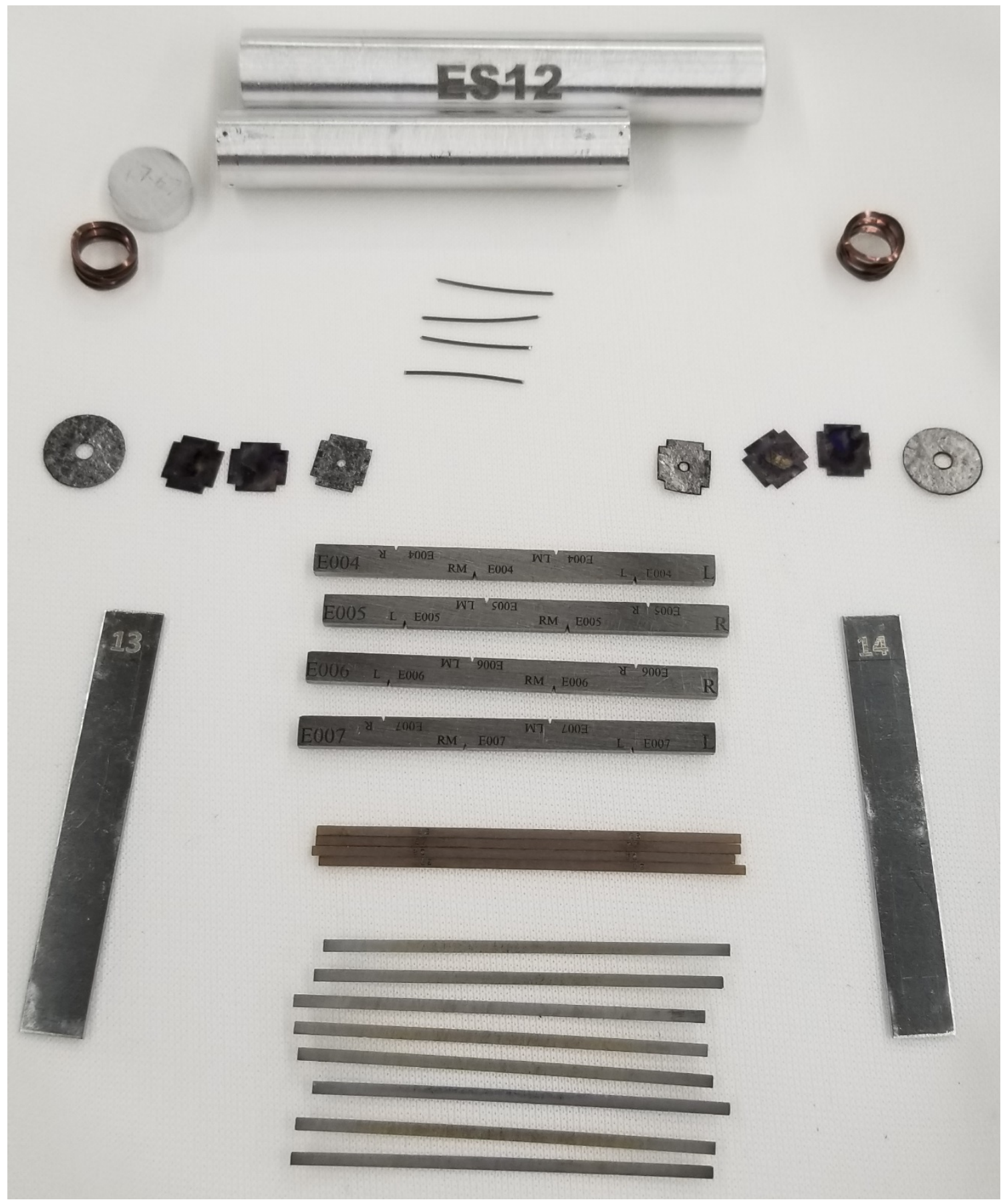

Fig. A19. Parts layout for rabbit ES12. 


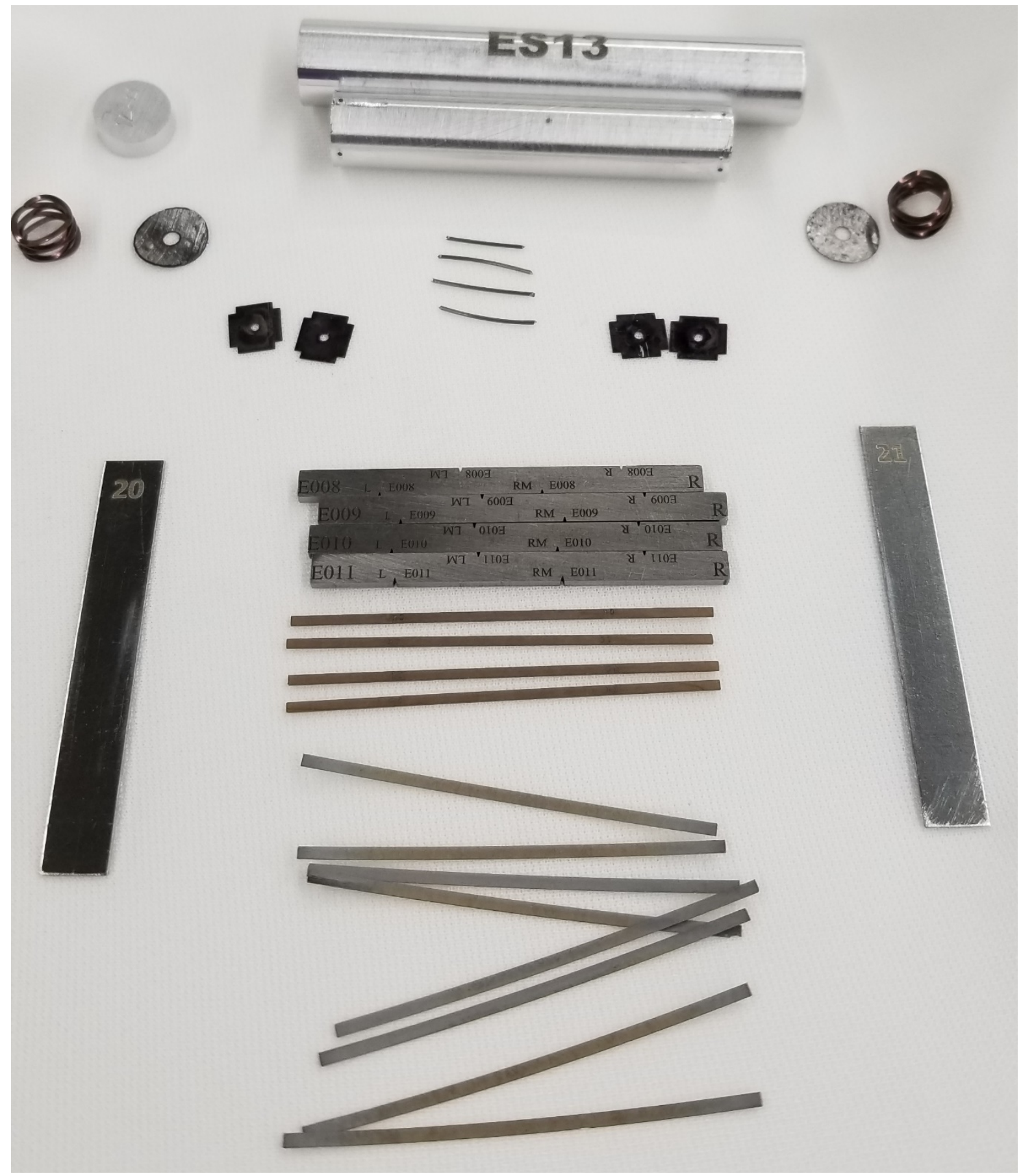

Fig. A20. Parts layout for rabbit ES13. 


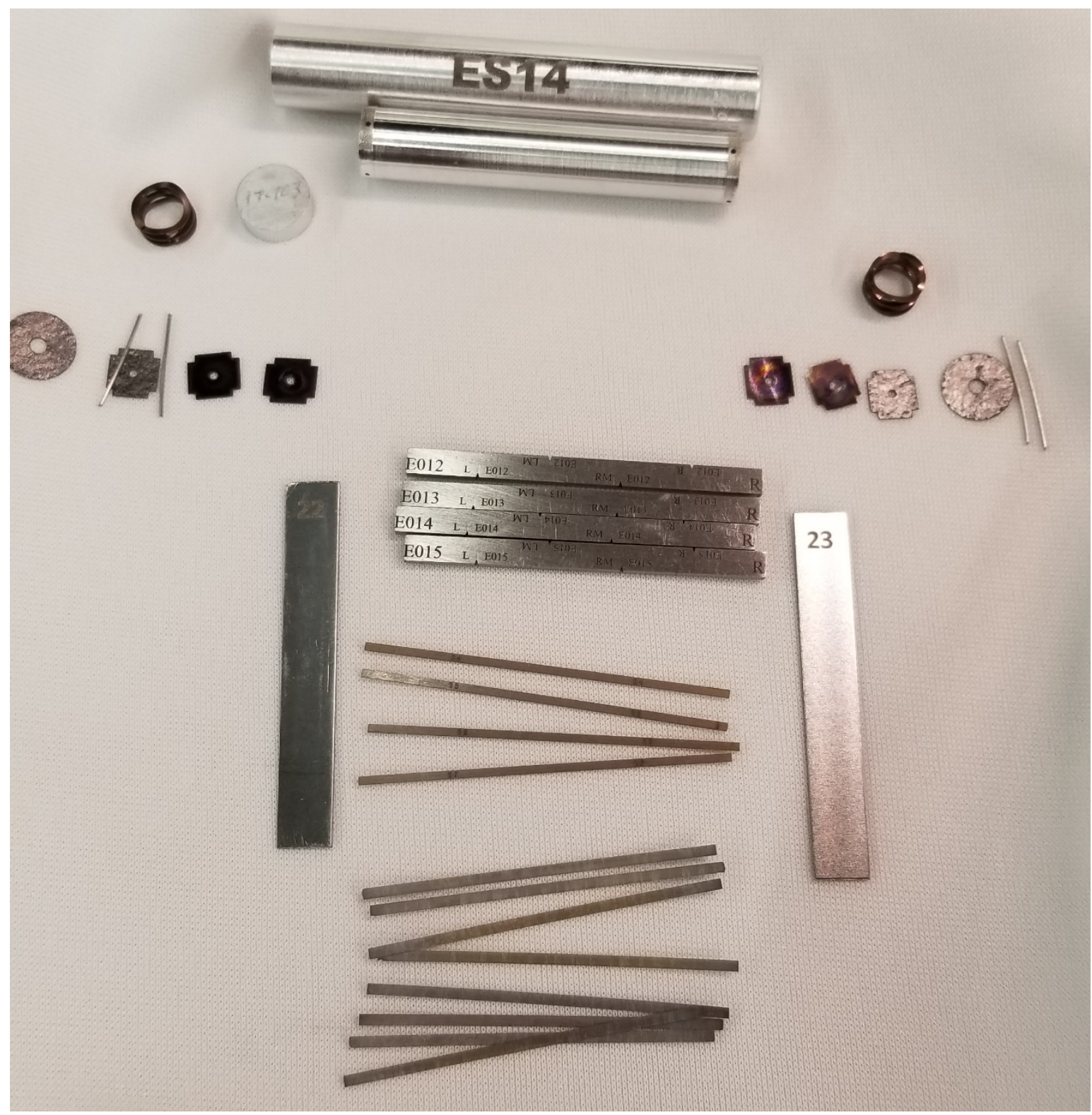

Fig. A21. Parts layout for rabbit ES14. 


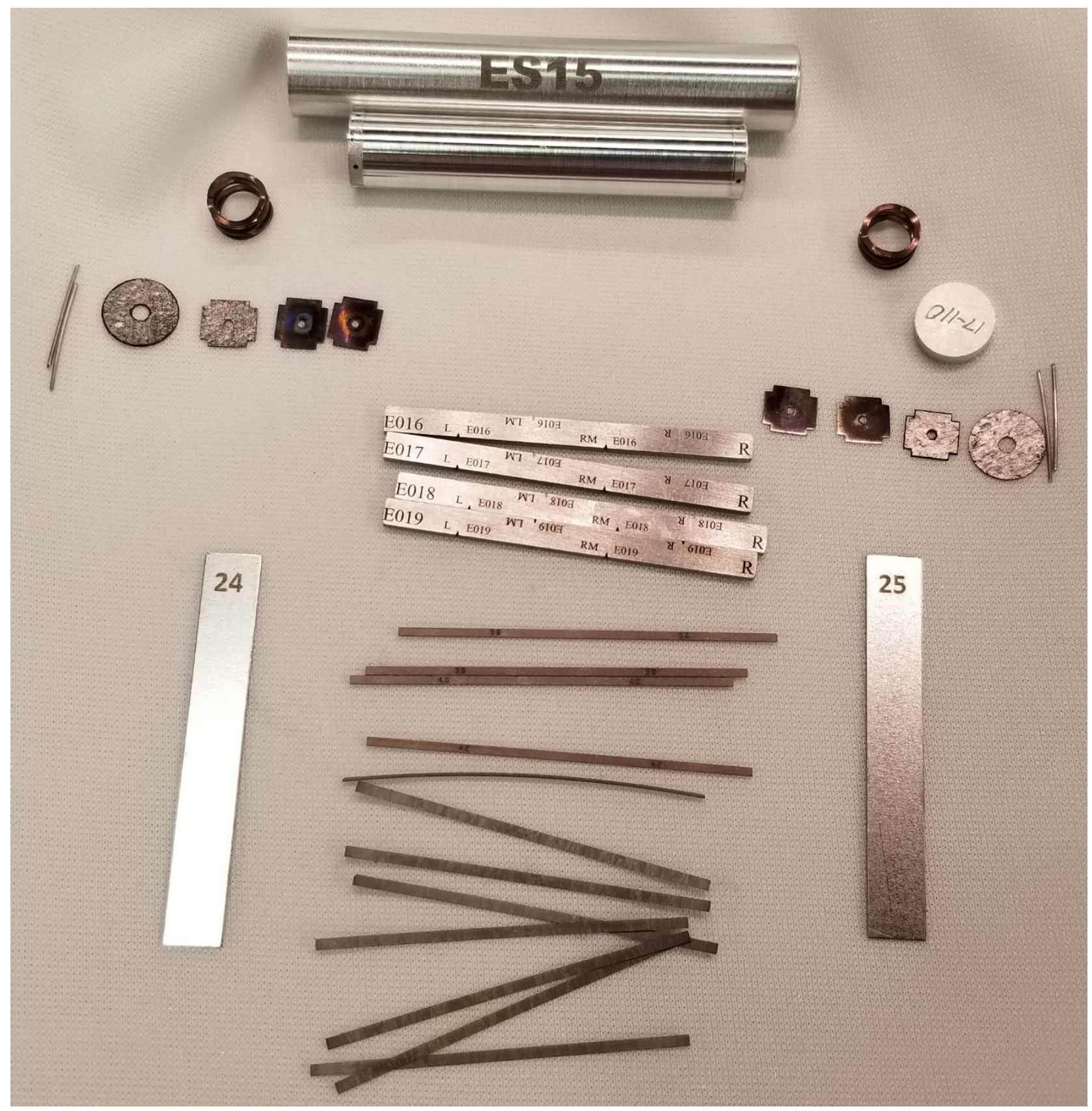

Fig. A22. Parts layout for rabbit ES15. 


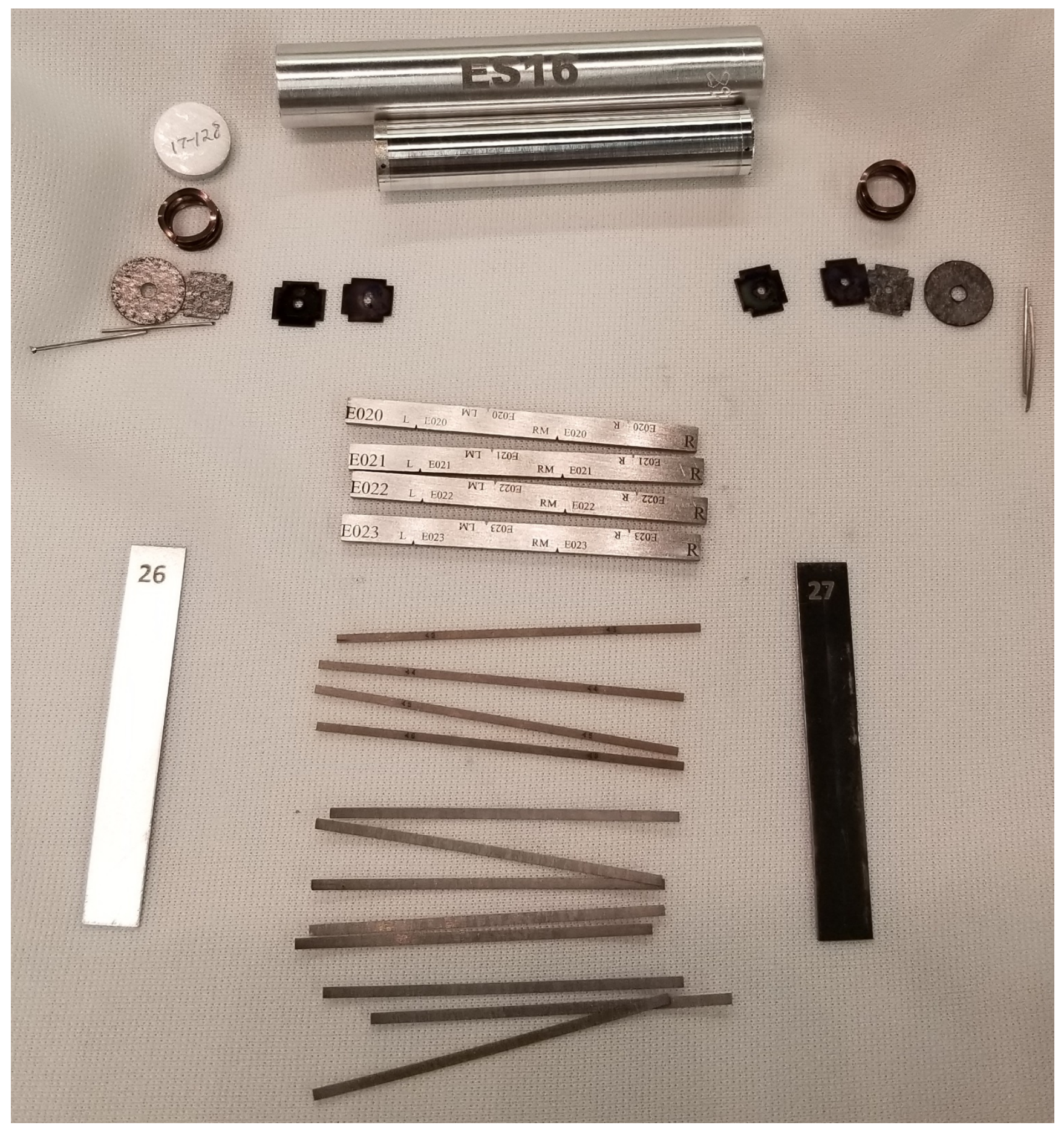

Fig. A23. Parts layout for rabbit ES16. 


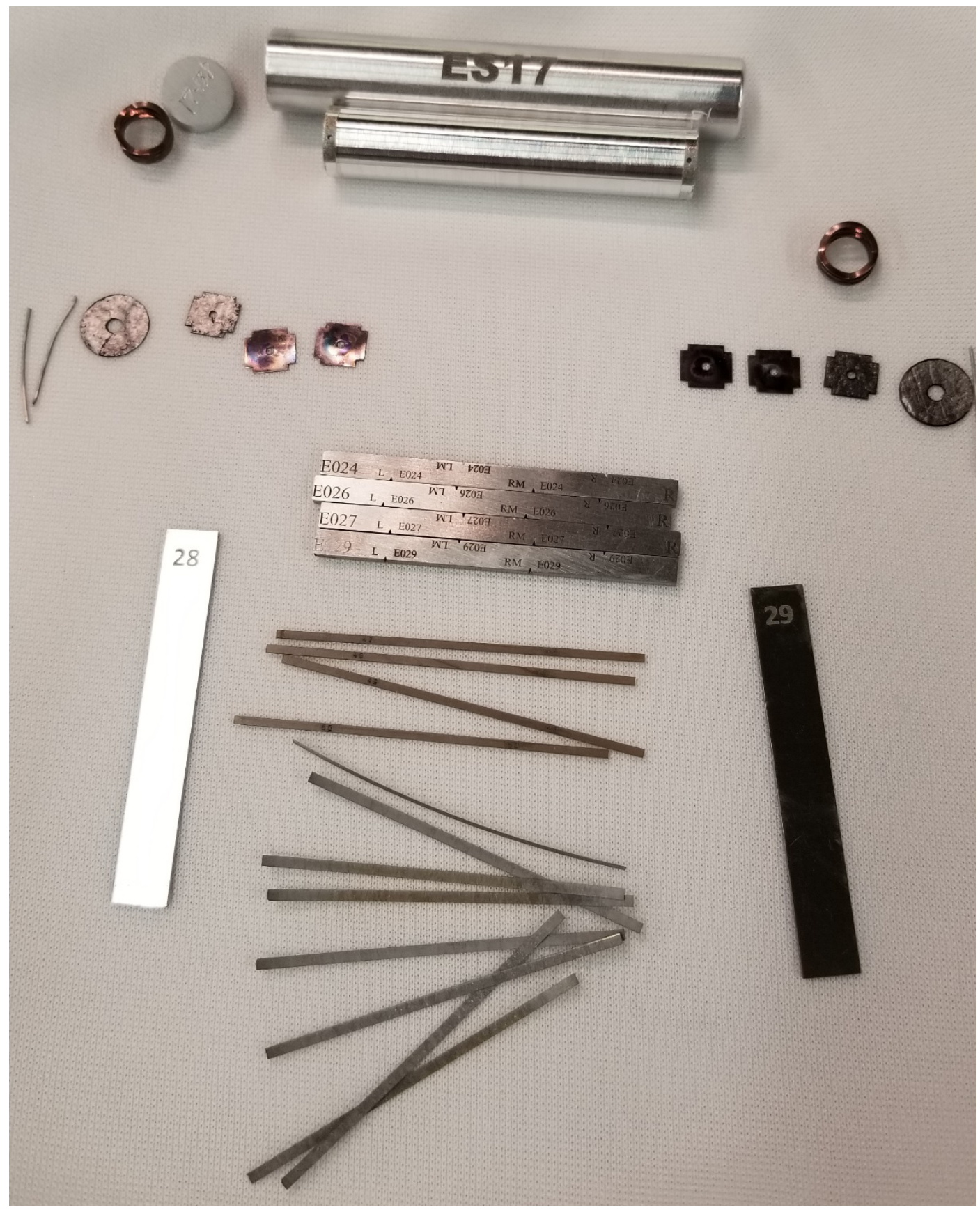

Fig. A24. Parts layout for rabbit ES17. 


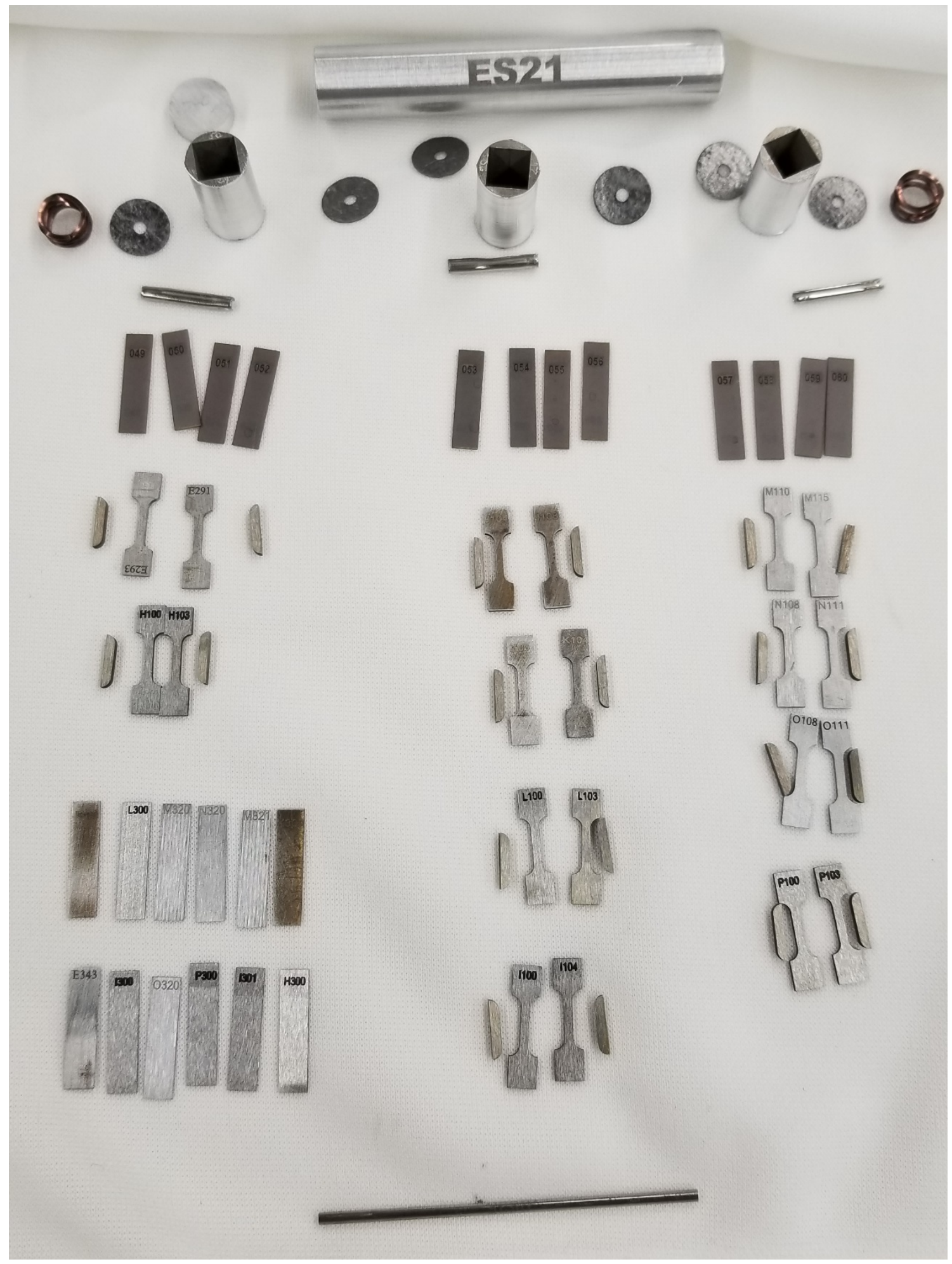

Fig. A25. Parts layout for rabbit ES21. 


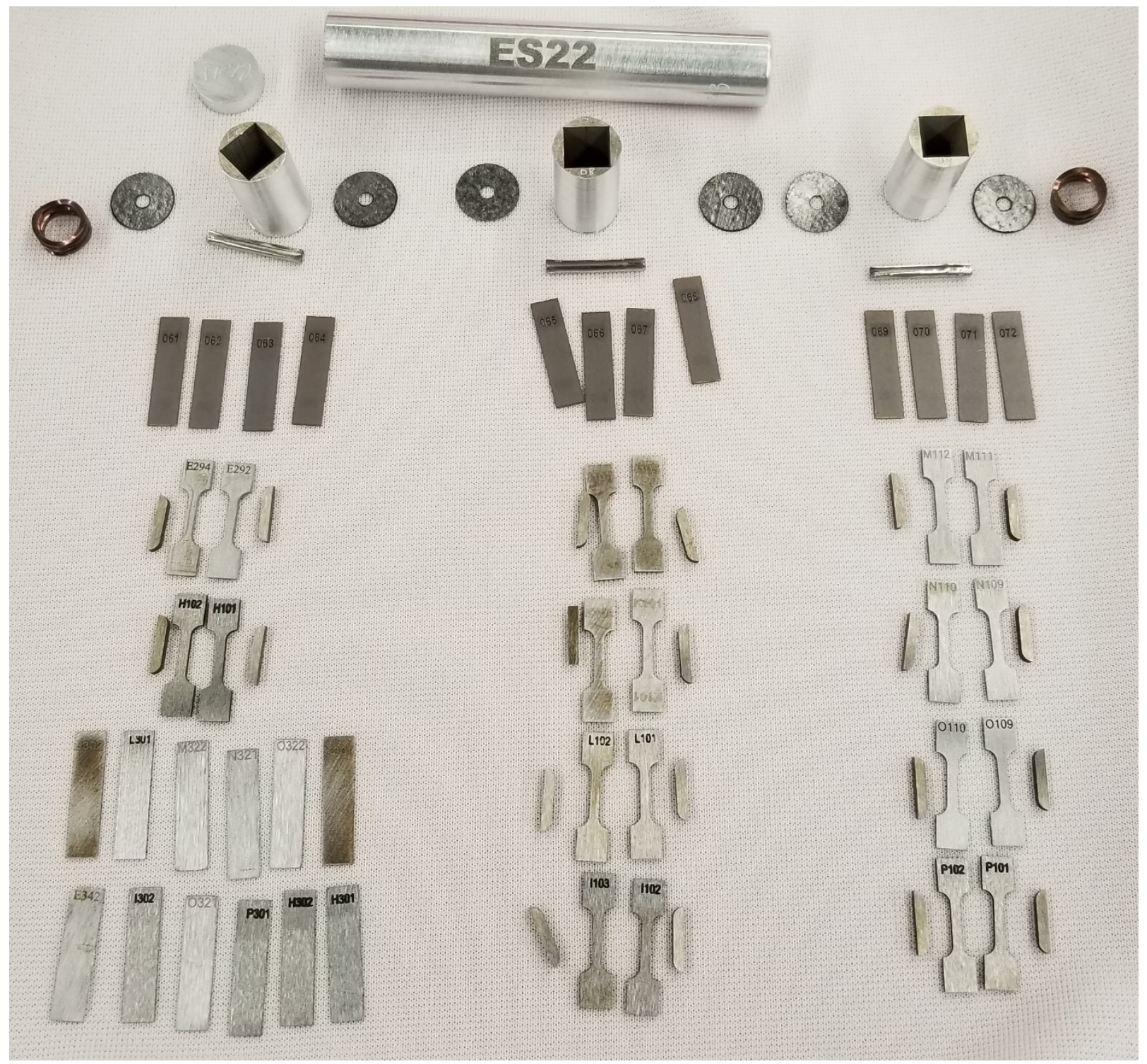

Fig. A26. Parts layout for rabbit ES22. 


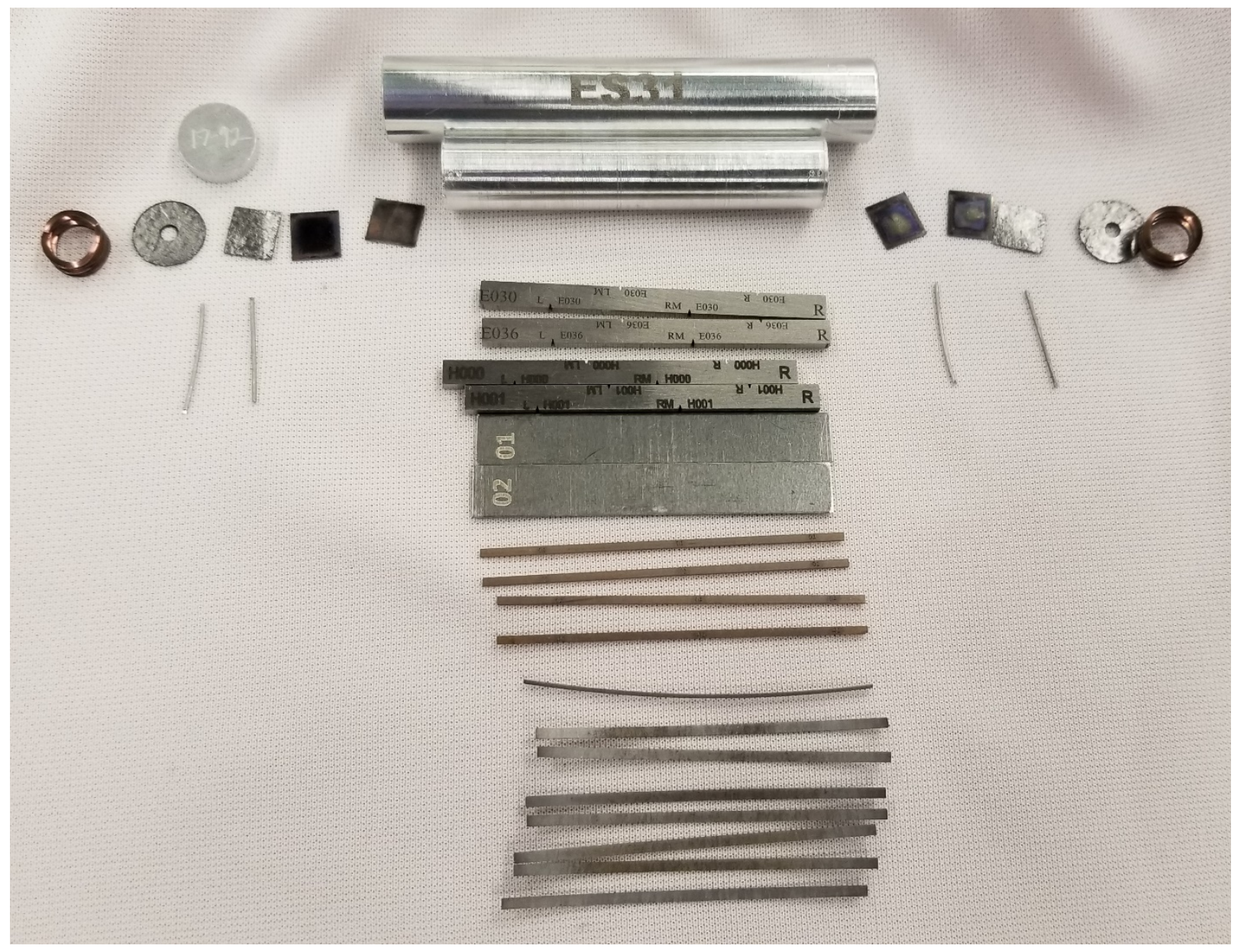

Fig. A27. Parts layout for rabbit ES31. 


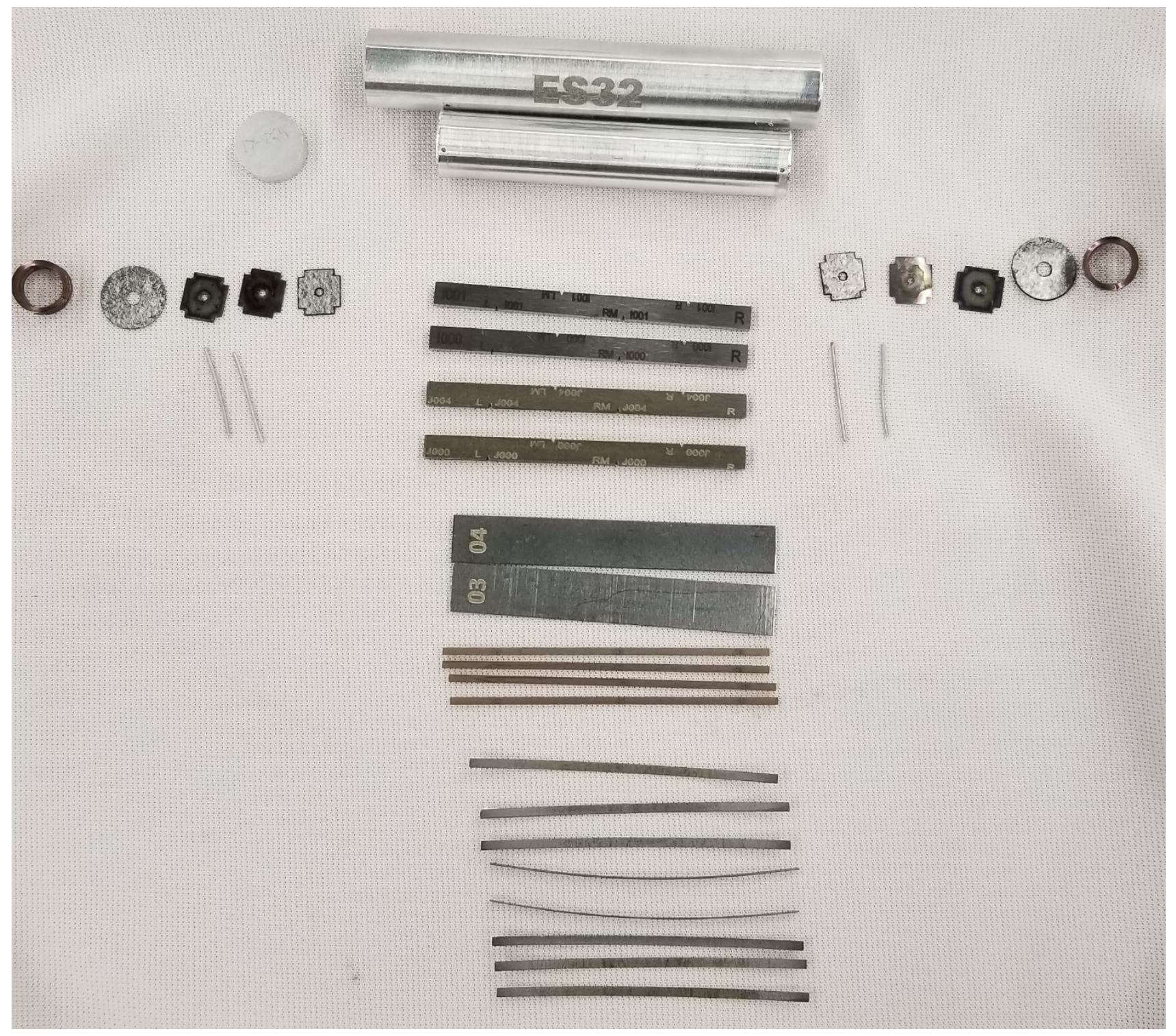

Fig. A28. Parts layout for rabbit ES32. 


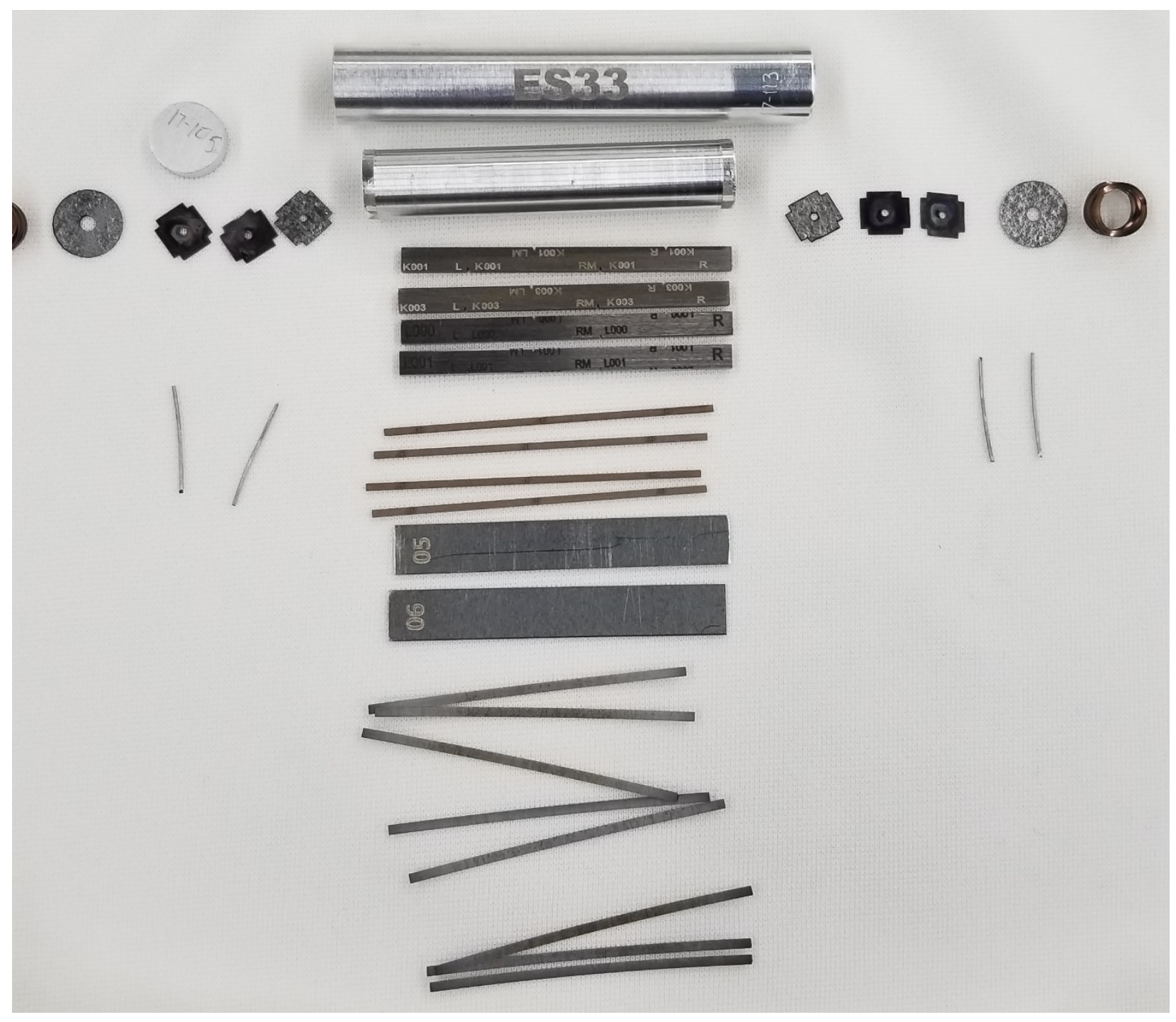

Fig. A29. Parts layout for rabbit ES33. 


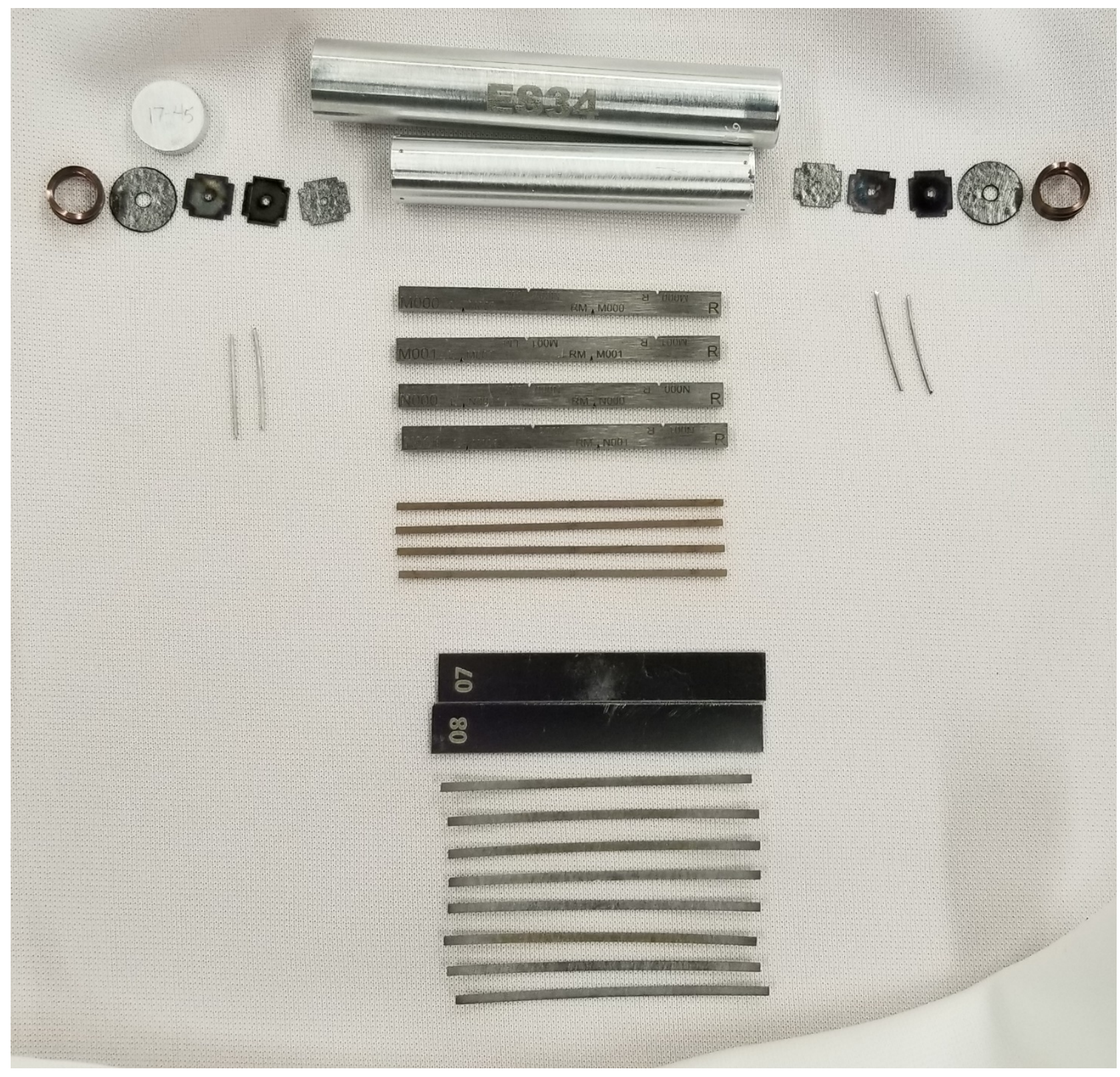

Fig. A30. Parts layout for rabbit ES34. 


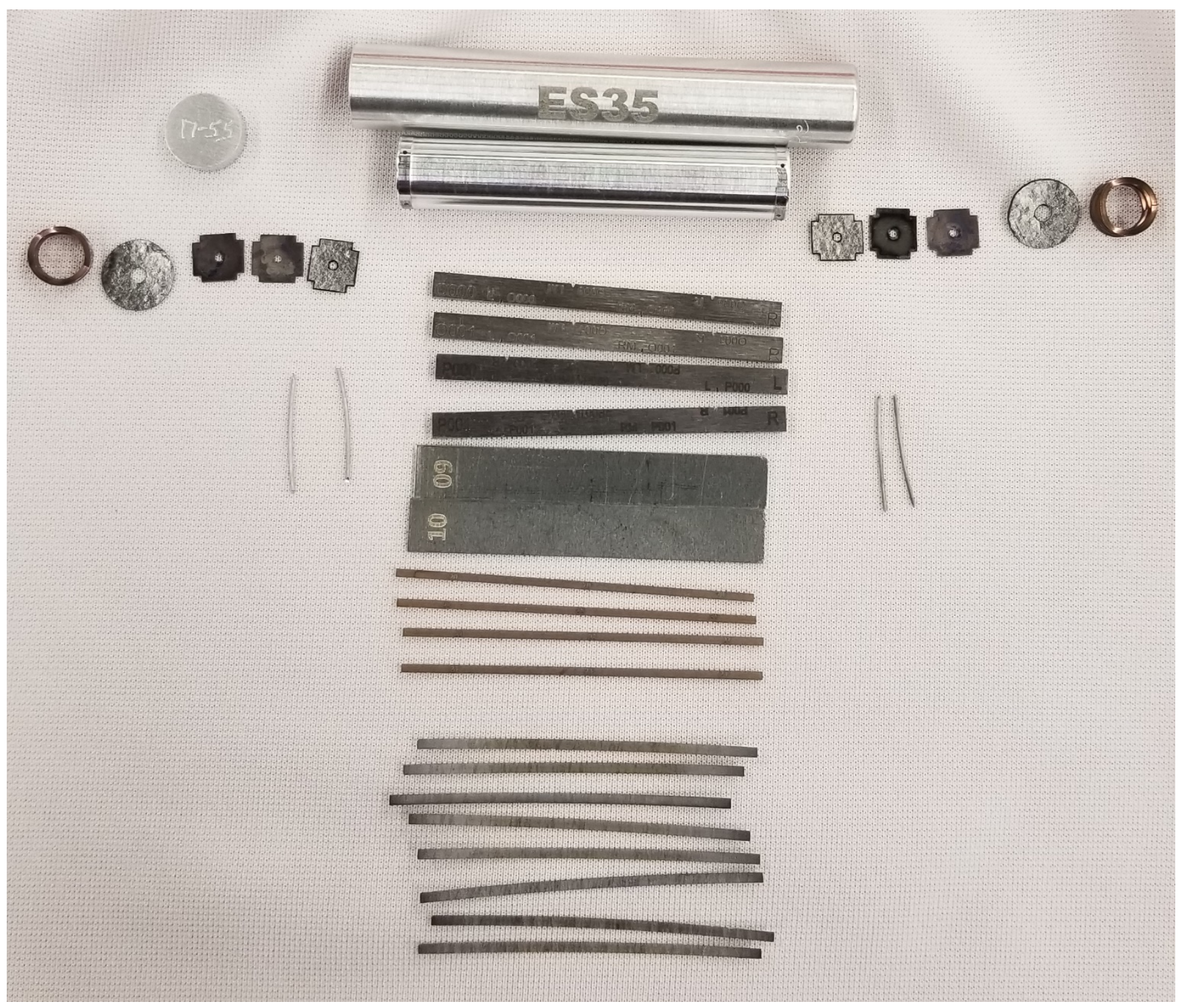

Fig. A31. Parts layout for rabbit ES35. 